\title{
Hydropower Case Study Collection: Innovative Low Head and Ecologically Improved Turbines, Hydropower in Existing Infrastructures, Hydropeaking Reduction, Digitalization and Governing Systems
}

\author{
Emanuele Quaranta ${ }^{1, *},+\left(\mathbb{C}\right.$, Manuel Bonjean ${ }^{2}$, Damiano Cuvato ${ }^{2}$, Christophe Nicolet ${ }^{3}$, \\ Matthieu Dreyer ${ }^{3}$, Anthony Gaspoz ${ }^{4}$, Samuel Rey-Mermet ${ }^{4}$, Bruno Boulicaut ${ }^{5}$, Luigi Pratalata ${ }^{6}$, \\ Marco Pinelli ${ }^{6}$, Giuseppe Tomaselli ${ }^{6}$, Paolo Pinamonti ${ }^{7}$, Raffael Pichler ${ }^{7}$, Paolo Turin ${ }^{8}$, \\ Daniele Turrin ${ }^{8}$, Jason Foust ${ }^{9}$, Bradly Trumbo ${ }^{10}$, Martin Ahmann ${ }^{10}$, Marc Modersitzki ${ }^{11}$, \\ Susy Kist ${ }^{12}{ }^{\circledR}$, Cecilia Mosca ${ }^{13}$, Carlo Malerba ${ }^{14}$, Ada Francesconi ${ }^{15}$, Ivan Casoli ${ }^{16}$, \\ Raffaele Ferrari ${ }^{17}$, Vittoria Stefani ${ }^{17}$, Marco Scibetta ${ }^{18}$, Lorenza Meucci ${ }^{18}$, Walter Gostner ${ }^{19}{ }^{10}$, \\ Riccardo Bergamin ${ }^{20}$, Francesco De Pretto ${ }^{20}$, Davide Turcato ${ }^{20}$, Vincent Kocher ${ }^{21}$, \\ Pierre Lefaucheux ${ }^{21}$, Abdelali Elmaataoui ${ }^{21}$, Mario Mariucci ${ }^{22}$, Prakriteesh Sarma ${ }^{23}$, \\ Geert Slachmuylders ${ }^{24}$, Riccardo Clementi ${ }^{25}$, Fabio Pasut ${ }^{25}$ and Nicola Bragato ${ }^{26}$ \\ 1 Politecnico di Torino, Politecnico di Torino, 10129 Torino, Italy \\ 2 Electromechanical Engineering Dept., Compagnia Valdostana delle Acque, 11024 Chatillon, Italy; \\ bonjean.manuel@cvaspa.it (M.B.); cuvato.damiano@cvaspa.it (D.C.) \\ 3 Power Vision Engineering Sàrl, CH-1025 Saint-Sulpice, Switzerland; \\ christophe.nicolet@powervision-eng.ch (C.N.); matthieu.dreyer@powervision-eng.ch (M.D.) \\ 4 HES-SO Valais, CH-1950 Sion, Switzerland; anthony.gaspoz@hevs.ch (A.G.); \\ samuel.rey-mermet@hevs.ch (S.R.-M.) \\ 5 Electricite d'Emosson SA, CH-1920 Martigny, Switzerland; bruno.boulicaut@emosson.ch \\ 6 Enel Green Power, 00198 Rome, Italy; luigi.pratalata@enel.com (L.P.); marco.pinelli@enel.com (M.P.); \\ giuseppe.tomaselli@enel.com (G.T.) \\ 7 Eisackwerk Srl, 39100 Bolzano, Italy; paolo.pinamonti@eisackwerk.it (P.P.); \\ raffael.pichler@eisackwerk.it (R.P.) \\ 8 Bioprogramm S.c., 35127 Padova, Italy; pturin@bioprogramm.it (P.T.); daniele.turrin@bioprogramm.it (D.T.) \\ 9 Voith Hydro, Inc., York, PA 17408, USA; jason.foust@voith.com \\ 10 US Army Corps of Engineers, Walla Walla District, Walla Walla, WA 99362, USA; \\ Bradly.A.Trumbo@usace.army.mil (B.T.); Martin.L.Ahmann@usace.army.mil (M.A.) \\ US Synthetic, Orem, UT 84058, USA; marc@ussynthetic.com \\ Ocean Renewable Power Company, Inc., Portland, ME 04011, USA; skist@orpc.co \\ Hydrodata S.p.A., 10123 Torino, Italy; mosca@hydrodata.it \\ 4 INTECNO Ingegneria e Tecnologia S.r.l., 10123 Torino, Italy; malerba@intecnoholding.com \\ 5 Consorzio di Bonifica Emilia Centrale, 42121 Reggio Emilia, Italy; afrancesconi@emiliacentrale.com \\ 6 Studio di Progettazione AISE Engineering, 42123 Reggio Emilia, Italy; info@aiseengineering.com \\ 17 Ingegneri Consulenti srl, 38121 Trento, Italy; raffaele.ferrari@ingegnericonsulenti.com (R.F.); \\ vittoria.stefani@ingegnericonsulenti.com (V.S.) \\ 18 Società Metropolitana Acque Torino (SMAT), 10152 Torino, Italy; marco.scibetta@smatorino.it (M.S.); \\ lorenza.meucci@smatorino.it (L.M.) \\ 19 Patscheider \& Partner Engineers Ltd., 39024 Mals (BZ), Italy; w.gostner@ipp.bz.it \\ 20 ZECO Hydropower S.r.l., 36030 Fara Vicentino, Italy; riccardo.bergamin@zeco.it (R.B.); \\ francesco.depretto@zeco.it (F.D.P.); davide.turcato@zeco.it (D.T.) \\ 21 Pytheas Technology, 13590 Meyreuil, France; vincent.kocher@pytheas-technology.com (V.K.); \\ pierre.lefaucheux@pytheas-technology.com (P.L.); abdelali.elmaataoui@pytheas-technology.com (A.E.) \\ 22 Teti srl, 06024 Gubbio, Italy; Mario.mariucci@gmail.com \\ 23 Turbulent NV, 3012 Wilsele, Belgium; prakriteesh.sarma@turbulent.be \\ 24 ATWEO BV, 3012 Wilsele, Belgium; geertslachmuylders@gmail.com
}


STE Energy S.r.l, 35141 Padova, Italy; r.clementi@ste-energy.com (R.C.); f.pasut@ste-energy.com (F.P.)

RNB Hydro, 30030 Pianiga, Italy; nicola.bragato@rnb-hydro.com

Correspondence: quarantaemanuele@yahoo.it or Emanuele.quaranta@ec.europa.eu

Current address: European Commission Joint Research Centre, 21027 Ispra, Italy.

Received: 31 July 2020; Accepted: 20 October 2020; Published: 26 October 2020

check for updates

\begin{abstract}
Hydropower remains a key renewable energy source in the pursuit of the decarbonization of the economy, although the relatively high potential impact of the hydro-morphological alterations it may cause poses significant concerns for aquatic ecosystems. In the last years, new technologies and practices have been increasingly adopted to minimize the impacts of hydropower plants, while improving efficiency and flexibility of energy generation. The overall effect of these innovations may be a more sustainable design and operation of hydropower, striking a better balance between the objectives of decarbonization and ecosystem protection. This contribution presents and discusses a few representative examples of hydropower installations from companies in Italy, France, Switzerland, Belgium and the USA, where solutions have been adopted in this direction. The case studies cover (1) ecologically improved and low head hydropower converters (Vortex turbine, Hydrostatic Pressure Machine, VLH and Girard-optimized turbines, hydrokinetic turbines), hydropeaking reduction (2) new control systems, governors and digitalization, (3) hydropower as a strategy for local sustainable development and (4) energy recovery in existing hydraulic infrastructures and aqueducts. It was found that better-governing systems can extend the life span of runners, for example avoiding the runner uplift during a trip. Digitalization can improve efficiency by $1.2 \%$. New sustainable practices and turbines with better ecological behavior can minimize environmental impacts, like the reduction of fish mortality, improvement of fish habitat availability, reduction of oil for lubrication purposes and generation of economic incomes for local development. The use of existing structures reduces the total installation cost: examples are the total saving of $277 € / \mathrm{kW}$ by reusing irrigation pipes and reservoirs, or the reduction of the investment period from 9 years to 6 years by turbining the environmental flow. Innovative low head hydropower converters can exhibit good ecological behavior, with reduced costs $(<5000 € / \mathrm{kW})$ especially when installed in existing weirs. Results are discussed, contextualized and generalized to provide engineering data and tools to support future realizations of similar case studies; normalized costs, efficiency improvement, best practices and new technologies are discussed.
\end{abstract}

Keywords: bearing; digitalization; fish; governing system; hydropeaking; hydropower; low head turbine; RHPM; VLH; Vortex; water distribution network

\title{
1. Introduction
}

The global installed hydropower capacity is more than $1300 \mathrm{GW}$. According to the Global Renewables Outlook 2020 of the International Renewable Energy Agency (IRENA), this figure will need to grow by around $60 \%$ by 2050 to deal with the increasing energy demand. While the reduction of the energy demand seems not to be feasible, due to the economic growth, the use of renewable energy and more efficient technologies can help to reach the decarbonization objectives, allowing us to limit the rise in global temperature in line with the 2015 Paris Accord. The hydropower growth would generate 600,000 skilled jobs over the coming decade and would require an estimated investment of US\$1.7 trillion [1]. In addition to energy generation and job opportunities, large hydropower ( $>10 \mathrm{MW}$ ) may bring in some circumstances additional benefits, including water management, improvement of leisure activities and stabilization of the electric grid [2], while small hydro can contribute to local sustainable development and market opportunities in remote areas. At the same time, hydropower installations may cause the interruption of the longitudinal continuity of the river, hydropeaking, upstream impoundment and other hydro-morphological alterations [3,4], 
with potentially far-reaching impacts. Some of these impacts can be minimized, e.g., providing a minimum ecological flow [5], but not at all. Therefore, although hydropower is a key renewable energy source for the reduction of carbon emissions and market development [5], it should be developed only after careful scrutiny of its sustainability [6].

Hydropower technology is quite affirmed. However, several improvements in flexibility and ecological behavior are under development, as well as more efficient governing and operating systems and practices. Emerging technologies are under development [7] aimed at increasing hydropower flexibility and its sustainability, and minimizing environmental impacts. Some state-of-the-art documents about hydropower technologies can be found in [7] on emerging technologies, in [8] for Francis turbine improvements, in [9] on pump-as-turbine (turbines that can work as pumps in pumped storage hydropower plants) and [10-13] for low head hydropower.

In particular, the new developments that hydropower must face, as well as the related trends, can be classified into the following categories:

- more flexible turbines to cope with the frequent grid instabilities and flow rate variability [8]: new regulation systems, e.g., the variable speed operation and new governors, is a major topic. Some of these aspects are here discussed with practical case studies (Sections 2-4, Sections 13 and 17);

- the need for pump-as-turbines working also in pumping mode for pumping-storage hydropower plants [9]; the transient operation and the shift from the turbine to pump mode is worth investigation;

- new low head hydropower converters to be used in irrigation canals [10,11]; some of these are here discussed, like the Very Low Head (VLH) turbine, the Hydrostatic Pressure Machine (HPM) and the Vortex turbine, with their strength and limitations. In particular, one of the most important matters in the low head field is the power take-off, since low head converters generally rotate quite slowly;

- more fish-friendly solutions (fish passages and turbines with good ecological behavior), and sustainable solutions to minimize environmental impacts [12]; fish passages alone do not completely avoid fish collision with turbines so that the ecological behavior of the turbines must be improved (Sections 6 and 16);

- hydro plant digitalization to prevent failures and to optimize the operation [7]; this is one of the most recent needs in the hydropower sector, Section 3;

- energy recovery from drinking water distribution networks, non-powered dams and from the ecological flow of large dams [13]; this solution avoids new interruptions of the longitudinal connectivity of rivers, and some study cases are here presented (Sections 9-13);

- sustenance of local communities and electrification of rural areas [14]; hydropower sometimes leads to social impacts, e.g., reallocation of residents or alteration of the environment, especially in the case of big hydro plants. On the other side, hydropower can generate social incomes and benefits, like electrification, new roads and services, and local development, as described in Section 7;

- new best practices, strategies and additional reservoirs to minimize hydropeaking, to improve fish habitat. New materials are also under development.

In this context, a collection of case studies is here presented, with real hydropower examples characterized by a novel technology or a novel approach to increase flexibility, improve the control/governing system, and reduce the impacts, for example by using more ecological technologies or by the exploitation of existing structures. The case studies here presented gives light and disseminate data and novel ideas that otherwise would have been available and well known only among the owners of such installations. Case study collections can be also found in $[15,16]$, where, however, the case studies are discussed in qualitative and descriptive terms rather than to find generalized engineering results. Therefore, the strength of this paper is that case studies here discussed contain novel considerations, engineering data and suggestions that can be used for similar case studies and the better design of 
hydropower plants, with practical suggestions and novel scientific results. For example, the performance and the cost of new hydropower converters are also discussed. The presented case studies are here summarized and then deeply described in proper sections. The last section is a discussion, where results are summarized and discussed, focusing also on similar technologies and limitations.

- Better control and governing of hydropower plants:

- Section 2. Novel speed governors for hydropower plants.

- Section 3. Digital twin for penstock fatigue monitoring.

- Section 4. Better management by digitalization software and de-sedimentation techniques.

- Fish-friendly solutions and reduction of environmental impacts:

- Section 5. An innovative solution to reduce the hydropeaking effect of hydroelectric power plants.

- Section 6. Biological Validation of Improved Direct Turbine Survival at Ice Harbor Lock and Dam.

- Section 7. Hydropower as a driving force for Alpine territorial development.

- Section 8. Diamond Bearings for hydrokinetic turbines.

- Recovery of existing structures for hydropower generation:

- Section 9. Hydroelectric development of an existing multiple-use reservoir.

- Section 10. Mini hydro on the environmental flow overflow structures.

- Section 11. Hydropower from the Susa Valley drinking water network.

- Section 12. Implementation of a multipurpose project on an irrigation plant by installation of an innovative counter pressure Pelton turbine.

- Section 13. Variable speed application for energy recovery hydropower in aqueducts.

- New low head hydropower converters:

- Section 14. Study of low-head hydrostatic pressure water wheels for harnessing hydropower of small streams.

- Section 15. The Mariucci turbine as the evolution of the Girard turbine.

- Section 16. Rehabilitation of a low-head gravitational vortex site with an improved vortex turbine.

- Section 17. The Very Low Head turbine in navigation locks.

\section{New Speed Governors for Hydropower Plants: Case Study of Hone 1 Power Plant}

The speed governor is fundamental for the functioning of hydroelectric units [17]. Initially, the speed governor brings the turbine and the alternator to the nominal speed. The Parallel Device sends specific commands to the speed governor to carry out the parallel operation between the hydroelectric group and the electric grid. During the electrical energy production process, the speed governor automatically adjusts the turbine power to the grid's power variations by monitoring the rotational speed $(N[\mathrm{rpm}])$ of the hydroelectric unit. When the machine switch is closed, the number of revolutions of the turbine is synchronous with the grid frequency. The automatic frequency/power regulation is required by TERNA (Italian operator of high voltage electricity grids) for groups with power not lower than $10 \mathrm{MW}$. Specifically, Annex A15 [18] prescribes the automatic frequency/power compensation requirements and methods that the speed governor must have. As a general rule, through a transfer function, each speed governor regulates the flow of water directed towards the turbine to keep the rpm of the turbine constant. 


\subsection{RDF12@ of CVA Overcomes the Hydrodynamic Limits}

In 2011 Compagnia Valdostana delle Acque (CVA), Chatillon, Italy, started to design its own software to control its hydroelectric plants, to make them more efficient. The RDF12@ speed governor, developed by CVA, uses the transfer function obtained in the $90 \mathrm{~s}$ by the ENEL research centre [19]. CVA owns 32 hydroelectric power plants (equipped with Pelton, Francis and Kaplan turbines) scattered throughout the Aosta Valley. CVA currently has a total power of $934.5 \mathrm{MW}$ which equates to approximately 3 billion kWh of energy produced per year. The RDF12@ speed governor allows unifying the control system to simplify the Asset Management, to reduce the variety of strategic replacements, to level out the technical procedures of the intervention and to reduce the costs for the maintenance of the automation software. Nowadays, more than $50 \%$ of the hydroelectric power produced in Aosta Valley is operated by RDF12@ speed governors. The RDT14@ voltage governor was also developed by CVA and here described. Anyway, before speaking about it, it is worth giving a summary of the context. Refer to [20] for further details.

In the RDF12@ frequency governor, the parameters of the transfer function (TF), are calculated through simple mathematical formulas that take into consideration the hydraulic phenomena characteristic of penstocks. Specifically, for Pelton and Kaplan turbines, the RDF12@ governor through TF calculates the opening of the spear or distributor and through another transfer function coordinates the opening of the deflector to the opening of the spear, or the opening of the runner blade to the opening of the distributor opening. The coordinated opening of spear and deflector, or distributor and runner blade, is called the conjugation curve. The conjugation curve is usually provided by the turbine manufacturer and can be obtained through specific mathematical formulas for Pelton and Kaplan turbines. In the RDF12@ governor, the innovative implementation of the conjugation curve was made around the set of 11 segments instead of a continuous mathematical formula, in order to obtain the advantage of being able to fast correct the percentage of openings between spear and deflector or between the distributor and runner blade.

For Pelton turbines, in case of load rejection, the innovative logic of the RDF12@ governor inhibits the conjugation between spear and deflector so that the deflector can operate at maximum servomotor speed in order to deflect the water jet (Figure 1). Thanks to the innovative conjugation inhibition logic, the transfer function can load other operating parameters, which confer greater response speed to the RDF12@ governor without compromising the hydraulic safety of the penstock.

Also, for Kaplan turbines, in the event of load rejection, the logic of the RDF12@ governor inhibits the conjugation between the distributor and the runner blade. The RDF12@ governor can manage up to three different gradients of distributor closing speeds. The choice of the right speed depends on the position of the distributor. The RDF12@ governor controls the distributor closing percentage in order to adopt the correct gradients of distributor closing speeds. Similarly, the RDF12@ governor manages the closing of the runner blade with two different gradients of runner blade closing speed.

These possibilities of the RDF12@ governor to adopt fast dynamics for TF and to be able to handle the closures of deflectors, spears, distributors and runner blades with different logics have allowed overcoming some limits imposed by the hydraulic phenomena of the penstocks and typical hydraulic transients. In fact, in the event of load rejection, it is possible to have faster recovery of the machine rpm and less abrupt hydraulic transients. 


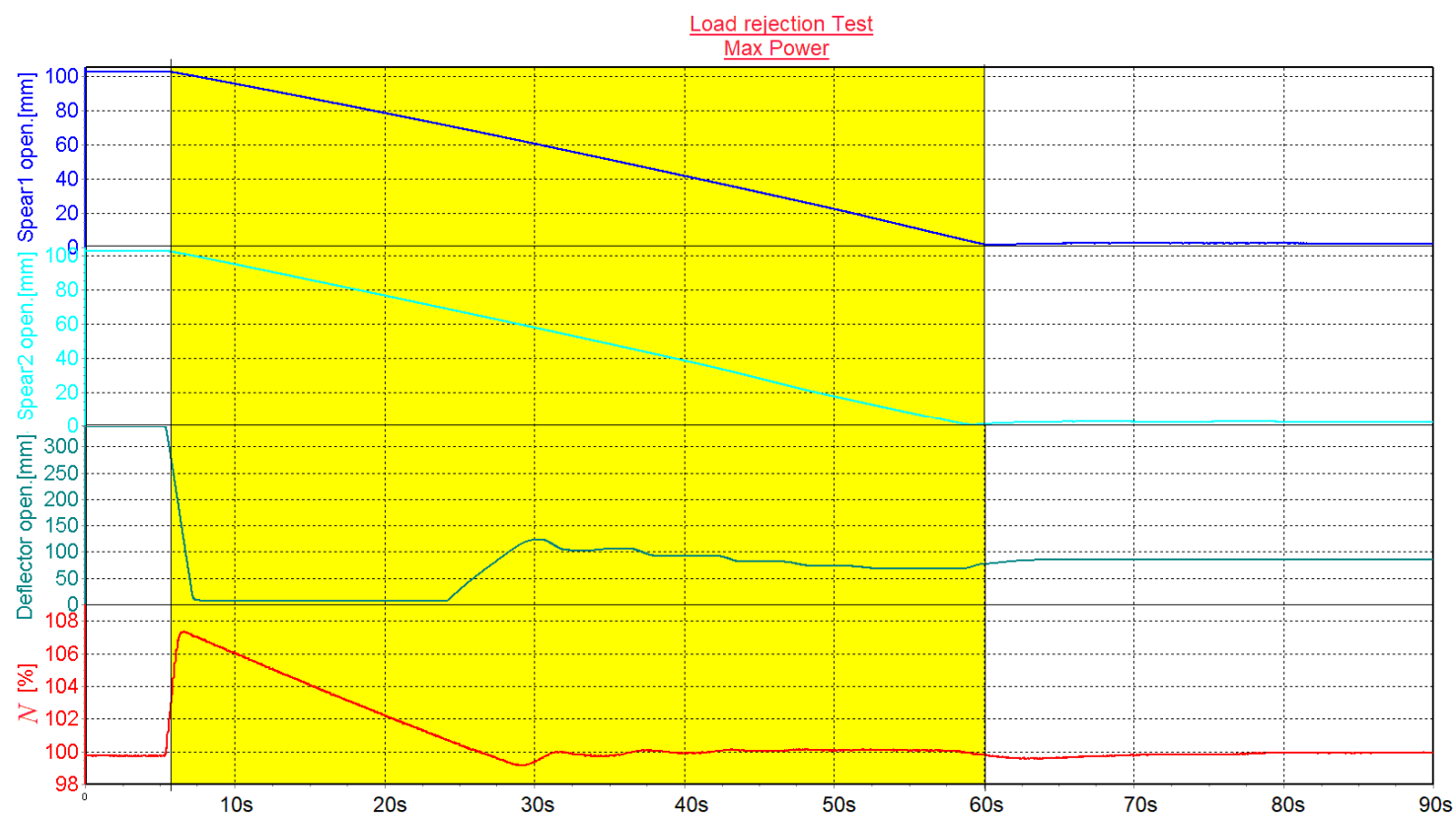

Figure 1. The figure shows the load rejection test on a two spears Pelton turbine with a maximum power of $20 \mathrm{MW}$. In the yellow area, thanks to the innovative logic of the RDF12@, the governor inhibits the nominal transfer function (TF) dynamic so the deflector can operate at maximum servomotor speed in order to deflect the water jet and subsequently reopen the deflector before the speed drops below the reference. In the white area, the logic of the RDF12(C governor re-establishes the nominal TF dynamics, so the opening deflector speed is the same as the spears speed.

\subsection{Hone 1 Power Plant}

In May 2018, CVA resolved a serious problem affecting the Kaplan turbine in operation at the Hone 1 power plant (Hone, Aosta Valley-Italy) [20], related to an abnormal behavior during the load rejection from high power $(>15 \mathrm{MW})$. The operating data that characterize the plant are gross head: $37.65 \mathrm{~m}$; nominal flow rate: $55 \mathrm{~m}^{3} / \mathrm{s}$; nominal power: 22.00 MVA (20 MW). For example, in the event of a trip in 1984, the behavior of the turbine was so abrupt to generate strong vibrations and the lifting of the turbine itself (Figure 2). To avoid this, the turbine has always been limited to operate at a power below 18.5 MW. To remedy the problem, the solution was to develop new closure laws of the distributor and the runner blade, based on numerical simulations (Figure 3). In particular, a bi-linear distributor closing law with a "knee" to better control the dynamic phenomena in the draft tube was used, in combination with a completely different procedure for the runner blades, which open instead of close. After several numerical tests, an optimized set of closing laws was found, and the real data measured in-situ are shown in Figure 4. The relevant diagrams, related to the 20 MW trip, indicated a far smoother dynamic behavior at the draft tube and a stronger reduction of rotor speed, which together avoided the lifting of $t$.

he rotor. In summary, the simulations showed that with a distributor closing law based on two-speed gradients in combination with the slow closing of the runner blade, an excellent behavior of the turbine would be obtained during the hydraulic transient. Thanks to the RDF12@ governor, it was possible to implement the new laws on closing the distributor and the runner blade because the RDF12@ governor foresees different operating dynamics (Figure 4). The overspeed is now significantly limited with respect to the max value previously experienced (123\% vs. $128.7 \%$, see Figure 2 ) and the lift of the rotor has been avoided. The RDF12@ governor has been installed in 12 power plants owned by CVA; the biggest controlled hydroelectric group is in place in Valpelline (power $75 \mathrm{MVA}$ ), the smallest one is in Nus (power 9 MVA). Refer to [20] for more details. 


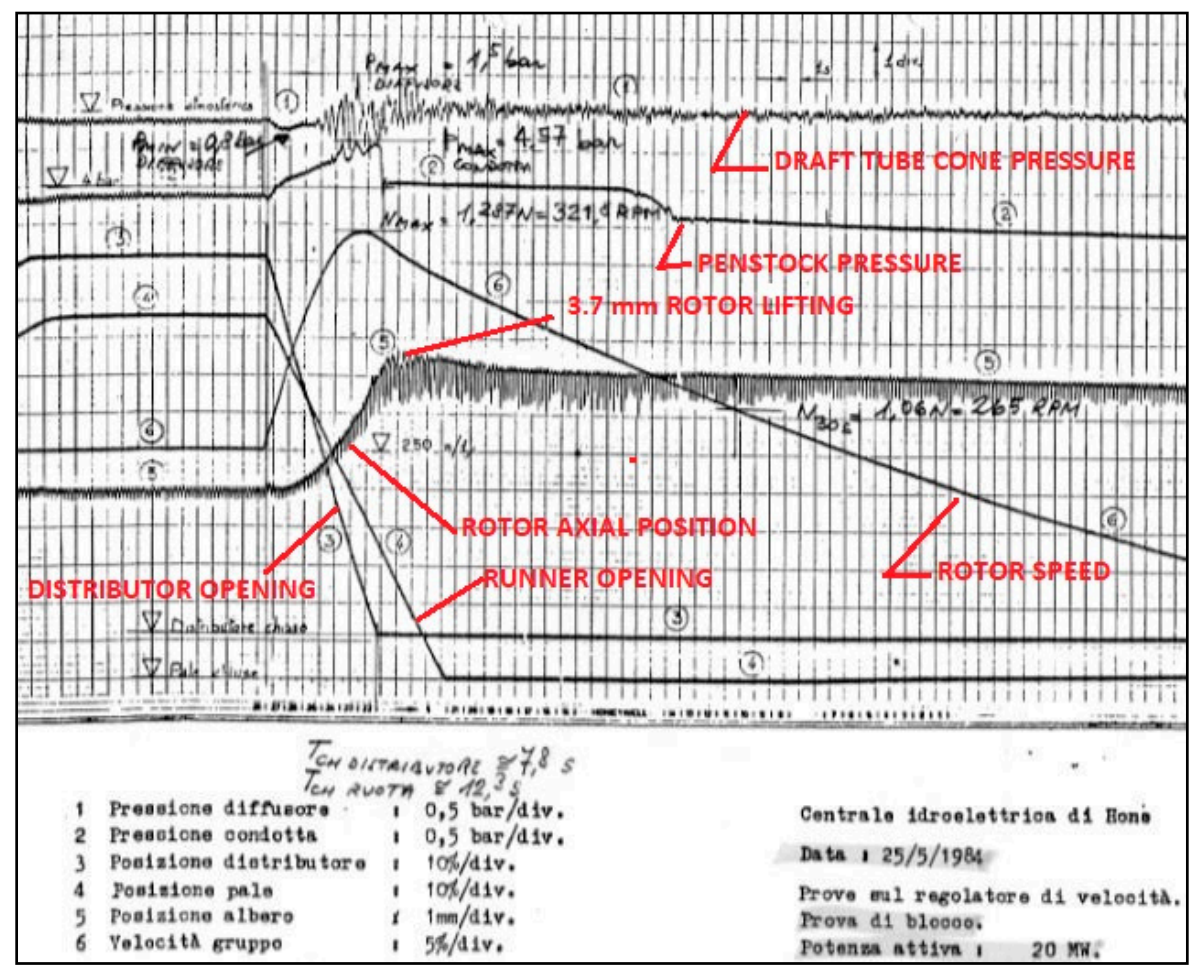

Figure 2. This recording shows the behavior of the Hone1 turbine during the hydraulic transients. The closing laws of the distributor and the blade runner are not optimized for the construction characteristics of the turbine. The maximum lift is recorded near the complete closure of the distributor and the runner blade.
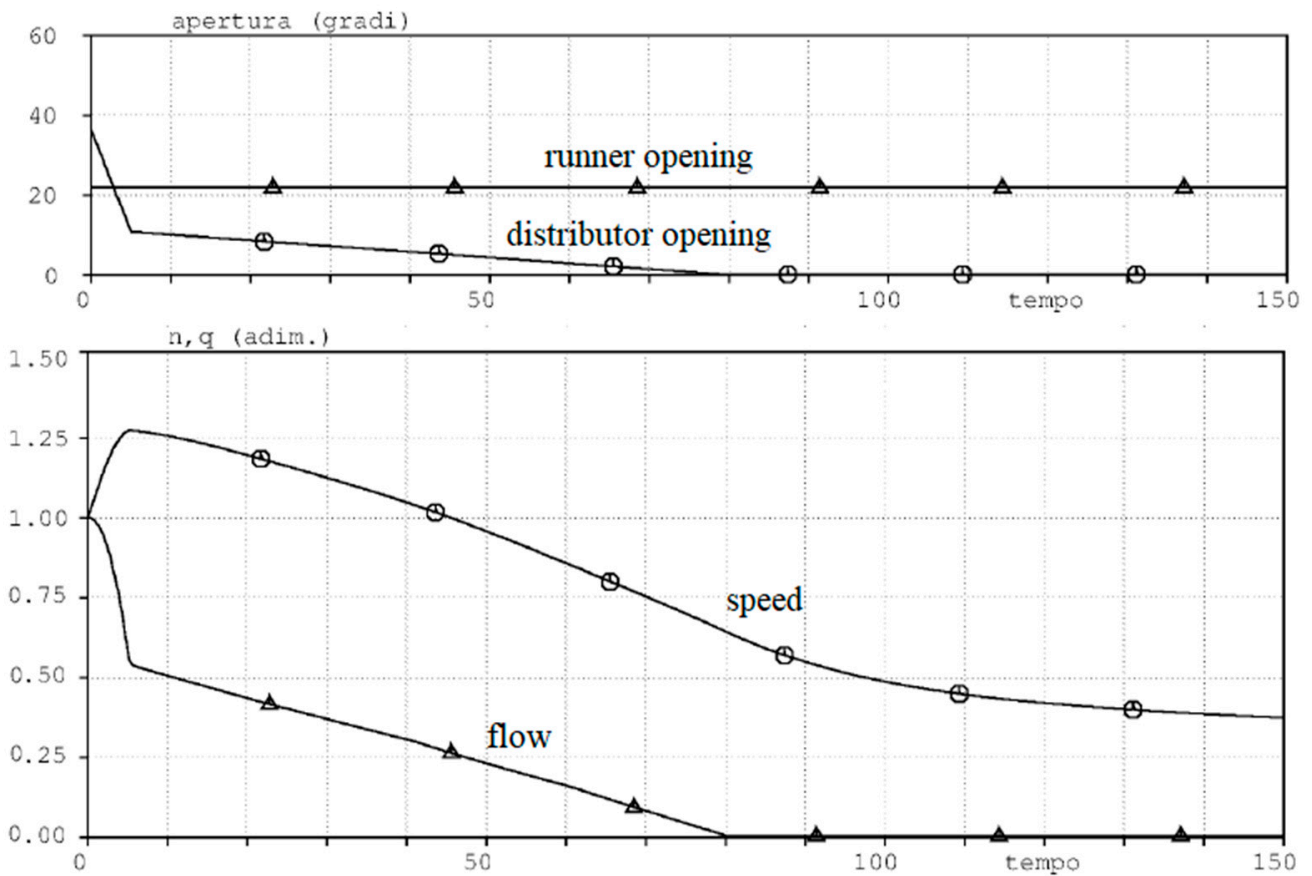

Figure 3. The guidelines for the definition of the new laws from numerical simulations [4]. Openings (top figure) and normalized rotational speed and flow rate. 


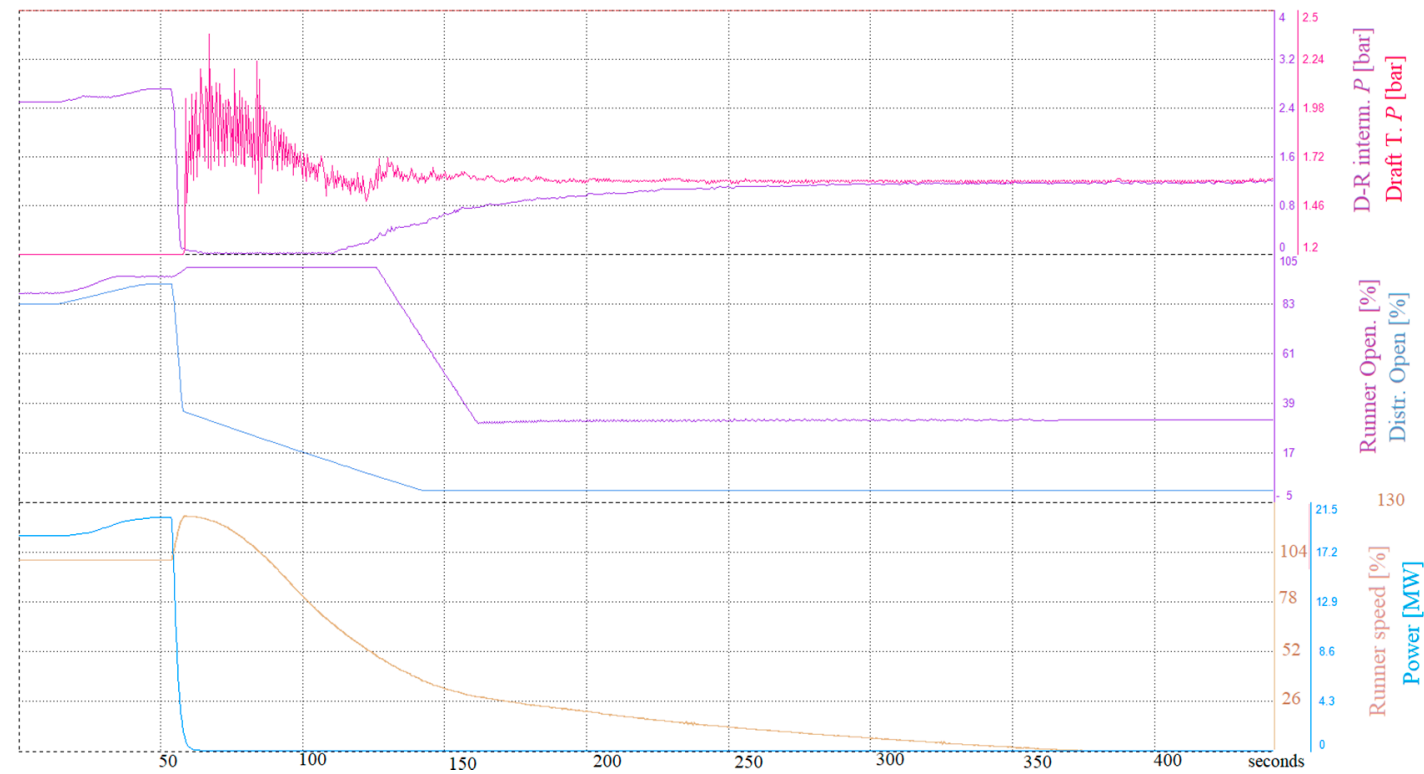

Figure 4. The above test diagram shows a $20 \mathrm{MW}$ trip versus the time (seconds). $P$ is the pressure. $D-R$ interm. is the space between the distributor and runner blades. Draft $T$. is the draft tube cone. On-site measurements.

\subsection{RDT14@ Voltage Governor}

The acquired know-how gave the possibility to develop the RDT14@ voltage governor. Unlike the RDF12@, the RDT14@ governor consists of a control part called RDT14-CU and a power part called RDT14-PU. The control part has two pre-set operating dynamics, the first for the no-load operation of the alternator and the second for the operation of the alternator closed on the electrical network. The first dynamic is used to align the amplitude of the alternator voltage with that of the electrical network. For example, when a $2 \%$ of voltage step is imposed, the response time is $T=224 \mathrm{~ms}$ (Figure 5), where $T$ is the first order time obtained from the intersection between the line at $102 \%$ voltage and the inclined line passing through the first steeper linear trend of the voltage increase. The second dynamic uses parameters calculated on the characteristics of the alternator to have a reactive and stable control system (Figure 6). The response time is $232 \mathrm{~ms}$, although the governor cannot bring the alternator voltage to the increase of $2 \%$ tension because the machine switch is closed on the electrical network. The RDT14@ governor has been installed in seven power plants owned by CVA; the biggest controlled hydroelectric group is in place in Valpelline (power 75 MVA), the smallest one is in Montjovet (power 28 MVA).

Obviously, regardless of the dynamics, the RDT14@ governor guarantees the operation of the alternator in accordance with the limits of the alternator capability. In addition, a current control mode to be injected into the rotor has been implemented on the RDT14-CU. In fact, during the commissioning of the RDT14@, or in order to carry out tests on the alternator, the mode of control of the current injected on the rotor facilitates the work of the technicians as it is simpler and safer to gradually increase the voltage of the alternator by controlling the rotor current. The RDT14@ power section is built to CVA specifications by an AC/DC converter manufacturer.

In conclusion, the way taken by CVA has saved time and money by improving the performance of its hydroelectric groups. 


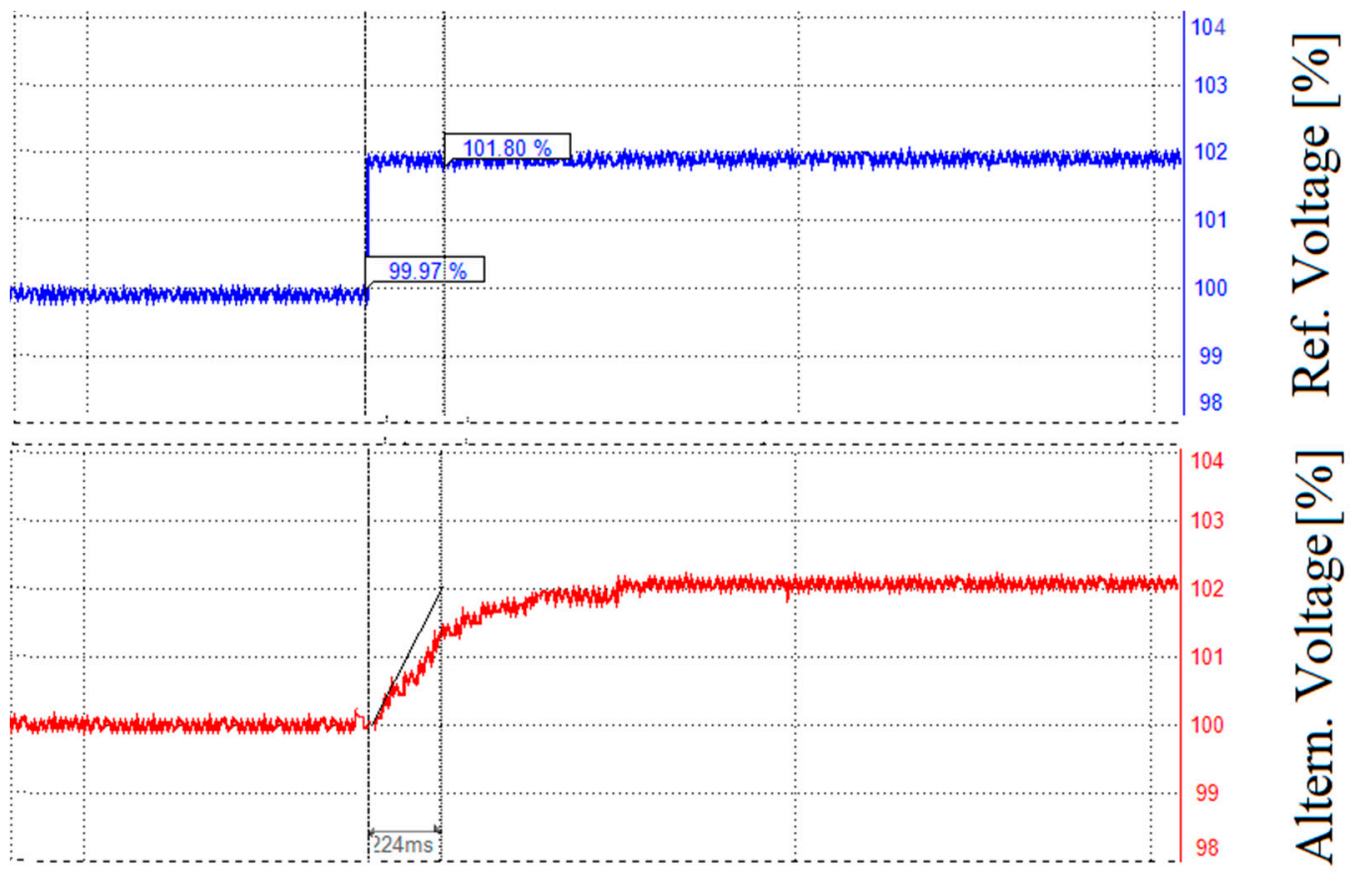

Figure 5. In this specific case, the RDT14@ governor is connected to an alternator of $16 \mathrm{MVA}, \cos \varphi 0.85$ and a nominal voltage of $5 \mathrm{KV}$. In the $2 \%$ amplitude of square pulse test on the voltage reference, the first-order response time is approximately $T_{1}=224 \mathrm{~ms}$. The vertical grid is every $1 \mathrm{~s}$.

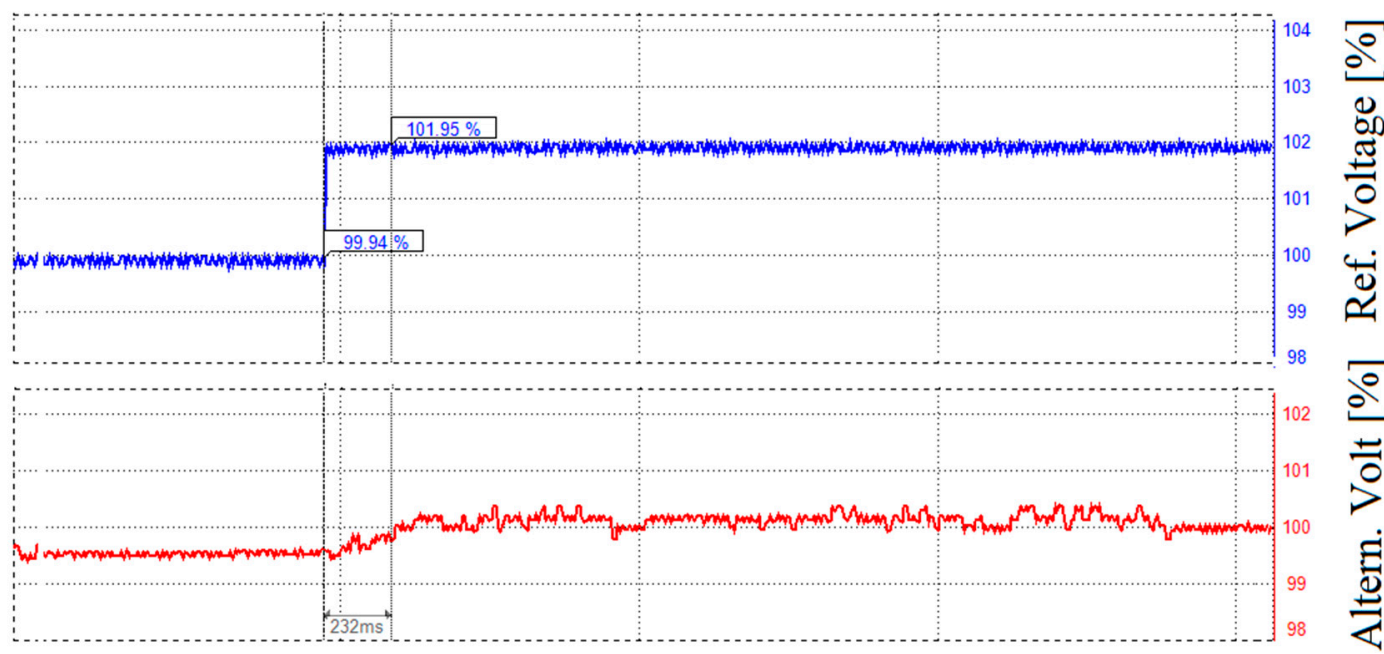

Figure 6. When the alternator is closed on the network, the RDT14@ governor adopts the second dynamic which considers the characteristics of the network. In a similar way to Figure 5 , in this $2 \%$ amplitude of the square pulse test, a response time of about $232 \mathrm{~ms}$ is obtained. The vertical grid is every $1 \mathrm{~s}$.

\section{Digital Twin for Penstock Fatigue Monitoring, Hydro-Clone Real-Time Simulation Monitoring System}

In Switzerland, most of the hydroelectric power plants were installed between 1950 and 1970. These power plants contribute considerably to the stability of the power grid through their operational flexibility and their ability to provide ancillary services. These services require frequent start-up and stop sequences, as well as continuous power variations leading to transient pressures in the conduits. Due to recent developments in the electricity market, existing hydroelectric power plants are subject to new operating conditions and sequences that were not foreseen at the design phase. 
This significant increase in load variations aggravates fatigue problems by stressing the penstock faster than expected [21-23]. Although load spectra are the fundamental element of any fatigue evaluation procedure, it is often difficult, if not completely impossible, to determine them accurately. In this case study, it is presented how the implementation of a digital twin of the power plant, namely the Hydro-Clone ${ }^{\circledR}$ real-time simulation monitoring system, can be used to fill this gap. By replicating the hydraulic transients of the power plant, the Hydro-Clone system allows monitoring in real-time the pressure variations throughout the waterways [24]. This capability is used to implement a fatigue module in Hydro-Clone by monitoring the fatigue level of the penstock, based on the damage accumulated during past and future operations. To validate this approach, the stress related to the pressure variation is measured in situ by the installation of strain gauges on the penstock of the 200 MW La Bâtiaz hydroelectric power plant, owned by Electricité d'Emosson SA, and compared to the simulated values. This approach reveals the significant impact of the provision of ancillary services on the fatigue wear of the penstock. This section presents the FEM analysis of the penstock of the La Bâtiaz power plant.

\subsection{Hydro-Clone}

Hydro-Clone is an innovative Real-Time Simulation Monitoring System (RTSM). It is a digital model of the hydropower plant (HPP) capable of reproducing in real-time any dynamic behavior of the plant based on the boundary conditions measured in situ (thus it is called digital twin [25]). Hydro Clone allows continuous diagnosis of the health of an HPP by digital cloning of the main hydraulic and electrical components of the plant using SIMSEN software [26]. The Hydro-Clone general concept is illustrated in Figure 7. The system handles the tasks of real-time acquisition and transfer of boundary conditions and measured quantities to the SIMSEN model, as well as data processing and diagnosis of the plant health status. A custom-built archival storage system and an associated database allow for the display and analysis of previous results. The analysis and comparison of simulated and measured quantities allow the health status and behavior of all essential system components to be assessed at any time and to estimate the non-measured/non-measurable quantities in the entire system [27]. The Hydro-Clone system was installed in 2014 at the La Bâtiaz power plant and has been in continuous operation for more than five years.

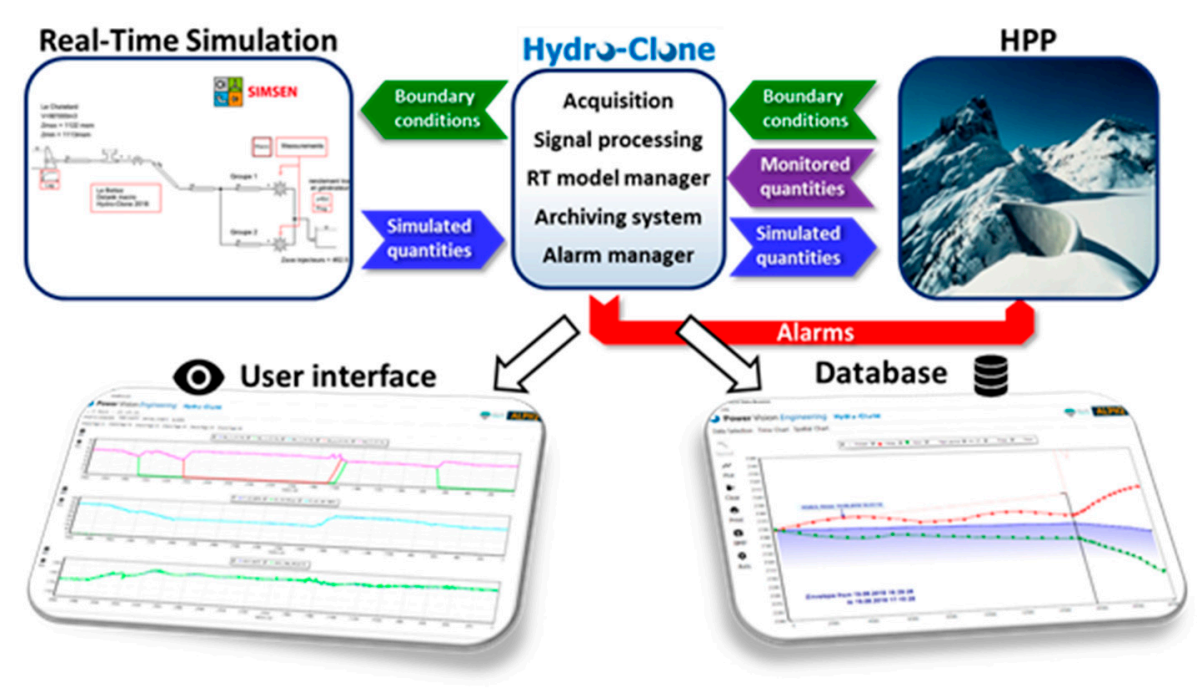

(a)

Figure 7. Cont. 


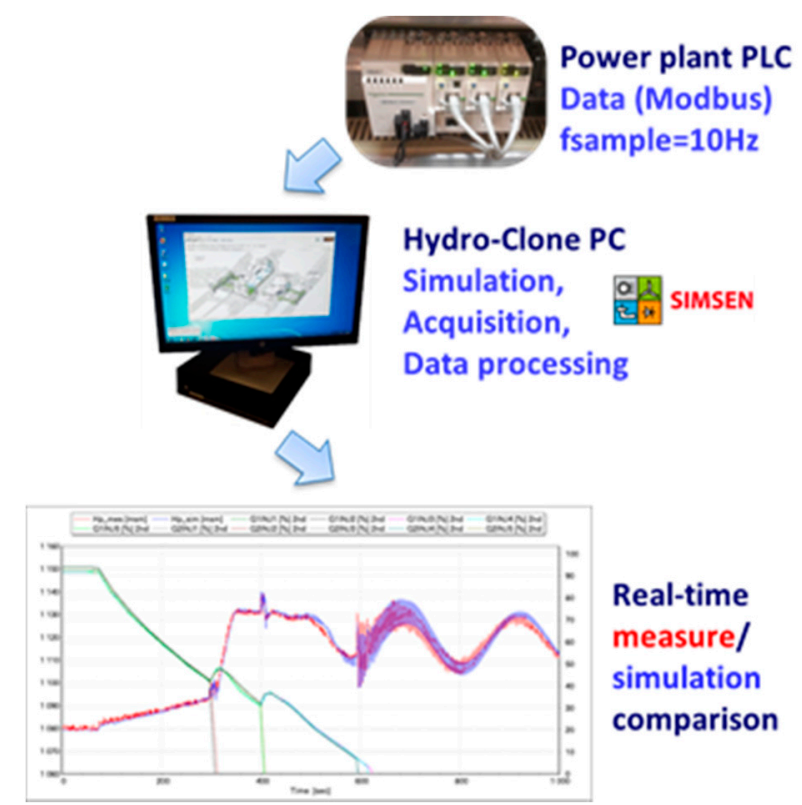

(b)

Figure 7. The general concept of Hydro-Clone with the illustration of data exchange and visualization during the real-time simulation (a) and its practical implementation with the hydropower plant (HPP) controller (b).

\subsection{La Bâtiaz Hydropower Scheme}

The objects of this case study were Châtelard-Vallorcine and La Bâtiaz power plants, which operate in cascade. The entire complex collects water from the Mont Blanc massif, which is channeled to the Emosson reservoir. The maximum gross head is $659.5 \mathrm{~m}$. La Bâtiaz power plant produces energy using two vertical Pelton units of $100 \mathrm{MW}$ each (200 MW in total), powered by an abduction system consisting of an upper reservoir, a headrace tunnel of $9973 \mathrm{~m}$ long and $3.5 \mathrm{~m}$ in diameter, a surge tank with lower and upper expansion chambers, an inclined penstock of $1253 \mathrm{~m}$ long with a diameter of 2.7 to $2.4 \mathrm{~m}$, and a manifold, see Figure 8. The SIMSEN model of this HPP used in Hydro-Clone has been thoroughly validated with on-site tests that included an emergency shutdown of a unit operating at maximum power [25]. It is worth mentioning that Electricite d'Emosson SA contributes to both primary and secondary grid frequency control services also known, respectively, as frequency containment reserve (FCR) and frequency restoration reserve (FRR) [28].

\subsection{Real-Time Computation and Validation of Penstock Stress}

By reproducing the hydraulic transients of the power plant, Hydro-Clone provides information on pressure variations all along the waterways, which is a powerful tool for evaluating the fatigue of penstocks, provided that the stresses can be correlated with these pressure variations, either based on analytical models or with more advanced modeling. In principle, the relationship between the pipe internal pressure $p$ and stress $\sigma$ at any point, $\Delta \sigma=f(\Delta p)$, could be determined using finite element modeling (FEM) for each detail, taking into account their geometry and embedding conditions. This approach has been applied to the penstock protection valve, situated just downstream of the surge tank, where the stress was both measured with a strain gauge as well as predicted via FEM, as illustrated in Figure 9. At the location of the strain gauge, the calculation showed a good agreement with the measured stress, thus validating the relation pressure-stress derived from the FEM. The model can then be used as a basis for approximating the relationship between internal pressure and stress at other locations, particularly in critical regions where stress concentrations occur. In addition, FEM allows the surface stress to be determined near the welded details and extrapolated to the hot spots of the component to be evaluated. The same approach was applied to the Y-junction of the collector at 
the bottom of the penstock, where the simulation again gave stress values in good agreement with the measurement. For the portions of the pressure shaft with steel lining embedded in concrete and surrounded by the rock mass, advanced models of pressure shafts developed by Hachem and Schleiss can be used to deduce the stress within steel lining considering the surrounding effects, see [29].
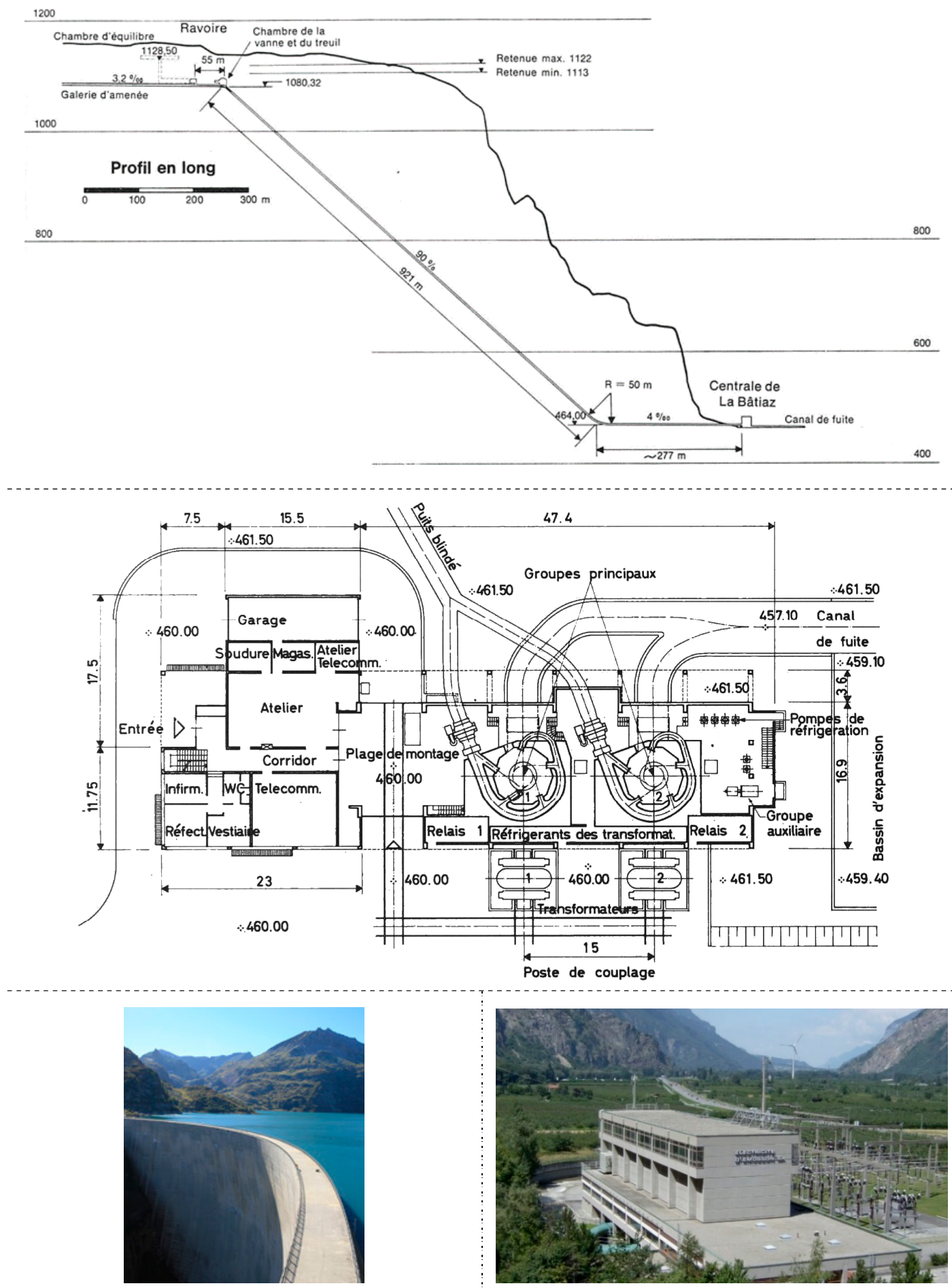

Figure 8. Top: layout of the penstock (1.2 km long) and top view of the $2 \times 100 \mathrm{MW}$ Pelton units with the manifold. Bottom: photos of the Emosson dam and La Bâtiaz power plant. 


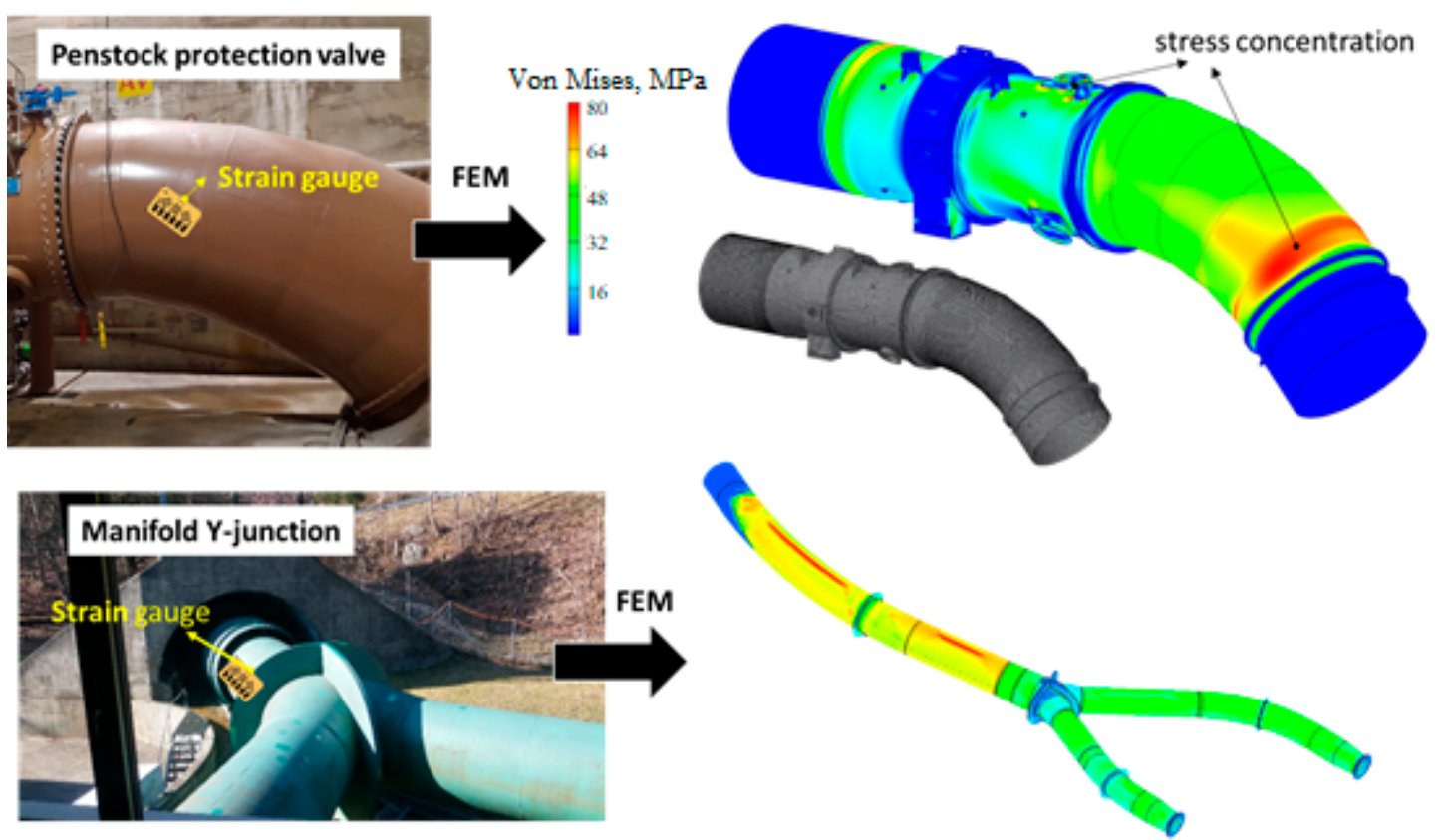

Figure 9. Top: picture of the penstock protection valve with a schematic view of the strain gauge location (left) and FEM model of the valve with the von Mises stresses (right). Bottom: picture of the manifold Y-junction with the location of the strain gauge as well as the corresponding FEM model of the element.

While FEM allows the relationship between internal pressure and stress to be obtained anywhere, it is time-consuming and should be reserved for complex geometric structures, such as valves and bifurcations. In the case of a straight open-air penstock, however, it is fair to assume that the hoop stress equation for thin shells is adequate. In order to test the validity of this hypothesis, the stress at the bottom of the penstock was measured using HBM Y 81 6/350 rosette strain gages, mounted on a straight segment of the pipe, in front of the Y-junction of the manifold. Figure 10 shows a comparison between the measured stress, obtained with an acquisition sampling rate of $10 \mathrm{~Hz}$, at the bottom of the penstock and the value simulated by Hydro-Clone using the hoop stress equation. The agreement between the measured and simulated values is remarkable, with the maximum difference between the two signals never exceeding $1 \%$, and most of the time even less than $0.2 \%$. This validates the approach described above and demonstrates that stress variations can be accurately deduced from the transient pressures simulated by Hydro-Clone.
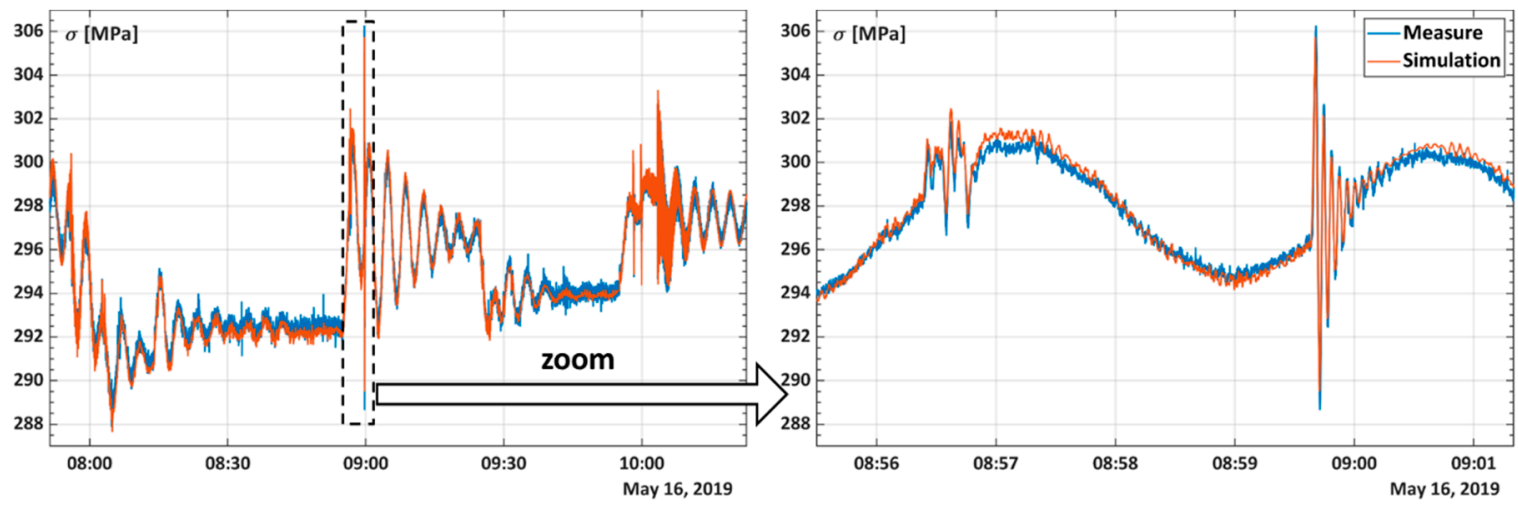

Figure 10. Comparison of the measured stress at the penstock bottom (blue line) with the simulated value calculated by Hydro-Clone (orange line). 


\subsection{Fatigue Assessment}

The overall methodology of the penstock fatigue monitoring concept is illustrated in Figure 11. The Hydro-Clone system provides real-time knowledge of transient pressures along several nodes along the penstock. These transient pressures are then converted into stress variation, using either the hoop stress equation for open-air penstocks or a more complex relation which can be derived from FEM for specific details where the hoop stress equation for thin shells is not valid [30]. Following the ASTM standard [31], the number of fatigue cycles contained in the load-time history is determined by applying a rain flow cycle counting algorithm [32]. The cumulative damage for each penstock segment is then tallied using Miner's rule in combination with the appropriate S-N curve (also known as Wöhler's curve), considering a fatigue limit of $11 \mathrm{MPa}$ in accordance with BS7910 standard [33], yielding the damage index profile along the penstock.

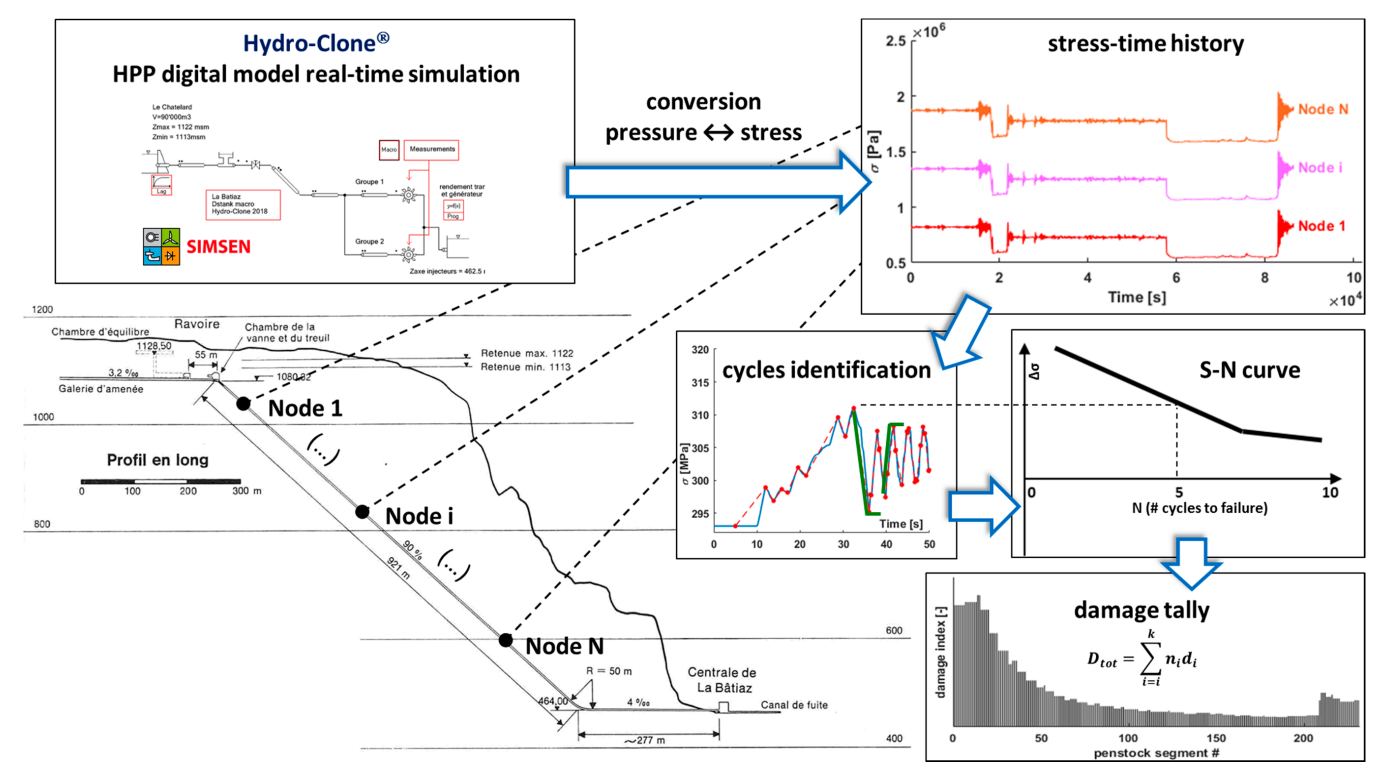

Figure 11. The overall methodology of the penstock fatigue monitoring concept.

Figure 12 shows the accumulated damage index along the penstock for the cases A, B, C and D described in Table 1 with the consideration of a fatigue limit in the damage tally, the rock contribution being neglected. In this figure, the top of the penstock corresponds to the origin of the $x$-axis, the turbines being located on the $1253 \mathrm{~m}$ abscissa. All results are presented in terms of relative damage index compared to the reference case A, i.e., the maximum damage index along the penstock obtained for case A is used to normalize the results. This allows for easy comparison of the fatigue load of the different operating modes.

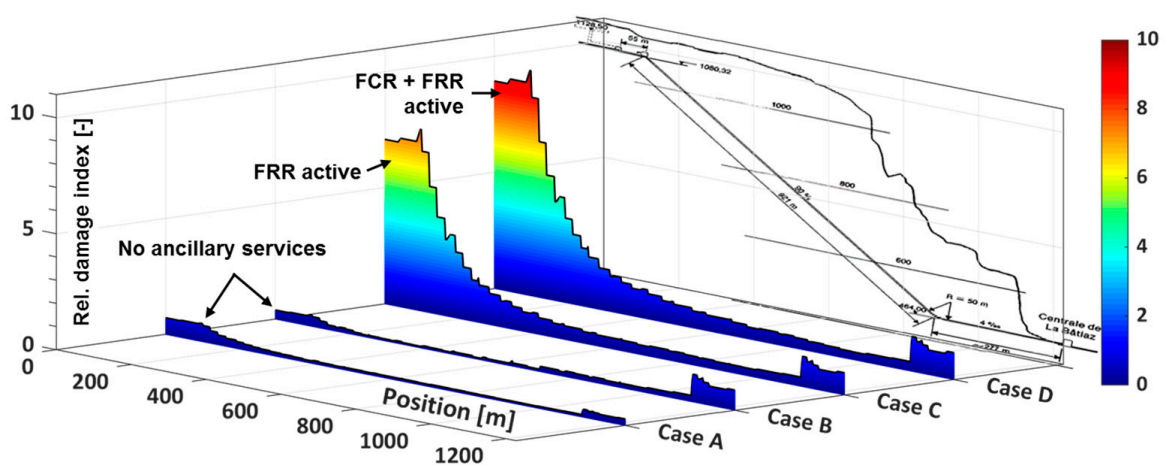

Figure 12. Comparison of the relative accumulated damage index (z-axis) along the penstock (01200 $\mathrm{m})$ for $24 \mathrm{~h}$ of exploitation with the different operation modes described in Table 1. 
Table 1. Description of the four reference cases representative of the different modes of operation.

\begin{tabular}{cc}
\hline Case & Description \\
\hline A & 24 h without ancillary service, no simultaneous stopping of both units \\
B & 24 h without ancillary service, with two starts and stops of unit 2 \\
C & 24 h with only secondary control service (FRR) \\
D & 24h with primary and secondary control services (FCR + FRR) \\
\hline
\end{tabular}

The results in Figure 12 highlight the considerable impact of ancillary services provision on penstock fatigue solicitation. The supply of primary (FCR) and secondary (FRR) control leads to a damage index about 10 times more important than without ancillary service. In terms of service life, this results in penstock fatigue wear $10 \times$ faster when these services are active. The maximal damage index is obtained for the case with both primary and secondary control services. The profile of the damage index shows that the upper part of the penstock, where the stress to pressure ratio is most important, is the most prone to fatigue [34]. The results also suggest that the major contribution of the ancillary services to the penstock fatigue comes from the secondary control (FRR). This section presented the FEM analysis of the penstock of the La Bâtiaz power plant, summarizing the information presented in [34] that represents the context of this case study. Refer to [34] for further details (all the figures have been realized by the authors of this section).

Such a digitalization system can be further used to optimize the parameters of the turbine governor in order to reduce the penstock steel lining fatigue and to find the best trade-off that fulfills the FCR and FRR ancillary services specification while improving the penstock lifetime. Another key advantage of this system is the identification of the penstock portion that is the most prone to fatigue. This information can be used to guide the experts during penstock inspections and thus focus on the most critical zones of interest.

\section{Better Management by Digitalization Software and De-Sedimentation Techniques}

The rational use of the water resource, the increase of efficiency, flexibility of generation, integration with other energy sources [35] and a reduced impact on ecosystems represent the main pillars to design hydropower of the future. This paper describes three software tools for the better management of hydropower plants, plus an example of best practice in the sediment management sector, performed by Enel Green Power, an Italian multi-utility.

\subsection{HydEA}

The growing global energy demand and the simultaneous need to increase the penetration of renewable energy sources pushes manufacturers to study methods to carry out the exploitation of existing plants in a more effective way. The algorithms developed within the HydEA (Hydro Efficiency Analysis) project were inserted in this context, to increase the overall efficiency of hydroelectric plants, hence extracting more energy from water (Figure 13). The expected increase in efficiency, through a more rational use of the resources and therefore without the need for large investments, was obtained by data-driven approaches to the operating data of the plants: it is possible to maximize the value thanks to the latest advanced analytics and data management software tools [7]. The data were managed and exploited as a real asset thanks to the integration between a mathematical approach and engineering knowledge. HydEA is a platform where an advanced algorithm analyzes the behavior of the plant and elaborates a Reference Model of the performance characteristics of the generation units: this allows for the real-time detection of deviations from the expected values, allowing us to immediately deal with anomalies that cause inefficiency and therefore not optimal water use. It is also possible to recognize, through the recalculation of the models at fixed intervals, very slow decay of the system performance. The same models also allow HydEA to develop algorithms capable of maximizing the overall plant efficiency, through the best combination of operating turbines and taking into account the pressure drops in the hydraulic networks, while still maintaining the production 
schedule or the hydraulic flow to be processed. The first real application of this platform, on an Italian hydroelectric plant, increased the production of the plant by $1.2 \%$ on an annual basis, by subdividing the load on the generation units.

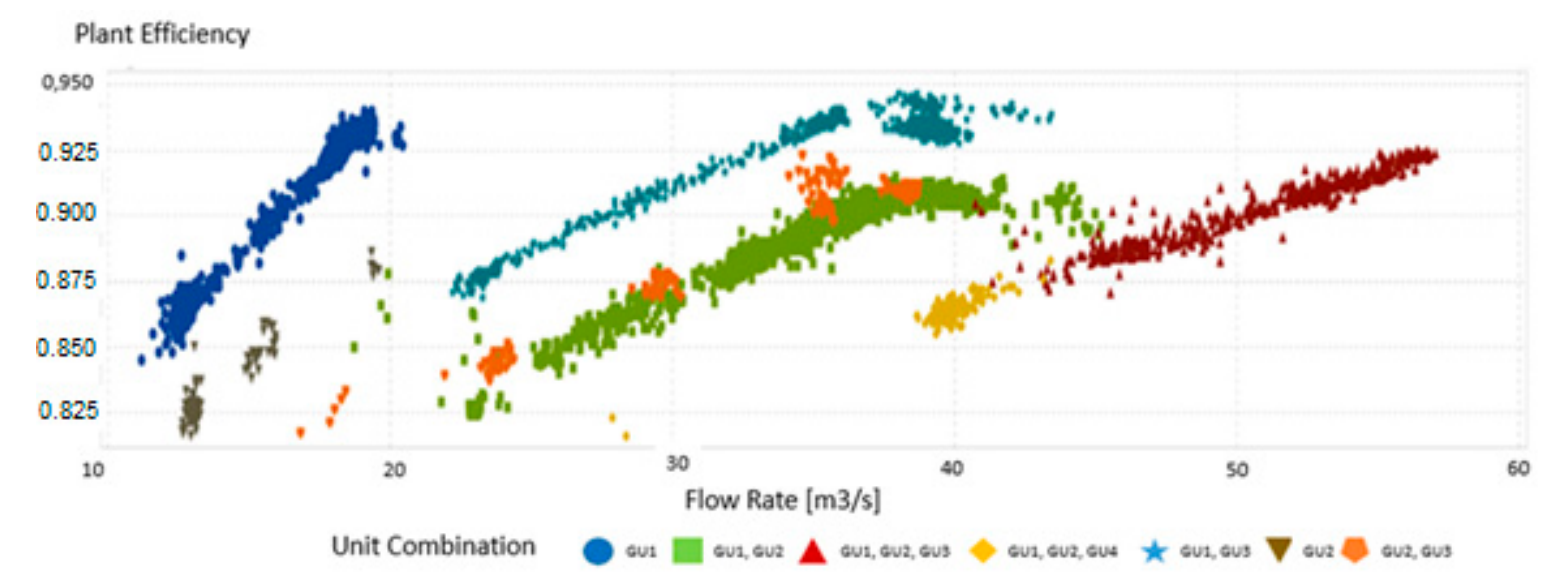

Figure 13. HydEA (Hydro Efficiency Analysis) output example applied to a power plant with four Generation Units (GU).

\subsection{Efficiency and Combination Curve Optimization (ECCO)—Sauzalito Plant in Chile}

ECCO (Efficiency and Combination Curve Optimization) is an algorithm that operates on the speed regulator and was developed to optimize the performance of Kaplan hydraulic turbines. It tracks the relationship between the opening of the distributor and the corresponding position of the impeller blades (conjugation) for each operating point of the unit. The system makes small variations in the blade opening and determines the conjugation with greater efficiency for each load condition. These points are recorded in a database and the optimal operating curve is obtained.

Sauzalito's ECCO was developed by REIVAX, the same manufacturer of the plant voltage and speed regulator. It consists of a control and data acquisition unit plus a process screen to obtain the results of its operation (Figure 14). Key complementary elements for this system are the measurement of the level in the load chamber and the turbine flow, which allows for an evaluation of the $P / Q$ performance, where $P$ is the power output (MW) and $Q$ is the flow rate $\left(\mathrm{m}^{3} / \mathrm{s}\right)$. The ECCO system has two modes of operation, Unit in Operation Mode and Unit in Testing Mode. When the unit is in Operation, a Local Search Optimizer, by means of an algorithm that considers the generated power (MW) and the generation flow $\left(\mathrm{m}^{3} / \mathrm{s}\right)$, performs an online monitoring of the opening of the distributor and of the impeller blades (interrelation called conjugation) making small variations in their opening percentage and looking for the most efficient operating point. These points are registered in a database. The test mode has a Scan Optimizer system that, using the points obtained from the operation mode (database), allows us to build the optimal combination curve for the operation, for each load condition and flow rate.

The better turbine conjugation curve translates into increased machine efficiencies between $0.5 \%$ and $0.8 \%$, depending on the operating condition of the unit. The maximum benefit is achieved at high loads. Annual earnings correspond to an additional generation for efficiency. Thus, the ECCO in Sauzalito has produced an additional $585 \mathrm{MWh}$ in the last two years.

- Annual Energy 2018 = 63 GWh; Additional Energy: 315 MWh (0.5\% of greater efficiency).

- Annual Energy 2019 = 54 GWh; Additional Energy: 270 MWh. 


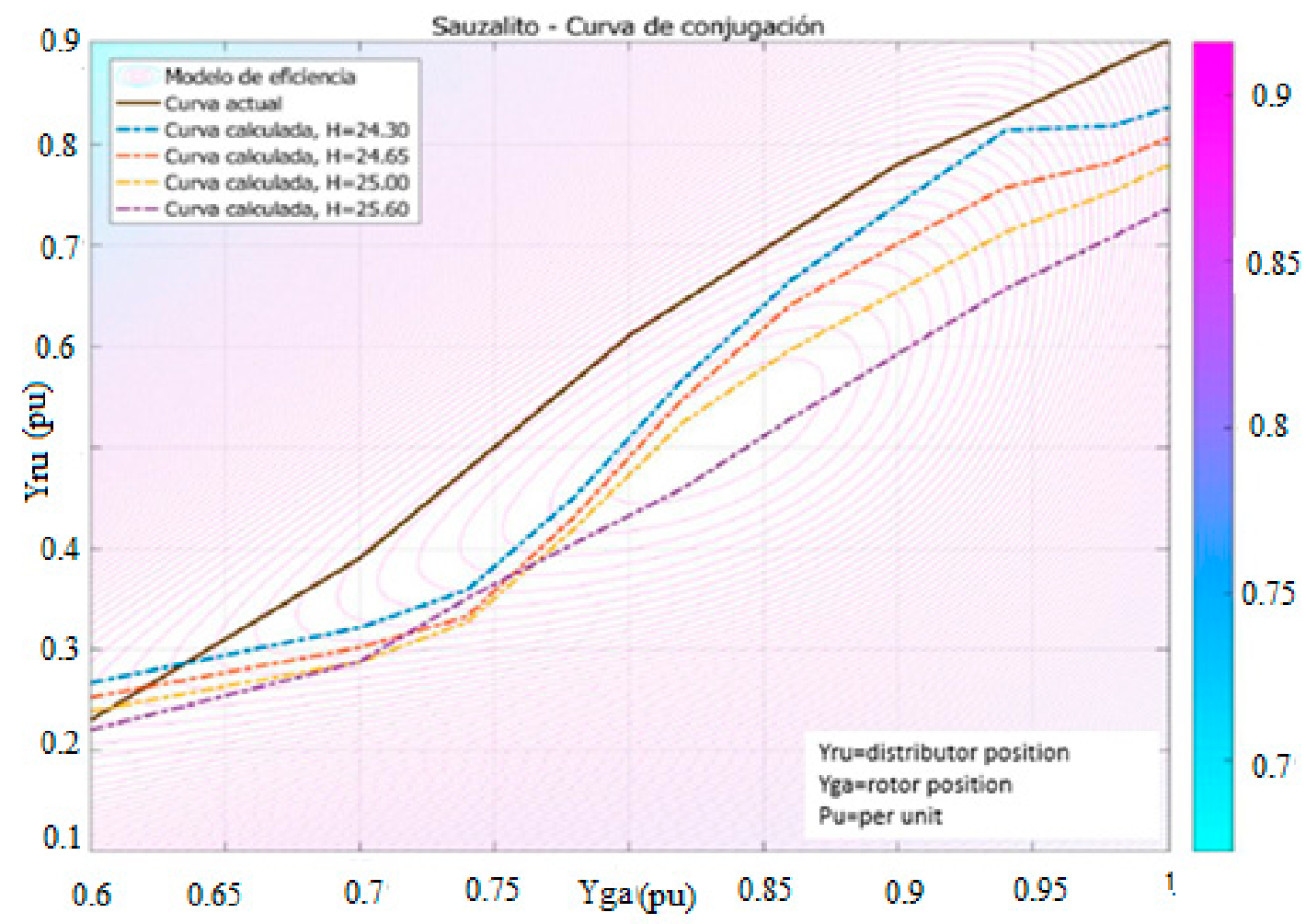

Figure 14. Sauzalito closing curves of rotor and distributor. In the legend, the efficiencies are shown.

\subsection{HYDRA-Hydrological Module for GIS Portal}

Hydro O\&M is currently developing a dedicated GIS Portal software module for providing $+72 \mathrm{~h}$ ahead previsions of water resource availability within the hydrographic basins of Enel Green Power (EGP) at the global level, for facilitating production planning processes and management of emergency flooding events. The specific module, that will operate in combination with a meteorological data service providing historical, actual and forecast meteorological data at the hourly level, will be capable of:

- Modeling hydrographic basins as per an EGP internal hydrological model-Static Calculations Module.

- Calculating at hourly level water resource availability forecasts in hydrographic basins, dams and reservoirs three days ahead-Dynamic Calculations Module.

- Providing insights on prioritization of reservoirs in operation, as per the available resource and stored energy (both in real-time and forecast modality).

- Alerting in case of forecasted Hydraulic Risks-Early Warning Module.

- Calculating the times of dams overflowing, in case of Hydraulic Risk (both in real-time and forecast modality).

A global workgroup of hydrology experts in 13 countries is currently operating in "agile mode" for publishing in the GIS (Geographic Information System) Platform all necessary local geographical parameters for the configuration of the module, such as perimeters of hydrographic basins, water level, energy curves of the reservoirs. HYDRA GIS module is intended for the use of hydrology experts, in order to perform periodically the necessary "fine-tunings" of each hydrological model (in each country), indispensable for the system accurate calculations and for performing sophisticated hydrological analysis. It is also intended for the use of all O\&M hydro colleagues that are managing hydroelectric plants operation, in order to incorporate it in their daily activities of production planning and hydraulic emergency management. Finally, one of the important advantages of the specific system development in the GIS Portal environment is the "core" functionality that GIS offers to its users. Since the information is organized in layers, this allows correlating the data of HYDRA with other geographical information 
available in the system, such as hydroelectric portfolio assets details, geo-services for terrain risk analysis and hydrographic network mapping, special inspections performed with geotechnical instruments, drones, providing further added value for O\&M also "outside" of the hydrological perimeter (Figure 15).
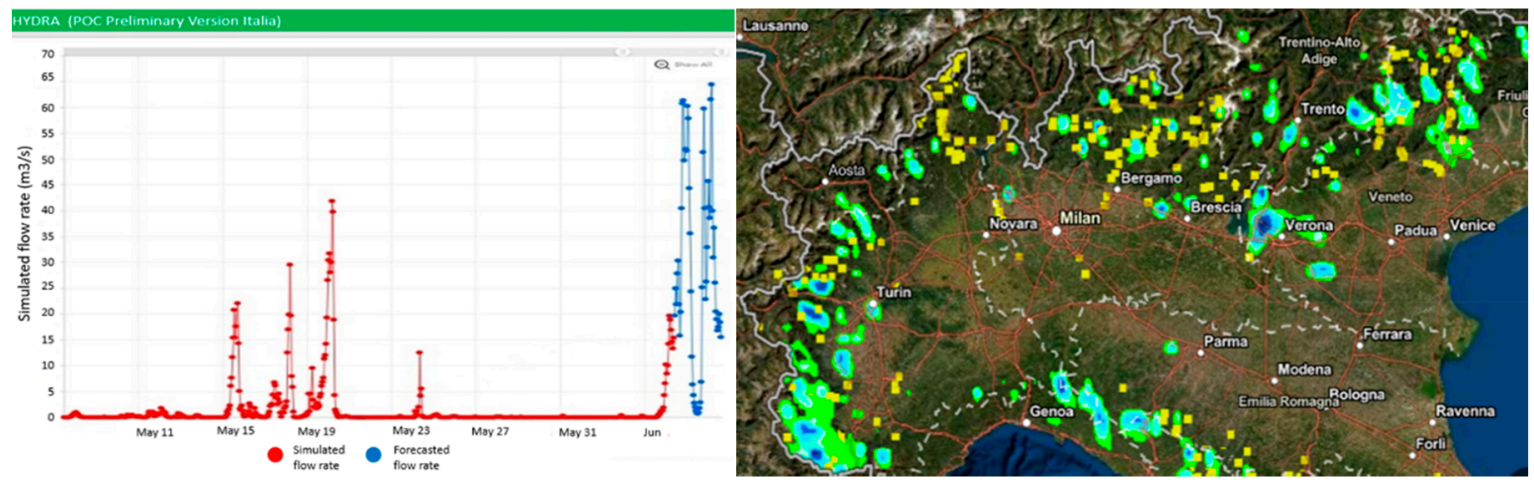

Figure 15. Examples of the HYDRA interface for inflow monitoring versus time (left) and precipitation map (right). The yellow points are the hydropower plants.

\subsection{Continuous De-Sedimentation Techniques for Hydro Power Plant Reservoirs}

Reservoir sedimentation is a process of erosion, entrainment, transportation, deposition and compaction of sediments inside reservoirs generated by dams. In unregulated, mature rivers with stable catchments, sediment processes are relatively balanced. Construction of a dam decreases flow velocities, initiating or accelerating sedimentation, resulting in the deposition of finer materials. Considering the hydropower scenario, sedimentation may lead to several issues or inefficiency, affecting the safety of dams and reducing energy production, storage, discharge capacity and flood attenuation capabilities. Enel Green Power (EGP) is responsible for the operation and maintenance of approximately 800 dams together with related upstream reservoirs, whose around $15 \%$ is affected by sedimentation phenomena with different levels of severity. For this reason, Enel Green Power has adopted the following technique for the continuous transfer of sediments. The solution replicates the river natural sediment transfer, leaving the sediment in the river system (preserving the life of the lake) trying to minimize environmental impacts, and gradual removal of all types of sediments, from cohesive clay to sand up to gravel. It also ensures low water consumption, not interfering with the hydroelectric plant management or with the ecosystem, acting without noise and in a completely automated way.

The reservoir of Pontecosi, located in the region of Tuscany (Italy) and formed after the construction of the dam on the Serchio River in 1924, was chosen to test the solution, given the estimation of an annual sediment flow rate of $20,000 \mathrm{~m}^{3}$. The sedimentation has led to a reduction of flexibility in regulation capacity, a significant environmental problem during the summer season (water scarcity) and an increase in energy production loss (up to 30\%). The solution adopted in Pontecosi, based on a fully automatic floating platform for the continuous removal of sediments, is equipped with a screw cutter at the suction unit to mobilize the sediments, which is then led through the suction line to the platform. A rotary pump is installed on the platform, which transfers the sediment through a transfer line to a point downstream of the reservoir to return to the river. The control units manage the amount of sediment to be removed and transferred with the speed of the rotary pump and the positioning of the suction unit.

The test started at the beginning of 2020 and in the end, it is expected in the autumn of the same year with a stop of the activities during the summer season for environmental norms. The initial state and the distribution of the sediments within the reservoir were calculated using bathymetry. Since the system requires a minimum water depth of about $0.5 \mathrm{~m}$, it was established to maintain the level of the reservoir at least $310 \mathrm{~m}$ above sea level. With a level of $310 \mathrm{~m}$ above sea level, the areas marked in 
yellow in Figure 16 have a water depth of less than $0.6 \mathrm{~m}$, which could limit the sediment dredging in the affected areas. The brown color instead marks the areas with a water depth of less than $1 \mathrm{~m}$.
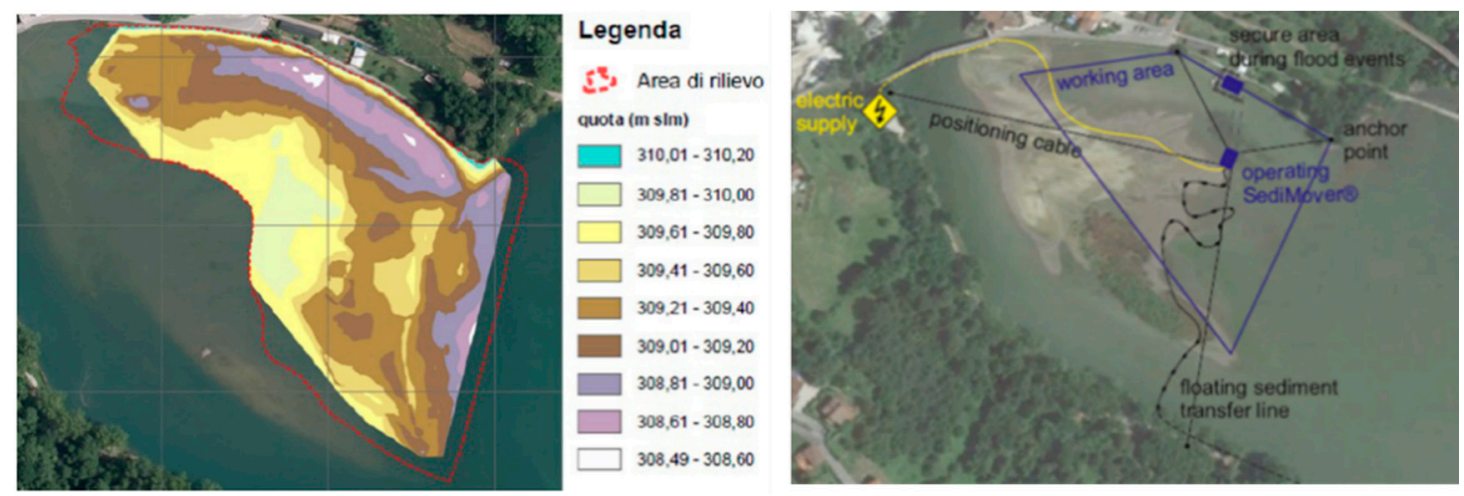

Figure 16. Results of the bathymetry to define the initial state of the sediment in Pontecosi and configuration of transfer line over the work area.

Other bathymetries are foreseen during project execution in order to monitor the rate of sediment removal compared to the initial hypothesis. To convert the quantities of transferred solids into the volume of sediment transferred downstream the Pontecosi dam, two different situations based on divergent hypothesis have been considered: (a) the upper layer of the deposits is characterized by a high water content and gas inclusions- "muddy layer"; (b) sedimentary deposits located deeper down have a more compact composition with a higher deposit density without gas inclusions- "consolidated layer". The data assumptions for the calculation of the sediment flow rate are reported in Table 2. These parameters were considered to design the entire system (i.e., sizing of the major components).

Table 2. Assumption of the characteristics of the upper part (muddy layer) and lower part sediment of Pontecosi reservoir.

\begin{tabular}{|c|c|c|c|}
\hline \multirow{2}{*}{\multicolumn{2}{|c|}{ Sediment Composition }} & Upper Part Sediment (Muddy Layer) & Lower Part Sediment \\
\hline & & $30 \%$ Solid Mass $+70 \%$ Water Mass * & $40 \%$ Solid Mass $+60 \%$ Water Mass * \\
\hline Density of solid & {$\left[\mathrm{t} / \mathrm{m}^{3}\right]$} & 2.00 & 2.38 \\
\hline Density of water & {$\left[\mathrm{t} / \mathrm{m}^{3}\right]$} & 0.98 & 1.00 \\
\hline Density of sediment & {$\left[\mathrm{t} / \mathrm{m}^{3}\right]$} & 1.16 & 1.30 \\
\hline
\end{tabular}

The value of the density of sediment (lower part) was calculated by the following formula (Equation (1)):

$$
1=0.6 \frac{\text { sediment density }}{\text { water density }}+0.4 \frac{\text { sediment density }}{\text { solid density }} \rightarrow \text { sediment density }=\frac{1}{\frac{0.6}{\text { water density }}+\frac{0.4}{\text { water density }}}
$$

The results of the first analysis illustrate how heterogeneous the sediment composition is in the considered area. Samples A02 and A03 with solid content respectively of about $27 \%$ by mass and $38 \%$ by mass broadly confirm the assumptions made in relation to the composition of sedimentary deposits. Samples A01, B01 and B02, on the other hand, have a significantly higher solid content equal to an average of $57.8 \%$ in mass. As shown in Figure 17, the difference found in sediment composition along the Pontecosi basin caused a delay in the actual sediment transfer compared to the expected value at the beginning of the project. According to the heterogeneity in the sediment composition, to improve the performance of the hydro-suction technology some technical improvements will be implemented during the summer shutdown. 


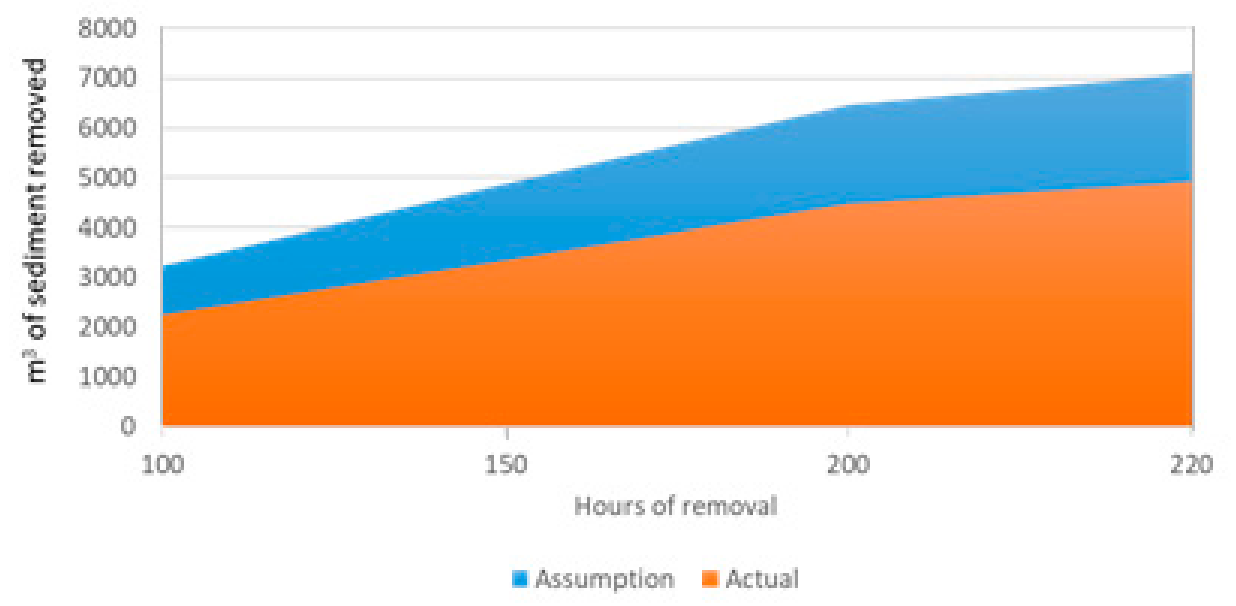

Figure 17. Comparison between the planning of sediment to be removed and the actual values removed versus the hours of operation.

\section{An Innovative Solution to Reduce the Hydropeaking Effect of Hydroelectric Power Plants. The Case of the S. Antonio Plant in Bolzano, Italy}

The S. Antonio hydroelectric plant was built in 1952 on the Talvera River in Bolzano, Italy. Its public concession expired in 2010. Eisackwerk S.r.l. has been operating the plant since winning the concession renewal tender in 2015.

The plant has a reservoir and is, therefore, able to produce electricity intermittently according to the energy needs of the national grid and according to the prices on the Italian energy exchange. It is well known that reservoir hydropower plants are the only power plants that can quickly halt production in order to store water and produce energy at a later time or deliver maximum power as needed. This flexibility makes them particularly important for the national electricity system. On the other hand, intermittent production causes a serious environmental problem, because downstream from the power plant strong and rapid river flow fluctuations ("hydropeaking") are created. These can be compared to artificial floods that drastically worsen the quality of the river environment [36]. In the specific case of S. Antonio, before Eisackwerk took over the plant, there was also a safety problem. Downstream of the plant the Talvera River flows through the most popular public park of the city of Bolzano and the artificial floods often surprised bathers, causing several drownings.

According to the regulations at the time of the concession renewal tender, Eisackwerk presented a twofold program to the Province of Bolzano to upgrade and modernize the plant and to restore and improve the environment. Solutions were therefore required that would combine increased productivity with a reduced environmental impact.

Among the main points of the expansion program, Eisackwerk planned to increase the maximum flow rate $Q$ from 15 to $18 \mathrm{~m}^{3} / \mathrm{s}$ and the maximum power generation from $72 \mathrm{MW}$ to $90 \mathrm{MW}$, in order to enhance the production flexibility of the plant. However, in accordance with its ethic of rigorous eco-sustainability, it has developed a design solution that has eliminated the hydropeaking problem. The identified solution was the construction of two large demodulation reservoirs that store the water after it has been turbined. This reservoir gradually fills up when the turbines are turning, and the plant is producing energy and it slowly empties when the plant stands still (Figure 18). The use of reservoirs is indeed one of the most effective methods to reduce hydropeaking [37]. 


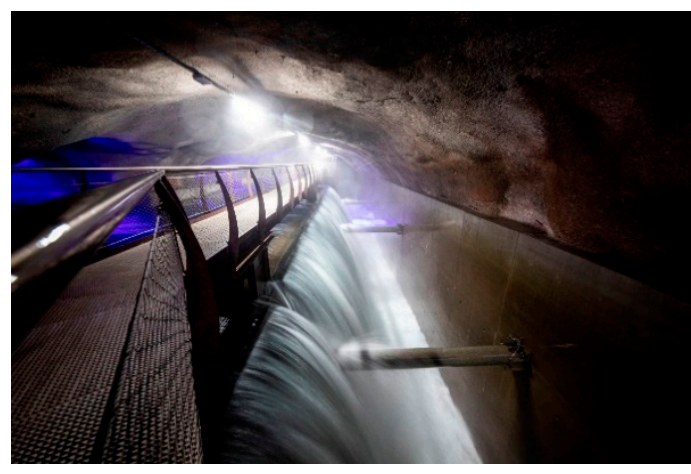

(a)

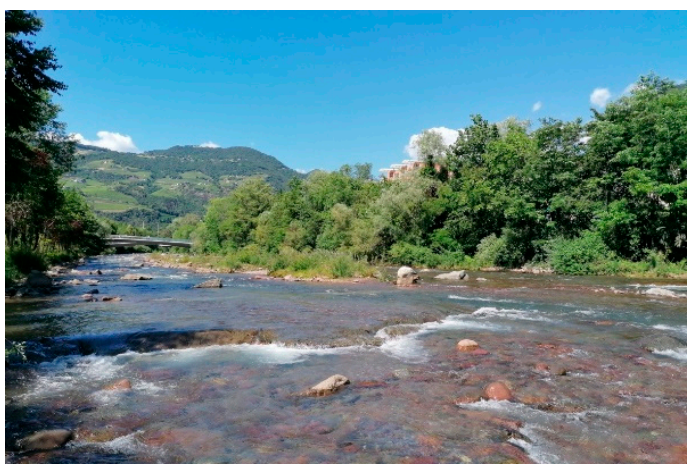

(b)

Figure 18. (a) The underground demodulation reservoir built at the S. Antonio plant in Bolzano, Italy. It was built between 2016-2018 and has a maximum capacity of approximately 95,000 $\mathrm{m}^{3}$. (b) View of the Talvera river downstream of the power plant.

Downstream of the plant the ratio between the maximum and minimum flow rate has been reduced from an unacceptable 1:15 to a value of 1:4, which has been deemed sustainable by the technicians and experts of the province of Bolzano. As a further contribution to the eco-sustainability of the plant, Eisackwerk has built the new power plant and the demodulation reservoir underground and further away from the populated area, thus eliminating the impact on the landscape and the pollution from noise and electromagnetism.

The construction of the reservoirs meant that the new Pelton turbines are located $8 \mathrm{~m}$ higher, with a loss of $1.5 \%$ of the available head difference. To recover this loss of valuable renewable energy, Eisackwerk has built an auxiliary plant-always underground-equipped with two Kaplan turbines, with a head difference equal to the height of the water in the demodulation reservoir. The reservoir is emptied by running the water through the Kaplan turbines and producing additional energy.

It should also be noted that the Eisackwerk has less water available than its predecessor. During the concession renewal process, the Province of Bolzano significantly increased the ecological outflow imposed on the plant. Nonetheless, the increase in efficiency generated by the Eisackwerk project allows the S. Antonio plant to produce approximately $15 \%$ more energy while at the same time reducing its environmental impact.

\subsection{Environmental Improvements}

The sudden changes in water flow rates related to the hydropeaking phenomenon greatly alter the ecological habitats in the river; the rapid changes in speed and water depth significantly limit the migration and settlement of fish fauna and entail a substantial risk of stranding for fish. The introduction of the demodulation reservoir reduces the impacts of hydropeaking by decreasing the maximum and increasing the minimum water release rates.

A limnological study was carried out, which analyzed the potential risk of fish stranding according to Baumann's indications [3] and the quality of the aquatic habitat according to the IFIM method [38]. The hydrodynamic characteristics of the flow rates were reproduced by the application of the two-dimensional hydrodynamic "Basement" model (research project conducted at Laboratory of Hydraulics, Hydrology and Glaciology-Zurich) with regard to the expected monthly average flow conditions along a sample section, located downstream of the S. Antonio hydropower plant. The fish stranding risk analysis was developed according to the methodology described by Baumann [36] in relation to the draining of areas with a $20 \mathrm{~cm}$ water depth. The demodulation reservoir limits the extent of the areas in the wet riverbed subject to drying up to less than $40 \%$ in all expected flow rate conditions on a monthly basis. In accordance with the limits proposed by Baumann to guarantee a low 
risk (a good risk) of stranding (Figure 19), guidelines have been defined for the emptying speed of the demodulation reservoir.

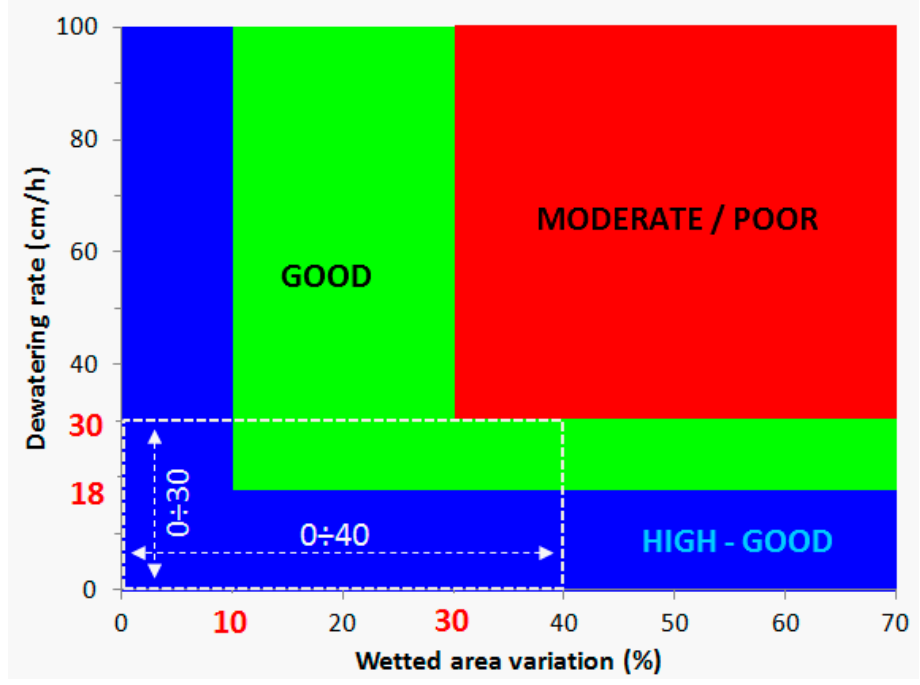

Figure 19. Overall assessment of the risk of fish stranding during the flow rate reduction phases, adapted from [39].

The minimum time for the complete reduction of the water flow rate from the maximum to the minimum flow rate refers to the time necessary to drain areas with a $20 \mathrm{~cm}$ water depth that guarantees a low risk (a good risk) of stranding within $40 \%$ of wetted area variation; the maximum dewatering rate is then limited to $30 \mathrm{~cm} / \mathrm{h}$ (Figure 19) and the minimum time of reservoirs emptying is therefore equal to $40 \mathrm{~min}$.

The ecological effects of the demodulation reservoir were assessed by verifying the habitat available for fish according to the IFIM method [38] and hereby by focusing on water speed and water depth. The suitability analysis was elaborated for the main fish species (Salmo marmoratus and Thymallus thymallus) in each simulated stretch calculation cell and the different flow rate conditions (Figure 20a,b show an example of suitable distribution conditions for adult and young Salmo marmoratus with a water flow rate from the plant of $4.0 \mathrm{~m}^{3} / \mathrm{s}$ ).

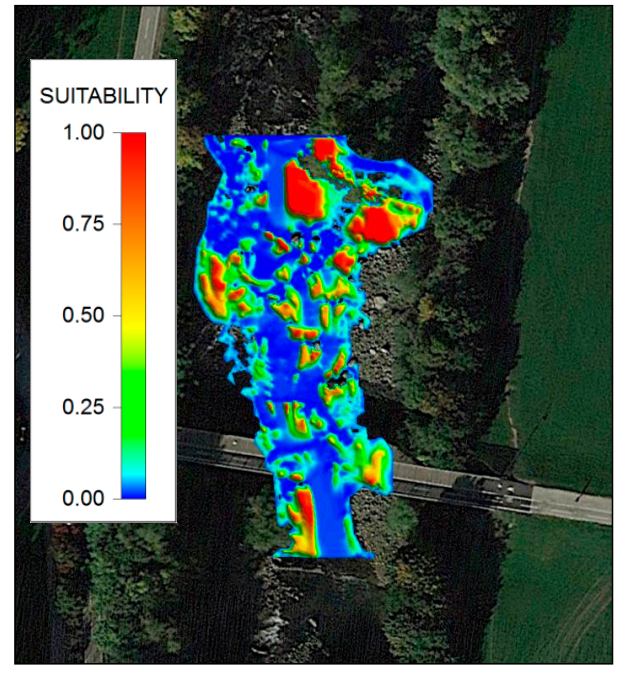

(a)

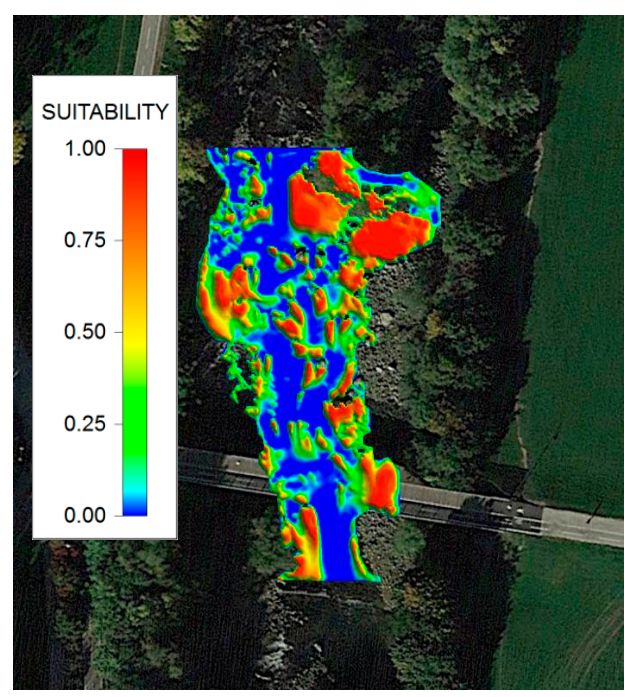

(b)

Figure 20. (a) Suitability distribution for adult Salmo marmoratus with $Q=4.0 \mathrm{~m}^{3} / \mathrm{s}$; (b) suitability distribution for young Salmo marmoratus with $Q=4.0 \mathrm{~m}^{3} / \mathrm{s}$. 
The analysis of the changes in the available habitat area WUA (weighted usable area) [40] was calculated on the entire inspected river stretch for each of the analyzed fish species (Figure 3). The results showed how the presence of the underground demodulation reservoir guarantees a general increase in habitat availability both in the minimum release conditions $\left(\mathrm{WUA}_{\min }\right)$, where the water flow rates are increased by the water released from the demodulation reservoir and in the maximum release conditions $\left(\mathrm{WUA}_{\max }\right)$, where the peak flow rates are flattened.

The analysis of the expected effects on an annual basis shows for adult Thymallus Thymallus an increase of $67.5 \%$ in $\mathrm{WUA}_{\min }$ and a reduction of $1.2 \%$ in $\mathrm{WUA}_{\max }$; for young Thymallus Thymallus an increase of $23.4 \%$ in $\mathrm{WUA}_{\min }$ and $6.3 \%$ in $\mathrm{WUA}_{\max }$ is expected. Forecast analyses performed for adult Salmo marmoratus show an increase of $14.6 \%$ in $\mathrm{WUA}_{\min }$ and $2.9 \%$ in $\mathrm{WUA}_{\max }$; for young Salmo marmoratus an increase of $3.4 \%$ in $\mathrm{WUA}_{\min }$ and $7.5 \%$ in $\mathrm{WUA}_{\max }$ is expected (Figure 21).

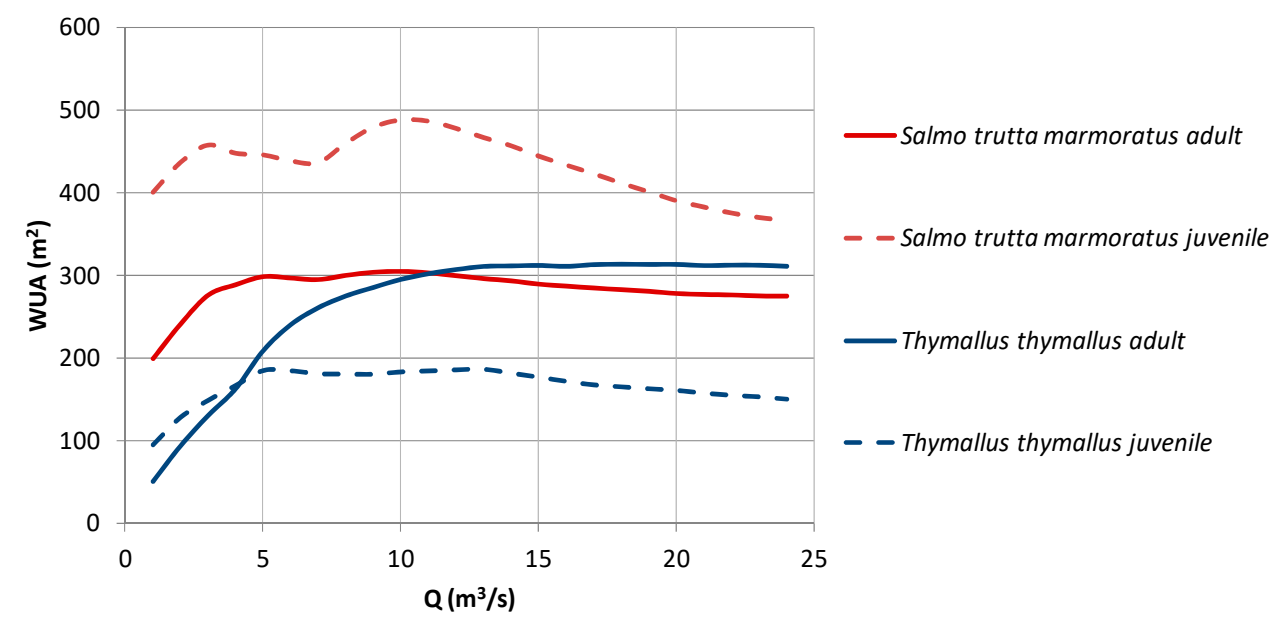

Figure 21. The resulting weighted usable area (WUA) curves for the main fish species.

\subsection{Conclusions}

Eisackwerk S.r.l. has upgraded and modernized S. Antonio hydroelectric plant using an efficient solution to reduce its environmental impact. New technical solutions have been applied to improve the productivity of the plant by increasing the maximum power generation and ensuring flexibility in electricity production. The construction of two large, underground demodulation reservoirs has led to significant environmental improvements by reducing the effects of hydropeaking downstream of the plant. To guarantee a low risk (a good risk) of standing for fish populations, guidelines have been defined for the emptying speed of the demodulation reservoir. The analysis of the changes in the available habitat area for fish showed how the presence of the underground demodulation reservoir guarantees a general increase in habitat availability.

\section{Biological Validation of Improved Direct Turbine Survival at Ice Harbor Lock and Dam}

In the Pacific Northwest, United States, many species of anadromous salmon and steelhead are listed as Threatened or Endangered under the Endangered Species Act of 1973. Anadromous fish life histories are marked by freshwater spawning and rearing, juvenile migration to the ocean, maturation to adulthood in saltwater, and adults returning to their natal freshwater streams to spawn. While dams present obstacles or impediments to fish migration, substantial changes have already been made to improve juvenile ocean-bound migration in the Columbia River Basin. In fact, according to federal studies at eight dams on the lower Columbia and Snake rivers, approximately 92 to 96 percent of juvenile salmonids pass each dam successfully, including Ice Harbor.

The U.S. Army Corps of Engineers (USACE) Ice Harbor Lock and Dam (Figure 22), located in Washington State, is one such facility focused on improvement. The dam houses six large vertical 
Kaplan turbines. Units $1-3$ are rated for $106,635 \mathrm{~kW}$ at $27.1 \mathrm{~m}$ of net head and were commissioned in 1961. Units 4-6 have a slightly higher rating of $129,752 \mathrm{~kW}$ and came online 15 years later.

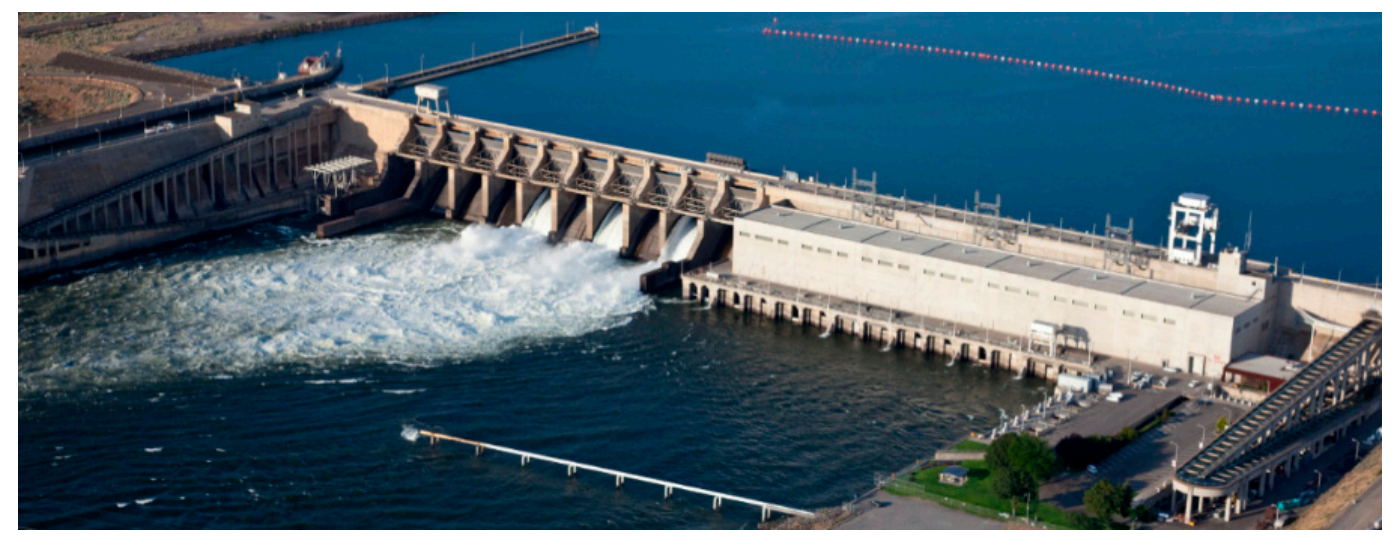

Figure 22. Ice Harbor Lock and Dam, located on the Snake River in southeast Washington State, U.S.

The USACE manages juvenile migration at Ice Harbor through several routes including mandatory spill, turbine intake bypass screens and direct passage through the operating turbines.

To improve the survival of fish passing through the turbines, the USACE initiated a collaborative design effort with Voith Hydro and the Bonneville Power Administration (BPA) in 2010 to investigate modifications to the existing Ice Harbor water passage geometries. Two replacement solutions were investigated including fixed blade and adjustable blade designs.

\subsection{Geometry Development and Evaluation}

During the geometry development, the turbine runners and associated water passageway modifications were investigated with state-of-the-art Computational Fluid Dynamics (CFD) to assess various fish passage criteria, including reducing shear, improving overall flow quality, and a target minimum nadir pressure within the water passageway of atmospheric pressure, $101 \mathrm{kPa}$. Computational results were also utilized to evaluate blade changes aimed at reducing fish contact with the runner and water passageway components. Selected geometries were manufactured for 1:25 scale physical model testing.

Physical model performance testing was conducted first at Voith Hydro's hydraulic facility in York, PA to document power, efficiency, cavitation, runaway speed, thrust and pressure pulsation data. The hydraulic shapes were then tested at the USACE Engineer Research and Development Center's Ice Harbor test facility in Vicksburg, MS to evaluate fish passage characteristics via neutrally buoyant bead tracking [41], dye visualizations and Laser Doppler Velocimetry (LDV) measurements.

\subsection{Project Schedule}

- $\quad$ Project Kick-off, Spring 2010.

- $\quad$ Start of Physical Model Testing, Spring 2011.

- Start of Fixed Blade Prototype Manufacturing.

- $\quad$ Spring 2013.

- Start of Unit 2 Prototype Installation, Winter 2017.

- Biological Testing of Unit 2, Fall 2019, Figure 23. 


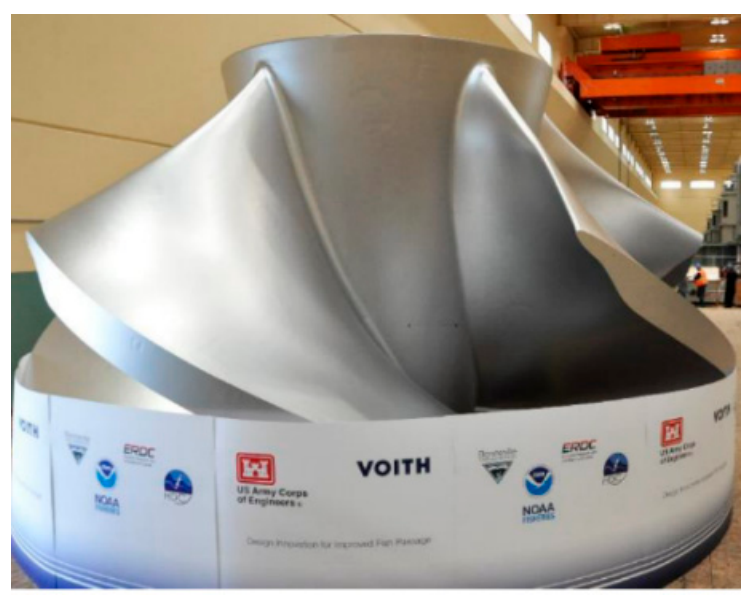

Figure 23. Fixed blade runner for Unit 2.

\subsection{Unit 2 Biological Testing}

After the final prototype geometries were manufactured and installed, a series of biological evaluations were performed on Unit 2 (Figure 23) in October 2019. Live fish were released with the following study objectives.

- Estimate direct injury and survival of juvenile Chinook Salmon passing through Ice Harbor turbine Unit 2 at three operating points, as described below in Table 3.

Table 3. Fall 2019 Ice Harbor Unit 2 operating conditions.

\begin{tabular}{ccc}
\hline Operating Points * & $\begin{array}{c}\text { Head Range } \\
{[\mathrm{m}]}\end{array}$ & $\begin{array}{c}\text { Flow Range } \\
{\left[\mathbf{m}^{3} / \mathbf{s}\right]}\end{array}$ \\
\hline $\begin{array}{c}\text { Discharge Corresponding to a 1\% Efficiency } \\
\text { Drop from the Peak Efficiency }\end{array}$ & $29.78-29.84$ & $349.7-353.1$ \\
\hline Peak Efficiency & $29.74-29.91$ & $365.3-367.3$ \\
\hline Generator Limit & $29.73-29.77$ & $384.3-385.4$ \\
\hline
\end{tabular}

* The Unit 2 runner speed and diameter is $90 \mathrm{rpm}$ and $7.1 \mathrm{~m}$, respectively. ${ }^{* *}$ During testing, changing site conditions resulted in small variations in the head and discharge for a given operating point.

- Determine type, severity and likely cause of injuries incurred to the fish passed through Unit 2.

- Test for significant differences in direct injury and survival estimates among the three turbine operating points.

- Compare the 2019 direct injury and survival results to historical study results from the 2007 evaluation of the existing adjustable blade Ice Harbor turbine Unit 3 [42].

Additionally, Sensor Fish were released [43] with live fish to capture pressure changes and acceleration data throughout the Unit 2 water passageway to validate biological design criteria.

Setup and Methodology

The fish handling, tagging, release, recapture and holding protocols were identical to the 2007 turbine Unit 3 evaluation [42]. Similarly, Sensor Fish were tagged, released and recaptured as described in Martinez et al. [43]. Treatment fish were released into each of the three Unit 2 turbine intakes from release pipes fastened to the turbine intake fish guidance screens. The location of the release pipes was modeled and selected to represent the "typical" flow path most likely followed by a fish passing through the turbine [41,44]. To minimize the potential for tagged fish to encounter injurious sheer at the release pipe exit point, the pipes were flushed with water providing similar velocity to flow 
through the turbine intake. To evaluate the effects of handling, tagging, releasing and recapturing, as well as to provide additional data on recapture probabilities, control fish were released in the tailrace near the Unit 3 draft tube exit.

Direct injury and survival were estimated at $48 \mathrm{~h}$, both including and excluding fish that were preyed upon $(\mathrm{n}=13)$ for two reasons:

- Control fish were not exposed to the same tailrace predation risk as treatment fish due to water surface releases (none of the control fish were preyed upon).

- The primary goal of the study was to assess survival and injury directly associated with the turbine passage environment.

The immediate post passage status of recaptured fish or retrieval of dislodged tags was designated as described by Skalski and Townsend [42].

\subsection{Results and Conclusions}

During testing, 1068 treatment fish were released and 1030 were recaptured. The $48 \mathrm{~h}$ survival estimates ( \pm Standard Error) excluding predation for the Lower 1\%, Peak Efficiency and Generator limit were $98.57 \pm 0.75 \%, 98.16 \pm 0.84 \%$ and $98.30 \pm 0.79 \%$, respectively. Of the recaptured treatment fish, $1.5 \%(\mathrm{n}=15)$ displayed visible injuries, the majority of which were bruising to the head and body $(0.7 \%)$ and eye damage $(0.5 \%)$. Four fish $(0.4 \%)$ experienced decapitation.

Malady-free estimates $( \pm$ Standard Error), excluding predation injuries, for Lower $1 \%$, Peak Efficiency and Generator Limit operations were $99.14 \pm 0.50 \%, 98.79 \pm 0.60 \%$ and $97.72 \pm 0.80 \%$, respectively. Survival at $48 \mathrm{~h}$ for the three operating conditions (Lower $1 \%$, Peak Efficiency and Generator Limit) ranged from 2.2 to 3.3\% higher for the new Unit 2 than at the existing adjustable blade runner tested in 2007 (Unit 3). Additionally, fewer fish were injured at Unit $2(1.5 \%)$ than at Unit $3(3.8 \%)$ and only $0.4 \%$ of Unit 2 fish were decapitated compared to $1.2 \%$ at Unit 3 . Statistical comparisons among direct injury and survival estimates for the Unit 2 operating points will be completed concurrently with results from future biological testing of the new adjustable blade design. Sensor Fish data analysis was pre-decisional as of drafting this manuscript. However, preliminary results suggest that the target minimum nadir pressure of $101 \mathrm{kPa}$ was largely realized, as well as a reduction in Sensor Fish contacts with structures within the turbine water passageway.

The 2019 Ice Harbor Unit 2 study results demonstrate exceptional survival estimates for the direct passage of migrating juveniles. While fish release methods did not directly replicate volitional fish passage, minimum nadir pressures recorded by Sensor Fish support the direct injury and survival results, suggesting the new Unit 2 design minimized the potential for barotrauma and substantially reduced other injuries. The Ice Harbor solution represents the next generation of hydro turbine technology, made possible only by extensive collaboration between design engineers and biologists during the geometry development and evaluation phase of the project.

\section{Hydropower as a Driving Force for Alpine Territorial Development}

From the simple supply of a small community, essential for its existence and survival, to the operation of the complex aqueduct, irrigation, regulation systems and use of water for the production of energy, the management of water resources represent a social opportunity, not only in response to primary and economic needs, but also in the sense of growth (or recovery) of sensitivity and cultural goals that can be important for the quality of society [45].

Hydropower that considers downstream interest and ensures environmental protection can be a very important driving force for sustainable territorial and social development. Good management of water resource and water services, profitable in the general interest and free from ideological prejudices, should preferably develop with strong territorial proximity, and in this way can constitute a durable sociological and, consequently, socio-economic opportunity, with positive effects especially on a local scale. Effects that Alpine communities need and that can be obtained stimulating and spreading two 
important factors for good policies are: competence, as knowledge of expert systems acting embedded in social life, and trust, as confidence in these expert systems [46].

The case of Maira SpA, a public-private company which since 2005 has built three hydroelectric plants $(5 \mathrm{MW})$ in the upper part of the Maira Valley, in north-western Italy, in the province of Cuneo, is described below. This experience represents a virtuous example of an alpine territory-with connotations of socio-economic marginality-which leads to a stable and positive territorial development project through the enhancement of water resources for energy (hydroelectric) on a local basis.

At the beginning of 1900, the first Italian hydroelectric plants were built in the Maira Valley, which produced, even at that time, a great boost to the socio-economic development of the valley.

The Maira Valley is now one of the most dynamic places in the western Alps, thanks to the rebirth processes after the heavy depopulation phenomenon of the 1960s-1980s. The valley, characterized by a naturalness and an environmental and landscape beauty of rare value, hosts a community of about 21,000 inhabitants on $630 \mathrm{~km}^{2}$.

\subsection{The Maira's Hydropower Plants}

Maira SpA is a public-private company established in 1998 to use water resources for local development, and since 2005 combined the construction and management of hydroelectric plants in the upper Maira valley, with the ability to generate, maintain and exploit the economic and technological results of the industrial activity entirely for the benefit of the territory in which it operates.

The company combines two main prerogatives in its institutional set-up:

- $\quad$ the ability to make available and develop technical-organizational skills.

- the interest in supporting the local socio-economy ("territorial proximity");

- consequently, the company's strategy is based on the simultaneity of three specific elements:

implementation of industrial interventions primarily aimed at water and environmental resources and renewable energy sources, in the sense of their enhancement.

- sustainability and respect for the landscape-environmental quality of the territory.

- generation and effective management of externalities for the benefit of the local community.

Three are the hydroelectric plants built and operated by Maira SpA: two are run of river plants located in the upper part of the valley, one is a plant on a municipal aqueduct.

The "Frere 2" hydroelectric plant, in operation since November 2006, uses the waters of the Unerzio stream (average and maximum flow derivable respectively $0.46 \mathrm{~m}^{3} / \mathrm{s}$ and $1.1 \mathrm{~m}^{3} / \mathrm{s}$ ) to produce about $11 \mathrm{GWh}$ annually with a hydraulic jump of $370 \mathrm{~m}$ and an installed power of $3.1 \mathrm{MW}$ (one Pelton turbine horizontal axis). The plant is well integrated into the landscape and guarantees the ecological-environmental conditions downstream of the intake structure, thanks to both to the correct design of the fish-ladder, respecting good practices and hydraulic requirements (in terms of speed, water heights, jumps and turbulences) according to the swimming capacity of the local fish fauna, and also to the regulated and monitored water releases management, together with fish restocking activities all along the stream, checked annually by biological investigations (Figure 24). 


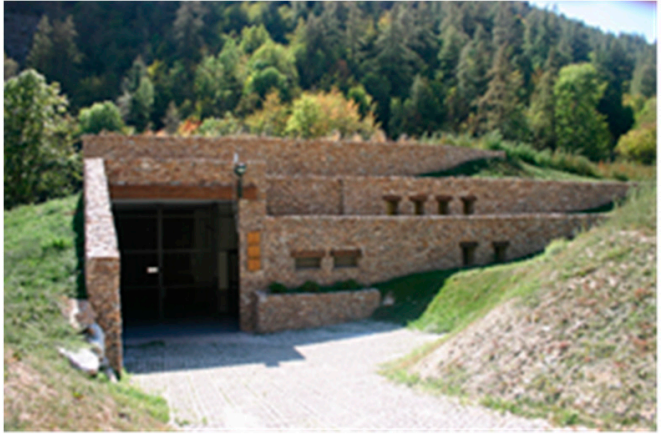

(a)

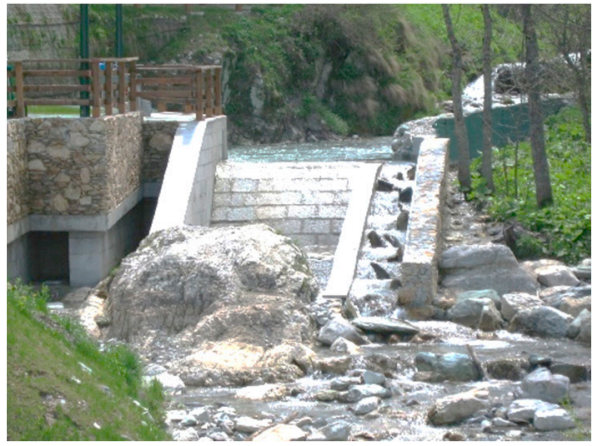

(b)

Figure 24. HPP “Frere 2"-Powerhouse (a) and intake works with the fish ladder (b).

The "Delle Fie Maurin" hydroelectric plant, in operation since December 2012, has an intake on the Rio Maurin (average and maximum flow derivable, respectively, 0.2 and $1.2 \mathrm{~m}^{3} / \mathrm{s}$ ) and produces annually about $3 \mathrm{GWh}$, thanks to a hydraulic jump of $195 \mathrm{~m}$ and an installed power of $1.9 \mathrm{MW}$ (one Pelton turbine vertical axis). The plant is almost "invisible" because both the trap intake and the powerhouse (located in a $20 \mathrm{~m}$ deep well) were built with innovative technical solutions (Figure 25).

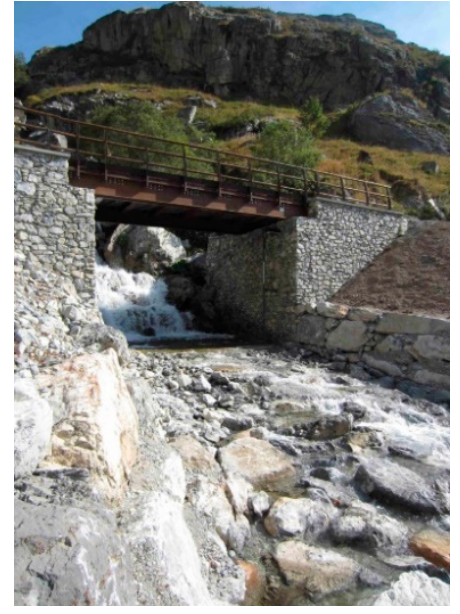

(a)

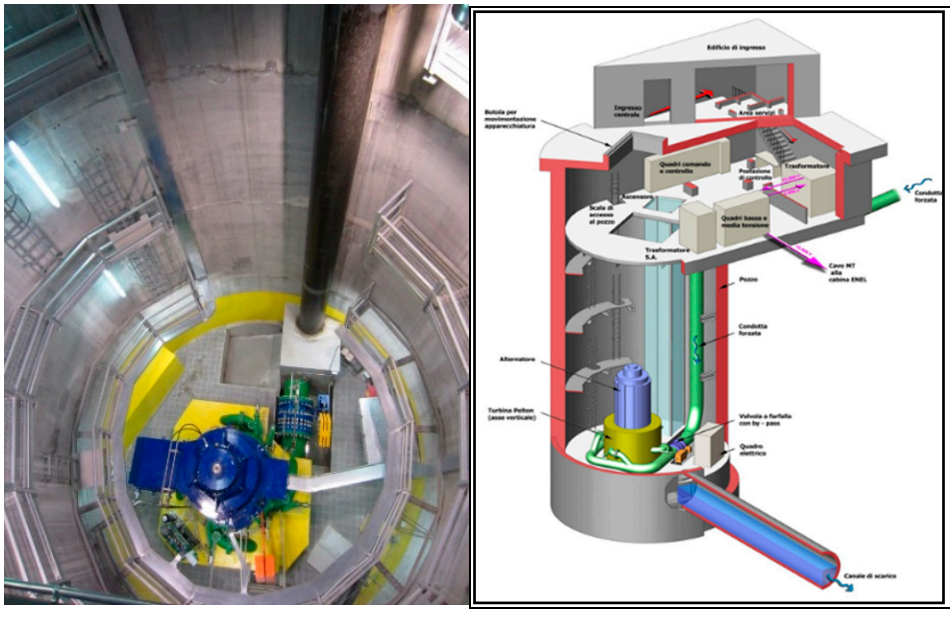

(b)

Figure 25. HPP “Delle Fie Maurin”-Trap intake under the bridge (a) and powerhouse well (b).

The "Marmora S. Anna" hydroelectric plant is located along the existing aqueduct in the Municipality of Marmora; it entered into operation at the end of 2013 and, with an average flow rate of $10 \mathrm{l} / \mathrm{s}$, a hydraulic jump of $305 \mathrm{~m}$ and $30 \mathrm{~kW}$ of nominal power, produces approximately $0.2 \mathrm{GWh}$ annually.

The three plants globally produce $15 \mathrm{GWh} /$ year, sufficient to cover the annual energy requirements of about 5000 families and to avoid more than 10,000 tons of $\mathrm{CO}_{2}$ in emission. The degree of environmental compatibility-landscaping and hydrological—of the plants is high, not only in compliance with the regional legislation on Environmental Flow, to ensure ecological functionality along the river stretch affected by the plant, but also for the added architectural value of the works on the natural landscape.

\subsection{The Territorial Development Strategy}

The economic income from energy sales is adequate to compensate the basic costs of the plants and the company, including the financial charges for the repayment of the debt and interest, and to 
cover externality plans, strategic structures for the development of the local socio-economy, the setting of new projects, as well as stakeholders' remuneration.

Territorial development enabled by the hydropower development has, for example, created non-precarious work and stimulated social positivity through various initiatives, the most relevant are:

- 2009-M.Air.A., an initiative for a new broadband internet in the upper Maira valley, with free WiFi in the main hamlets.

- 2010 - the management of the Campobase refuge (Figure 26), located at $1650 \mathrm{~m}$ a.s.l. in a magnificent alpine environment, to offer visitors overnight accommodation, food and assistance and mountain leisure activities and "school of mountain" opportunities.

- 2016-Formaira Srl for the use of local timber in a short and integrated supply chain: forestry activities, production and sale of semi-finished products for work, firewood, packaging, etc., production of wood chips, which is sold to third parties or used in thermal plants manufactured and managed by the company (Figure 27).

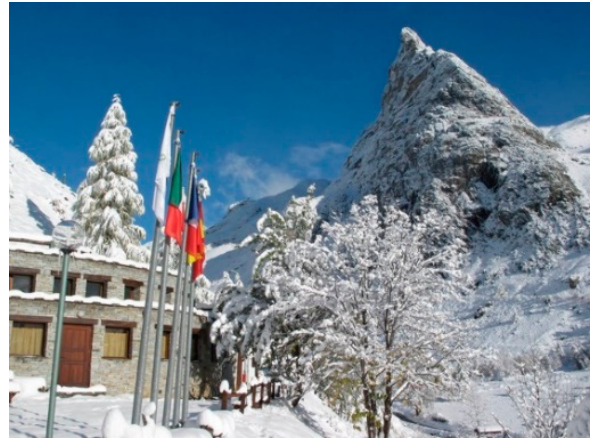

(a)

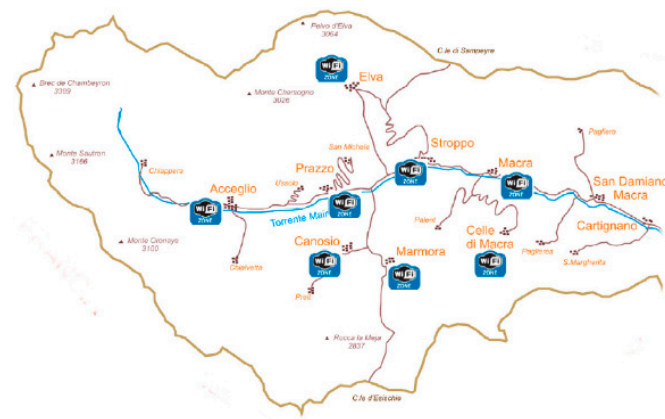

(b)

Figure 26. Campobase refuge in Chiappera (a) and broadband free hotspots map along the valley (b).

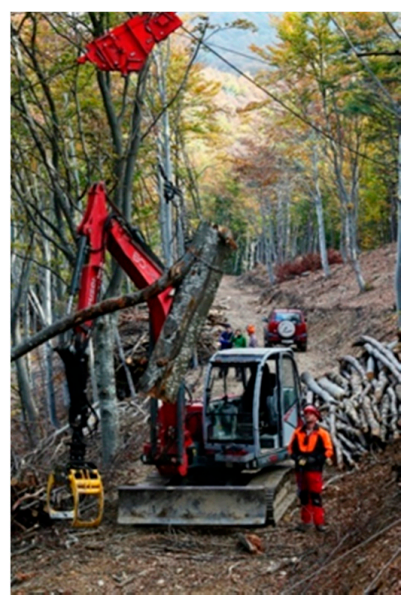

(a)
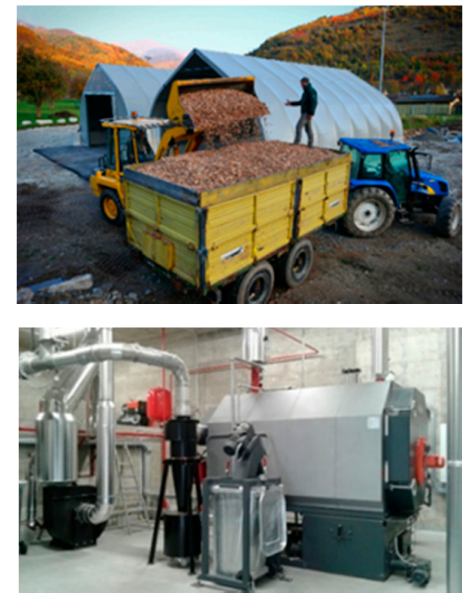

(b)

Figure 27. Energy supply chain, from wood (a) to wood-fired thermal power plant (b).

\subsection{Conclusions}

This case study shows as hydropower can sometimes address the enhancement of water and environmental resources and the provision of services and externalities of public interest, primarily in mountain areas.

The described above experience in Maira valley implements an organizational model, which-based on economic-financial sustainability, environmental sustainability and territorial 
proximity-also arouses interest outside the territory in which it originally developed. The corporate strategy is therefore open to export opportunities for the progressively acquired experience, in the ways deemed appropriate for the specific territories.

\section{Diamond Bearings for Hydrokinetic Turbines}

More than 700 million people worldwide rely exclusively on prohibitively expensive and environmentally problematic diesel generation for electricity. The cost of electricity in many remote areas is 5 to 15 times more than regional grid costs. Forward-thinking companies, like ORPC, Inc. and US Synthetic, have teamed up to utilize unique diamond bearing technology to capture and convert locally available, renewable hydrokinetic energy from the river and tidal currents into sustainable and predictable, emission-free electricity.

Successive generations of ORPC's RivGen ${ }^{\circledR}$ Power System were deployed in 2014, 2015 and 2019 in the Kvichak River at the Alaskan village of Igiugig. The remote nature of Iguigig limits the village's ability to connect to a centralized power grid or fuel supply pipeline, and diesel fuel is flown or shipped in to power the village. Using polycrystalline diamond (PCD) radial bearing technology from US Synthetic, the RivGen Power System is supplying energy daily to the village's power plant. With the installation of a second RivGen device in 2021, the project will eliminate $90 \%$ of Iguigig's diesel consumption, reduce noise pollution and decrease oil spill risk significantly.

According to researchers from the University of Alaska, "one of the most critical parts of a typical hydrokinetic device which directly affects the device's performance is the bearing-shaft assembly" [47]. Research conducted by the University of Alaska in 2014 illustrated that the bearing-shaft assembly must be able to effectively rotate in a wet, dirty and corrosive operating environment.

To overcome the challenges in Alaska's harsh marine environments, engineers at ORPC and US Synthetic developed an abrasion-resistant bearing-shaft assembly that could withstand the sediment suspended in the Kvichak River-providing a sustainable, maintenance-free solution that ensures more consistent electricity and greatly extends the operating life of the system. The bearing-shaft assembly utilizes diamond-to-diamond bearing surfaces that were designed to eliminate downtime, reduce typical wear and eliminate pre-mature failure. To date, the RivGen Power System is effectively delivering local, sustainable and clean continuous power to Igiugig Village. This case study highlights and compares bearing materials that were tested and deployed in marine environments in Alaska.

\subsection{Problem}

The dirty water passing through a hydrokinetic device poses significant challenges to any rotating equipment within the system, especially submerged bearing-shaft assemblies. Researchers at the University of Alaska looked at commonly used hydrokinetic and hydropower extraction sites in Alaska to evaluate the parameters that design engineers would need to consider when developing a wear-resistant bearing-shaft assembly. "A clear understanding of the tribological behavior of bearing-shaft assemblies in marine environments could potentially reduce the duration and frequency of interruptions resulting from worn sliding surfaces. In Alaska, marine and river sites where hydrokinetic energy extraction is being considered have high concentrations of suspended sediments due to siltation of glacial beds" [47].

Alaskan rivers, like the Kvichak River, and other coastal areas in the region contain significant amounts of damaging sediment suspended in the water including heavy silts, abrasive sands and gravel, mud and even shells and barnacles. The high concentrations of salt, acids, alkali and bacteria in the water also cause accelerated corrosion. Fluid pressures from powerful river or tidal currents are other factors that can compromise component life in submerged rotating equipment. In most cases, mechanical failure is common in Alaskan rivers and coastal regions, due to erosion and contamination in the bearing or bushing. Unfortunately, the seals that should protect a typical bearing from failure often break from abrasive contamination which rapidly brings the entire bearing-shaft assembly to a halt. 
Traditional ball bearings and bushings that fail in these conditions must be replaced or repaired, requiring the community to rely on backup energy systems and adding costly downtime to the project. Research engineers at the University of Alaska noted that operating in a harsh underwater environment, "makes accessing devices for maintenance expensive and difficult due to the unpredictable nature of weather conditions. It also exposes the sliding and contact components present in all marine energy conversion devices to unique environmental issues such as corrosion, enhanced abrasion due to contact with sediments, high loads due to water dynamics and biofouling, to name a few concerns" [47]. ORPC Chief Engineer Ryan Tyler noted, of the Igiugig project:"Because our devices are often deployed in far northern locations where winter conditions make retrieval for inspection and minor maintenance difficult if not impossible for months at a time, unplanned maintenance has big consequences. We need components to last at least a year between planned maintenance- this is the biggest reason for choosing PCD bearings" [48].

Engineers at ORPC, US Synthetic and the University of Alaska investigated numerous bearing-shaft assembly options to overcome the environmental challenges unique to this region. Some engineers investigated adding redundant ball bearing stacks and/or encasing the bearings in large sealed sleeves for protection. Other engineers tested bushings made of exotic materials, including:

1. Vesconite - an internally lubricated low friction polymer.

2. CIP Marine-a laminated composite material made of textiles impregnated with thermosetting resins and solid lubricants.

3. Feroform T814-a composite material made of woven fiber bonded with resin with polytetrafluoroethylene.

All of these conventional and unconventional methods worked to a degree, but the power of river and tidal currents, combined with the abrasive sediments within the water, still managed to break down these common counter measures. The methods of protecting traditional bearings and exotic bushings struggled to completely overcome wear problems and often introduced unnecessary complexity into the system. For example, stacking or adding multiple bearings to the bearing-shaft assembly increased the overall size of the tool—adding weight and cost to the system and reducing efficiency.

\subsection{Solution}

ORPC and US Synthetic teamed up to develop a polycrystalline diamond (PCD) bearing-shaft assembly for the RivGen Power System in the Kvichak River. The joint-development effort created a seal-less bearing-shaft assembly that included six PCD radial bearing sets and a two PCD thrust bearing set. Working together, the PCD radial and thrust bearing sets completely overcame the challenges of sediment-filled water and eliminated the need to surface the system to inspect and maintain the bearing-shaft assembly (Figure 28).

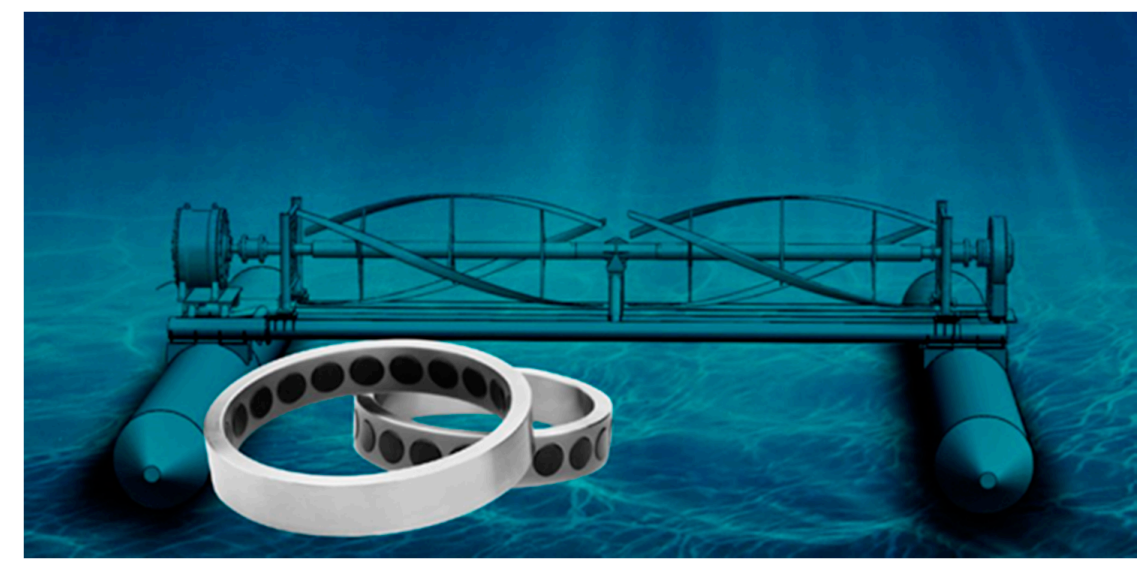

Figure 28. ORPC RivGen Power System with polycrystalline diamond radial bearings. 
Dr. Michael M. Khonsari, author and renowned tribologist from Louisiana State University, noted, "While their use is widespread in down-hole drilling applications in oil and gas, polycrystalline diamonds (PCD) have recently emerged as promising bearing materials that can withstand harsh operating conditions (high loads, high speed and high temperatures), particularly when the operating environment contains abrasive contaminants" [49].

By utilizing US Synthetic's proprietary PCD bearing technology in its RivGen Power System, ORPC leveraged:

- a process fluid-cooled bearing-shaft assembly that: (1) completely eliminated seals, (2) reduced excess component weight (3) and removed the need for contaminating lubricants and ongoing maintenance.

- A diamond material — the hardest material on earth—that easily resisted abrasive particles and damaging sediment suspended in the water.

- A sliding-element bearing surface that: (1) handled higher loads, (2) minimized operational wear (3) and delivered a lower coefficient of friction (0.01).

Relying on the inherent benefits of diamond technology, the early bearing-shaft assembly tests showed that after approximately $200 \mathrm{~h}$ in operation, the PCD bearing sets began to "wear in" and the coefficient of friction of diamond began performing like a polymer material. At these low friction levels, the PCD bearing-shaft assembly provided improved operating efficiency and eliminated excessive drag on the system's rotating elements. Cian Marnagh, ORPC's vice president of engineering noted, "We're designing for a 20-year service life" [50].

The PCD bearing-shaft assembly running in the Kvichak River simply ground up any abrasive particles that were in the water. The highly engineered bearing solution solved the problem of frequent mechanical failures caused by exposure to extreme impacts and eroding contaminants. This solution also made it possible for ORPC's RivGen Power System to shed weight by utilizing fewer and much smaller bearing packs.

\subsection{Results}

To date, the PCD bearing-shaft assembly from US Synthetic was successfully used in ORPC's RivGen devices tested in 2014 and 2015. In the newest design of the RivGen Power System launched in 2019, the PCD bearing-shaft assembly has been continuously operating without failure. When comparing other hydropower/hydrokinetic bearings and bushings, PCD bearing material delivered several clear benefits in wear, thermal conductivity, tensile strength, compressive strength, modulus of elasticity and hardness (Table 4). The properties of the PCD bearing material were compared to several other engineering materials, including tungsten carbide, silicon nitride, silicon carbide and steel (4140).

Table 4. Applied Tribology: Bearing Design and Lubrication, 3rd Edition; Source: Lingwall et al., 2013 [49].

\begin{tabular}{cccccc}
\hline \multicolumn{5}{c}{ Property Comparison: PCD with Other Engineering Materials } \\
\hline & $\begin{array}{c}\text { Thermal } \\
\text { Conductivity } \\
(\mathbf{W} / \mathbf{m} \text { K) }\end{array}$ & $\begin{array}{c}\text { Tensile } \\
\text { Strength } \\
\text { (MPa) }\end{array}$ & $\begin{array}{c}\text { Compressive } \\
\text { Strength } \\
\text { (GPa) }\end{array}$ & $\begin{array}{c}\text { Modulus of } \\
\text { Elasticity } \\
\text { (GPa) }\end{array}$ & $\begin{array}{c}\text { Hardness } \\
\text { (GPa, Koop) }\end{array}$ \\
\hline PCD & 543 & $1300-1600$ & $6.9-7.6$ & 841 & 49.8 \\
\hline Tungsten carbide & 70 & 334 & 2.68 & $669-696$ & 1.8 \\
\hline Silicon nitride & 30 & 520 & 3.45 & 296 & 1.8 \\
\hline Silicon carbide & 85 & 500 & 2.5 & 434 & 2.4 \\
\hline Steel (4140) & 42.6 & 415 & 1.6 & 205 & 0.2 \\
\hline
\end{tabular}


Researchers at the University of Alaska also compared four unique bearing/bushing materials for use in Alaskan rivers and coastal regions. These materials were tested under load for $60 \mathrm{~h}$ in freshwater and measured for wear (mm). Figure 29 outlines the benefits of PCD bearing material compared to Vesconite, CIP (ComPosIt, a laminated polymer material made by impregnating fabric with thermosetting resins) and Feroform. The test compared wear in the far, center and drive-side of the bearing/bushing.

\section{Hydrokinetic Bearing / Bushing Wear}

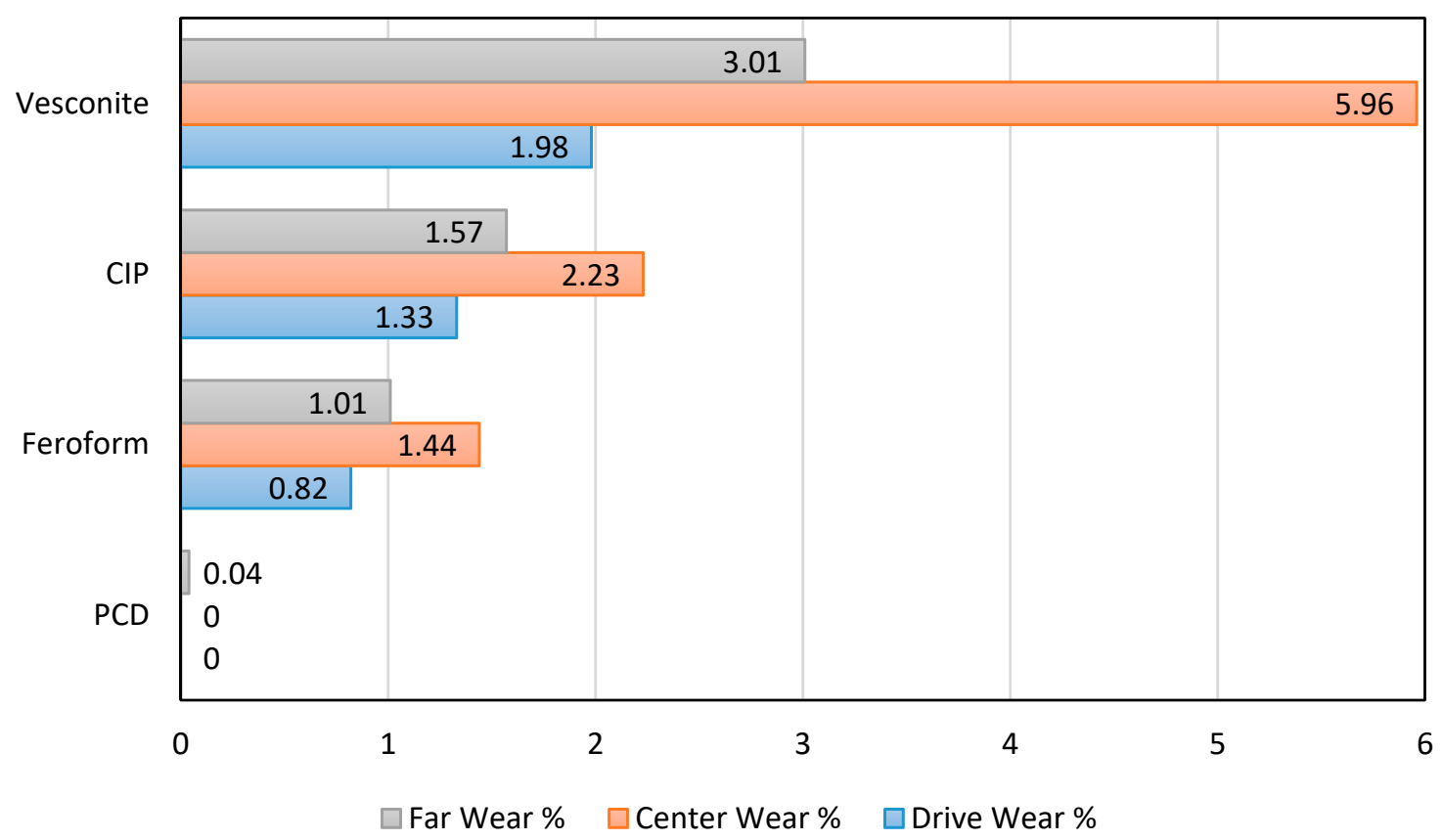

Figure 29. Experimental study of the abrasion characteristics for critical sliding components for use in hydrokinetic devices, wear test $(\mathrm{mm})$ under load over $60 \mathrm{~h}$ [47].

In conclusion, diamond bearings are a perfect fit for the ORPC RivGen Power System and are widely used in down-hole power-generation turbines. Engineers at US Synthetic also believe that axial and radial diamond bearings could significantly increase the durability and extend the life of Kaplan and Francis turbines and other submersible applications. Oil-free applications in Hydro are becoming very important and distinctive with regard to the sustainability of the plants. Among the applications relating to lubrication in the Hydro sector, one of the most critical is related to the hubs of Kaplan rotors [51]. Although the quantities of oil are not significant, the criticality is very high due to the contact with the water flow. Several experiences were made in the world, but this technology has not yet been widely implemented due to the need for complete disassembly and renewal often carried out every decade, requiring long intervention and out-of-service times. One example is the new Kaplan units of the Enel Group are all made with oil-free hubs with different technologies (metal/polymer bushings, filled with water). The most important Kaplan plant in the Enel Italia unit is Isola Serafini, 25 MW/each. Units 3 and 4 will be supplied by GE with oil-free technology and new polymer thrust bearings.

\section{The Complex Process of Hydroelectric Development of an Existing Multiple Use Reservoir}

In San Michele dei Mucchietti locality, on the Secchia River, in the Municipality of Sassuolo (MO) and Castellarano (RE), an important weir was built in the 1980s in order to provide water for irrigation to Modena and Reggio Emilia's plane through two historic channels, no longer feedable due to the lowering of the river bed. At the same time, the weir has created a reservoir of approximately 800,000 cubic meters useful to serve the two irrigation channels, one drinking water supply and 
one industrial supply. The water supplies are managed by legally different subjects with leading management in charge to Consorzio di Bonifica Emilia Centrale.

The volume is obtained in line with a river basin and laterally with a volume used exclusively in the dry season (Figure 30).

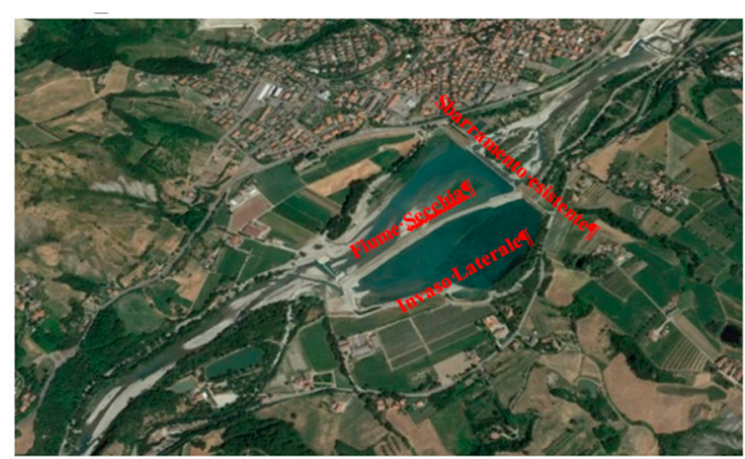

(a)

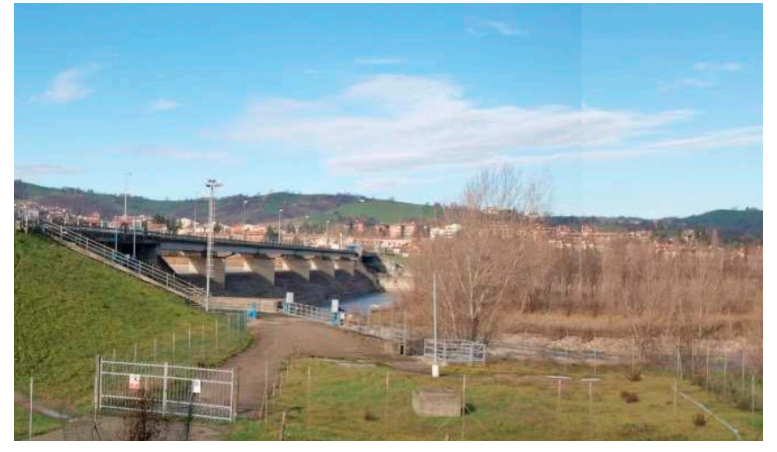

(b)

Figure 30. (a) photo of the current state-of-the-art; (b) photographic shooting of the downside of the weir.

The structure of the central part of the dam is made of a concrete body with a surface spillway and two bottom sluice gates; the shoulders both on the right and left side are earth made. The structure was not conceived to host a hydroelectric plant whose design has faced significant construction and building problems.

\subsection{The Network of Partners}

The construction of the hydroelectric plant was promoted by the company BI Energia S.r.l., Investee Company by Consorzio di Bonifica Emilia Centrale and Iren Energia S.p.A. The partners made it possible to enhance the acquired skills with specific reference to the management of hydraulic complex nodes and hydroelectric design. The management of a multiple-use structure and reservoir, despite the complexity of defining the limits and rights of each user, allowed the various users to achieve the reduction of the operating costs, also distributing them to the new co-owner of the hydroelectric plant.

\subsection{Technical Characteristics and Environmental Choices}

The hydroelectric plant is configured as a system that uses a difference in level created from the weir without water resource subtraction to the riverbed. In accordance to Article 10, paragraph 3 letter e) iii. of the Ministerial Decree of 6 July 2012, in the concession to divert water for hydroelectric use there is a severe priority defined for the different supplies with the privilege of the aqueduct use followed by irrigation, industrial and, ultimately, hydroelectric.

The main characteristics of the plant can be summarized as follows (Figure 31):

- $\quad$ average flow rate: $10.54 \mathrm{mc} / \mathrm{s}$.

- maximum flow rate: $26 \mathrm{mc} / \mathrm{s}$.

- gross head: $9.66 \mathrm{~m}$.

- nominal concession power: $998.20 \mathrm{KW}$.

- two Kaplan turbines with a vertical axis of mechanical power equal to 1207 and $603 \mathrm{KW}$ - two synchronous generators with nominal power equal to 1476 and $800 \mathrm{KVA}$. 


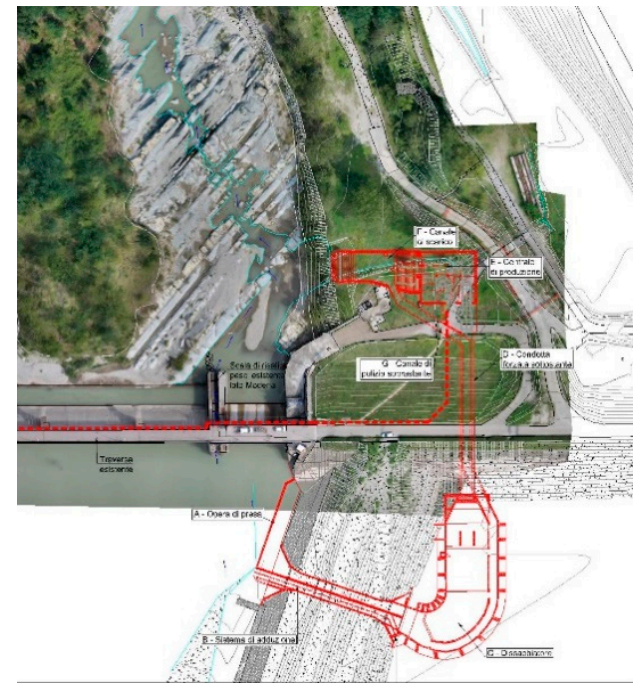

(a)

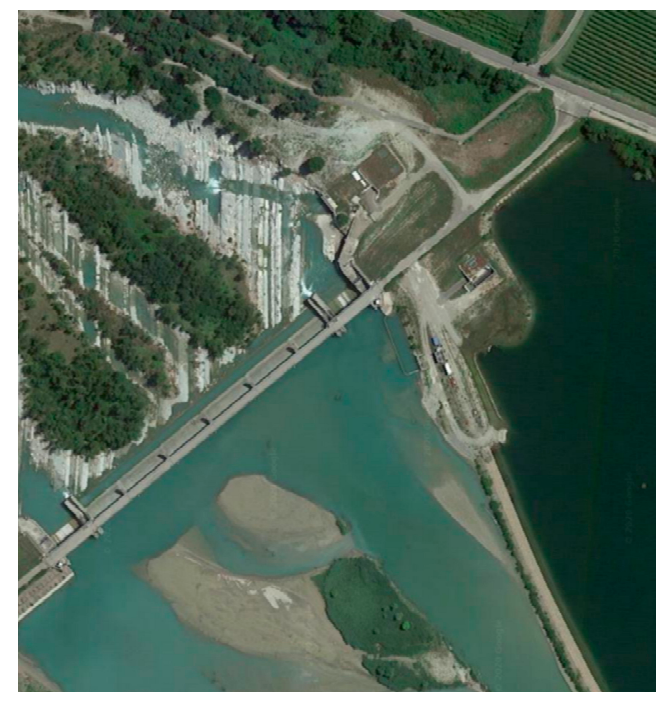

(b)

Figure 31. (a) the layout of the plant, aerial photo; (b) aerial photo of the site as built.

The plant was built by reusing part of the existing infrastructural works with reference to:

1. double connection pipes between the lateral basin and the river, which were originally oversized and used to allow the water to get back into the river from the lateral reservoir. This was done by separating one of the two pipes in order to separate the flows. The inlets have been protected on the front by a concrete sill with the function of:

stopping of the hydroelectric intake on reaching a minimum water level in the river in order to ensure the level necessary for the irrigation;

$\checkmark$ protection from the phenomenon of silting of the intake.

This design choice, compared to that initially assessed for the construction of independent intake, has allowed a substantial and quantifiable saving of 300,000 euros (300 thousands), avoiding a complex cutting operation of the bank of the lateral reservoir and its restoration of its waterproof structure.

2. Viability and access: the existing accesses, of adequate size and type, have made it possible to avoid the construction of expensive new roads and trails.

3. Embankment of the lateral reservoir: this element ensures protection from the floods of the plant, built on the back of the embankment itself. Costs were avoided for the construction of protection screens, huge stone boulders, walls higher than the two-hundred-year period flood level. A reduction in the costs of the work of 200,000 euro is estimated.

The production plant was built downstream of the shoulder of the existing weir; in order to reduce the landscape impact of the building, it was decided to build it completely underground below the countryside level, with driveway access possible only for small vehicles useful for the maintenance of the power plant equipment. Currently, the building, as better highlighted by the photographic shots of Figure 32, is integrated into the surrounding environment. 


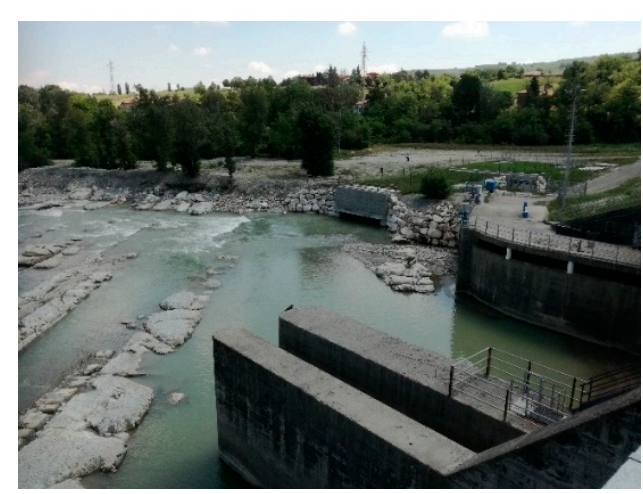

(a)

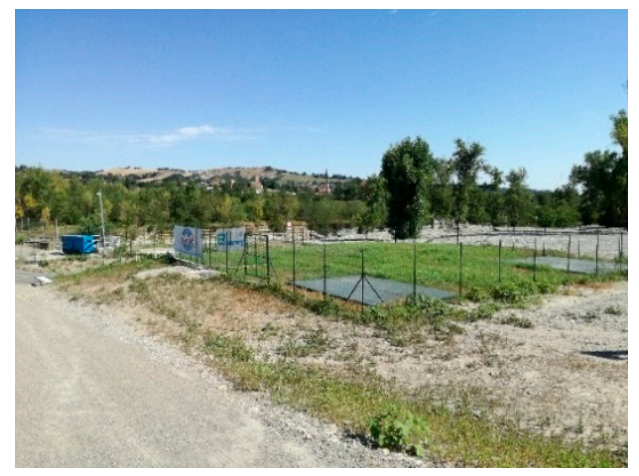

(b)

Figure 32. (a) photo from the upper side of the weir; (b) photographic shooting of the access to the building.

The environmental choice caused greater complexity and management costs since:

- It is not possible to provide for the entrance with a vehicle inside the building except with a small van; assembly and disassembly interventions need to deal with greater management complexities.

- In order to support the excavations, especially because of the proximity of the downstream face of the weir, it was necessary to adopt secant and tangent poles with higher costs than a traditional solution with a building on the ground floor estimated at $€ 90,000$. The higher cost of the work was largely compensated by a reduction in social costs and acceptance of the intervention. The existing weir is in fact an important point for cycle and pedestrian passage.

\subsection{Construction Site Design and Construction Techniques Adopted}

The design choices involved rather complex building activities, both in term of environment and location of the different items of the plant, that are briefly summarized below:

1. A need to ensure that pedestrians and cyclists could walk through the road over the weir for the entire duration of the works with safe interference management.

2. A need to ensure stability both in structural and hydraulic terms of the embankment between the lateral reservoir and the river as well as of the earth shoulder of the weir.

3. Maintenance of the existing water supplies for the entire duration of the construction site and identification of a detailed schedule for the construction of the interfering works.

4. Temporary works in order to keep the construction area site safe even with respect to flood events with return times of 200 years.

Certainly, the most peculiar aspect can be identified in structural stability and the hydraulic seal of the interfered works. Particular construction choices were made specifically with regard to the penstock which, although of limited length (about $60 \mathrm{~m}$ ), has a considerable internal diameter $(3600 \mathrm{~mm})$ and crosses the earth shoulder of the weir cutting it completely in the transverse direction. The embankment links the central body in the concrete of the weir to the adjacent slopes, and at the same time has the function of preserving the lateral water volume, guaranteeing a perfect seal both statically and hydraulically. Above the penstock, a concrete channel was installed to discharge the above desilting tank. Various technologies were evaluated in the planning stage:

1. Micro-tunneling that combines a pipe jacking technique with a remote-controlled shield machine. The thrusting force is distributed by hydraulic jacks located in the MTBM front section and operated by a thrust ring. Final injections of the interspace between pipeline and ground were planned (Figure 33a). 
2. Tunnel with traditional advancement method and consolidation treatment of the above embankment (Figure 33b).

3. Trench excavation with support of the fronts through secant poles and construction of the penstock on site (Figure 33c).

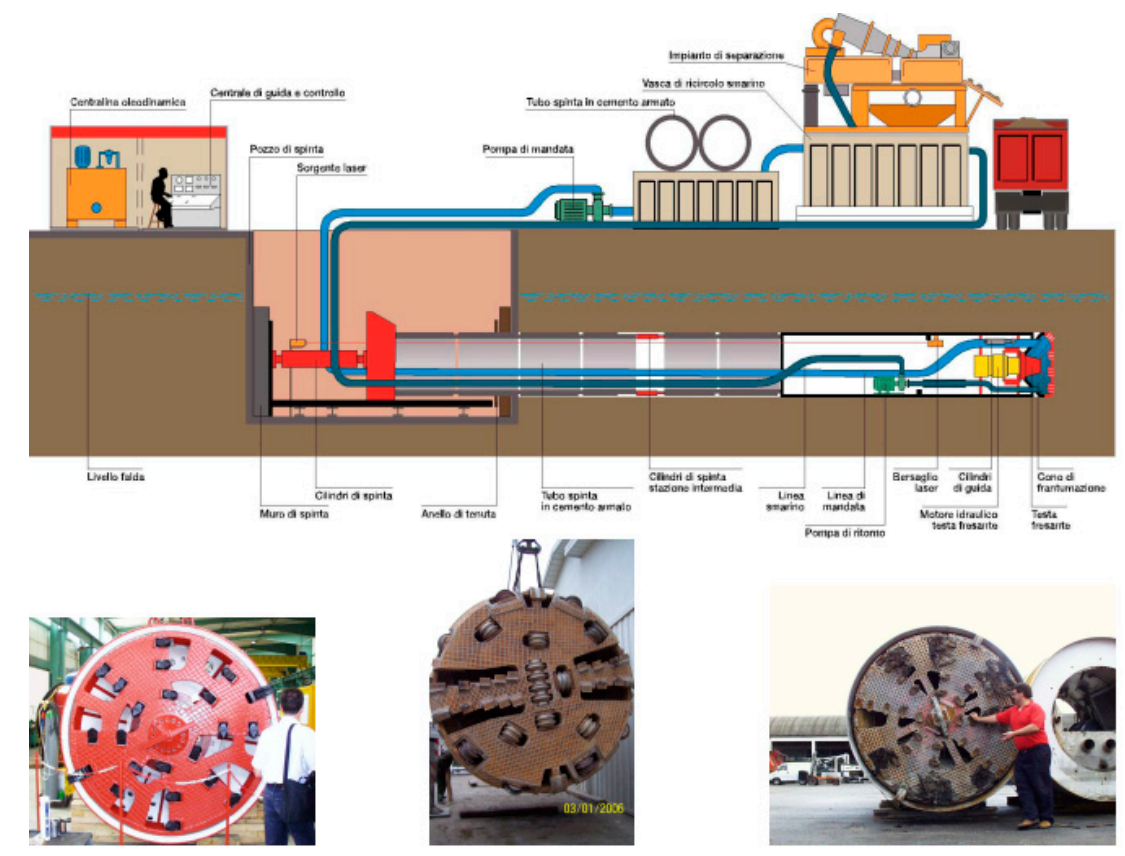

(a)

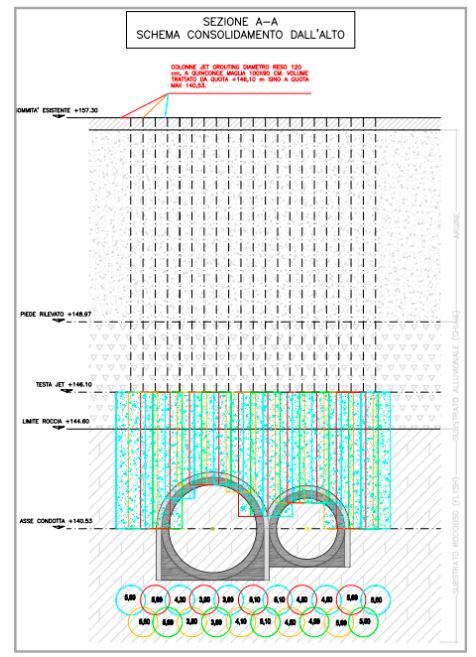

(b)

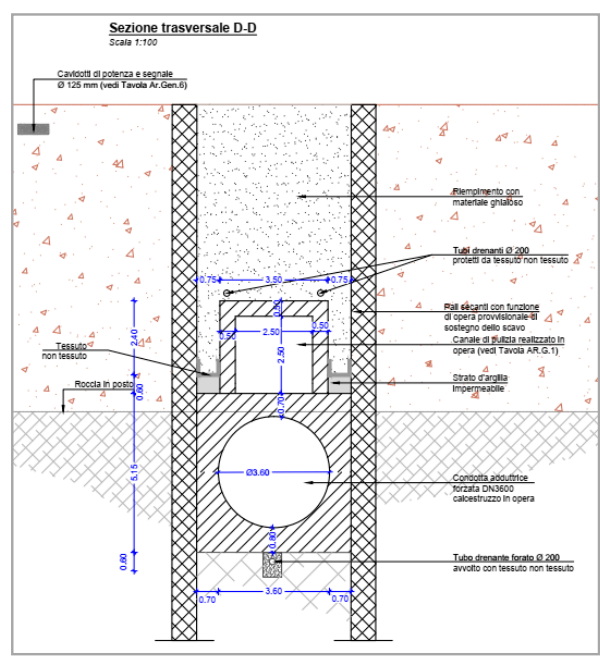

(c)

Figure 33. Different technical construction method for the penstock (a) microtunneling (ICOP/PATO);

(b) traditional tunnel (Injectsond Italia); (c) trench excavation. Figures extracted from original documents.

The chosen technology was the traditional excavation (point 3 above) because it offered greater technical guarantees of non-interference with the structure of the adjacent and above the embankment. Both the micro-tunneling technique and the traditional tunnel excavation worried about possible phenomena of formation of voids in the structure adjacent to the tunneling or on its excavation front, with the creation of voids or cracks with triggering of possible filtering paths. Table 5 summarizes the technical and cost elements of the solutions evaluated: 
Table 5. Technical and cost elements of the solutions during the project stage.

\begin{tabular}{|c|c|c|c|c|}
\hline Technique & Penstock & Benefits & Drawbacks & Cost \\
\hline Micro-tunneling & $\begin{array}{l}2 \text { penstock DN } \\
2000 / 2500 \mathrm{PRFV} / \mathrm{ca} \\
+1 \text { concrete } \\
\text { channel for } \\
\text { discharge DN } 2000\end{array}$ & $\begin{array}{l}\text { Less interference } \\
\text { with above road }\end{array}$ & $\begin{array}{ll}\text { - } & \text { numerous perforations in } \\
\text { limited spaces } \\
\text { - } & \text { Excessive head losses } \\
\text { possible cavities between } \\
\text { ground and pipe } \\
\text { possible phenomena of } \\
\text { embankment lowering or } \\
\text { formation of privileged } \\
\text { filtration paths }\end{array}$ & $\begin{array}{l}910,000 \\
\text { euro/1,300,000 euro }\end{array}$ \\
\hline Traditional tunnel & $\begin{array}{l}1 \text { concrete penstock. } \\
\text { DN } 3600 \mathrm{~mm} \\
1 \text { concrete channel } \\
\text { for discharge DN } \\
2000\end{array}$ & $\begin{array}{l}\text { Less interference } \\
\text { with above road } \\
\text { Low head loss }\end{array}$ & $\begin{array}{l}\text { - Need to proceed with a } \\
\text { preliminary injections } \\
\text { consolidation of the above } \\
\text { bank structure } \\
\text { ground deformation in } \\
\text { front of the shield }\end{array}$ & $1,250,000$ euro \\
\hline Trench excavation & $\begin{array}{l}1 \text { concrete penstock. } \\
\text { DN } 3600 \mathrm{~mm} \\
1 \text { concrete } \\
\text { squared channel } \\
\text { for discharge } \\
2.5 \times 2.5 \mathrm{~m}\end{array}$ & $\begin{array}{l}\text { Low head loss } \\
\text { Reduced structural } \\
\text { and hydraulic } \\
\text { interference with } \\
\text { embankment }\end{array}$ & $\begin{array}{l}\text { Need to perform a top cut } \\
\text { of the embankment with } \\
\text { consequent involvement } \\
\text { of the road } \\
\text { interruption of vehicular } \\
\text { traffic and deviation of the } \\
\text { cycle and pedestrian one }\end{array}$ & $1,160,000$ euro \\
\hline
\end{tabular}

The design idea of a concrete pipe, instead of a steel one, made it possible to obtain a constructive advantage deriving from the lack of installation of the dielectric joint between the pipe and the bifurcated ones; this object should have been housed in an inspection well of important size and with significant costs. After identifying the technical solution in the trench excavation and concrete penstock, it became necessary to study the on-site building. A mobile formwork on wheels was designed alongside the technicians of the construction company (Parenti Costruzioni) and the technicians of the Peri company. They made it possible to create the pipeline in two semicircles, proceeding with a first phase of concrete casting (Figure 34), and then to reuse the same inverted formwork for the second phase casting of the upper cap of the penstock. The movement of the formwork up and down along the pipeline was assured through tracks. In Figure 35 the details of the work phases are depicted.

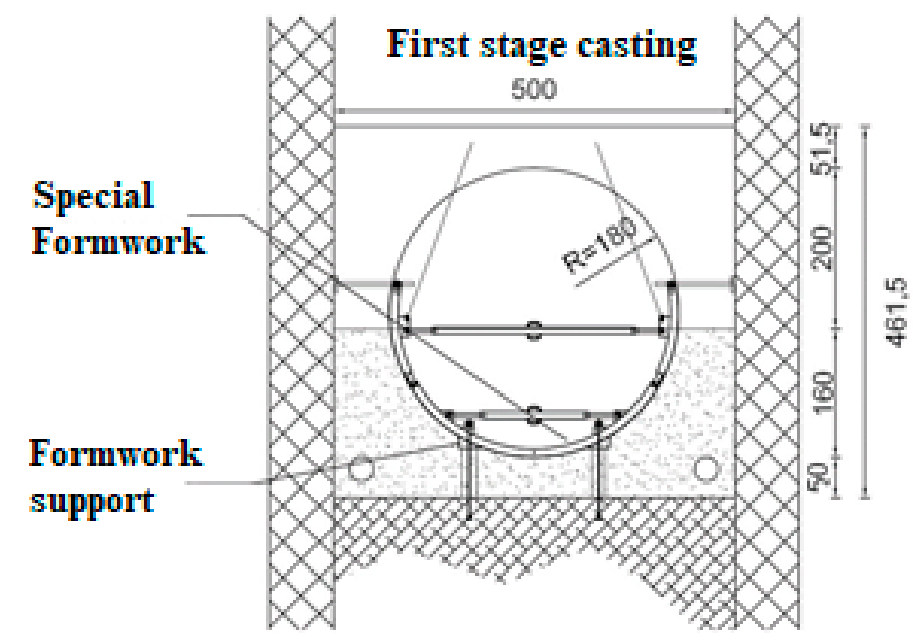

Figure 34. Mobile formwork was used for penstock building (courtesy Parenti Costruzioni and Peri). 


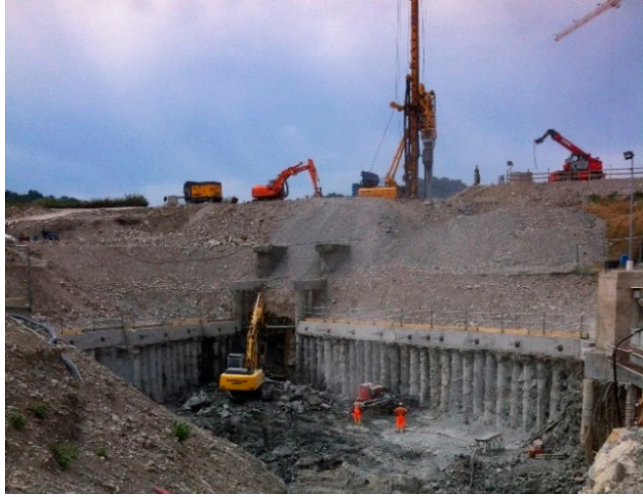

(a)

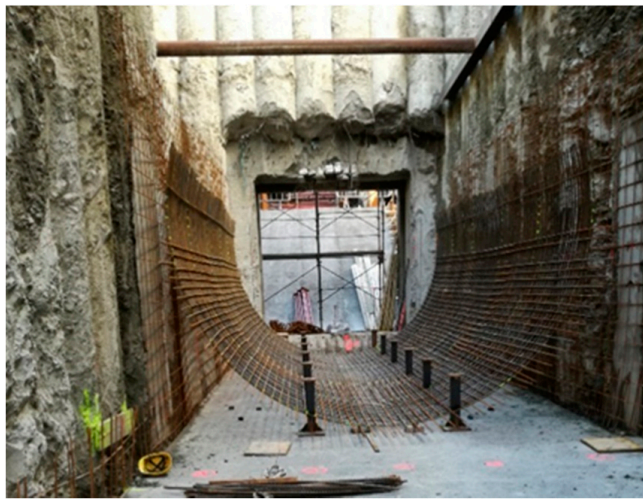

(c)

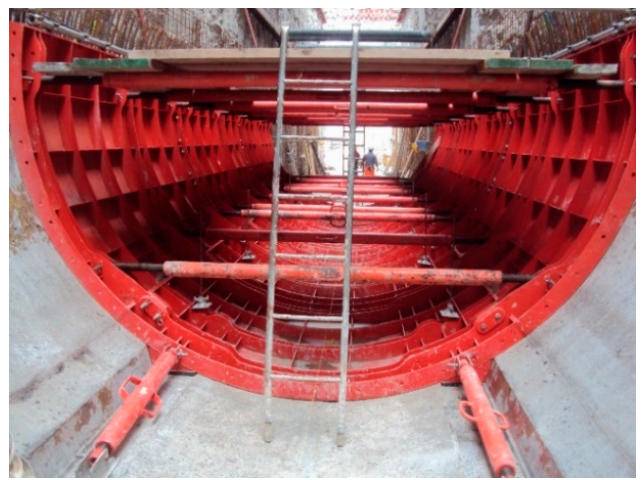

(e)

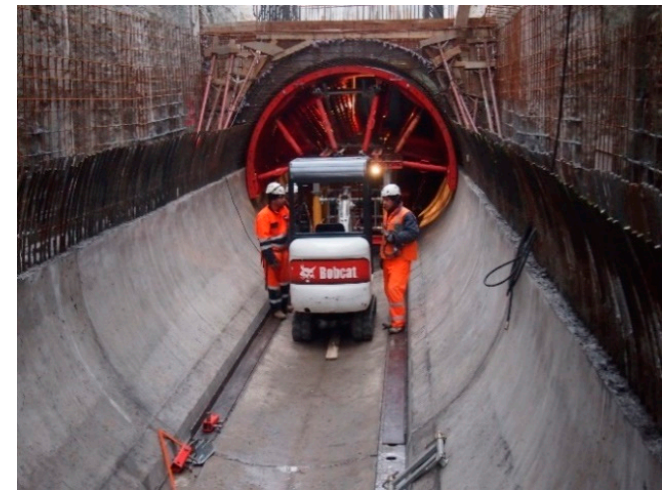

(g)

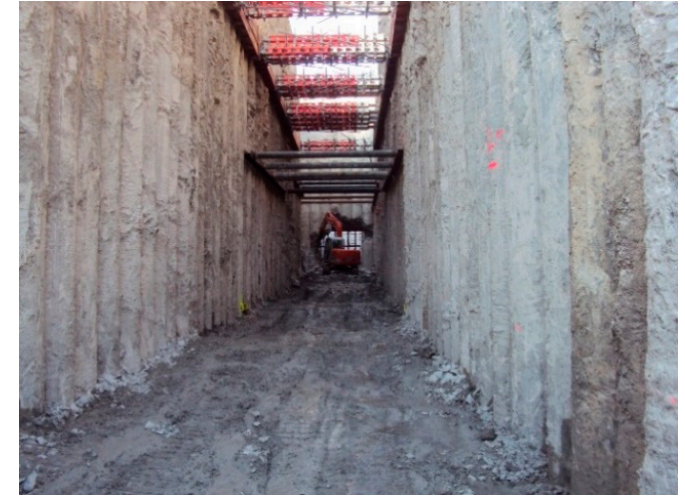

(b)

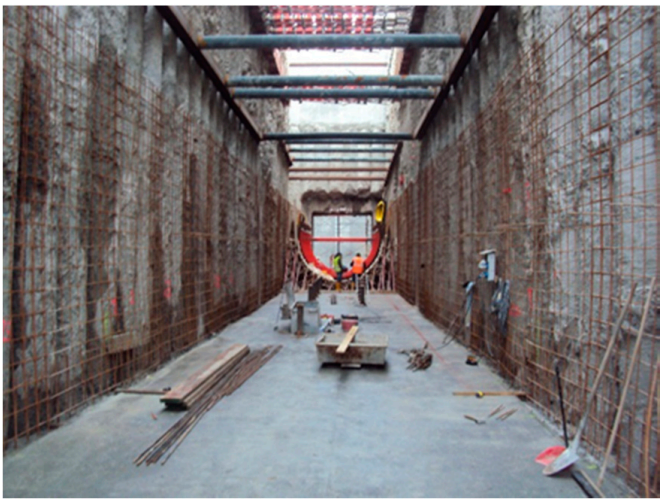

(d)

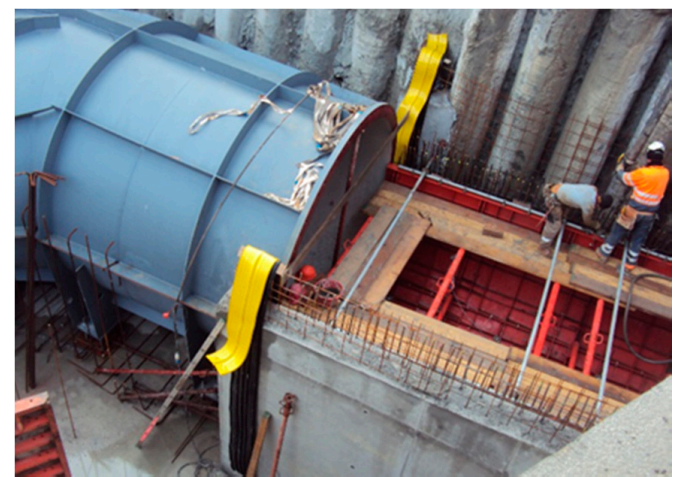

(f)

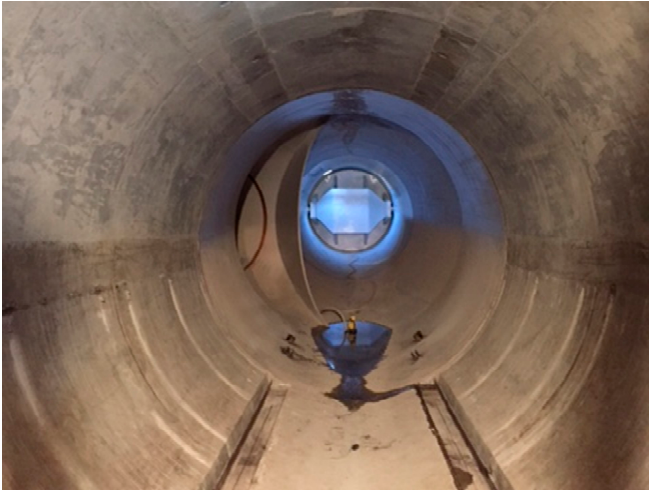

(h)

Figure 35. Working phases. (a) Building of secant poles; (b) support and excavation; (c) lower semicircle reinforcement; (d) formwork of the lower semicircle; (e) first phase of concrete casting; (f) joining of the penstock and bifurcation; (g) second phase of concrete casting; (h) penstock. 


\section{Mini Hydro on the Environmental Flow Overflow Structures}

The discharge necessary to preserve the river ecosystem, called environmental flow, could be used to produce green energy without any other type of pollution. A case of energy recovery from environmental flow through a mini hydropower plant is explained as follows.

The mini-hydropower plant is located in the North of Italy, in the alpine area of Predazzo close to Bellamonte, in the downstream part of Forte Buso dam. Primiero Energia SpA is the owner of the mini-hydropower plant, which is placed at the border of the Paneveggio-Pale di San Martino Nature Park. The main features of the Forte Buso plant are listed in Table 6.

Table 6. Geometric features of the Forte Buso plant.

\begin{tabular}{ccc}
\hline Geometric Feature & Units & Value \\
\hline Dam height & $\mathrm{m}$ & 105 \\
Maximum retention height & $\mathrm{m}$ & 99 \\
Maximum regulation elevation & $\mathrm{m}$ a.s.l. & 1458 \\
Minimum regulation elevation & m a.s.l. & 1395 \\
Basin storage volume & million $\mathrm{m}^{3}$ & 29.4 \\
Total head in maximum basin level $^{1}$ & $\mathrm{~m}$ & 114 \\
Total head in minimum basin level & $\mathrm{m}$ & 51 \\
\hline
\end{tabular}

${ }^{1}$ Total head for the turbine set at level 0 .

The hydrological basin has a catchment size of $60 \mathrm{~km}^{2}$ calculated in the dam's section. In addition, the other $38 \mathrm{~km}^{2}$ of downstream basins are linked with Forte Buso's basin to concentrate the environmental flow at a specific point. Along the downstream Travignolo river, the following values of environmental flow must be released:

- In winter (December-March): $392 \mathrm{~L} / \mathrm{s}$.

- In spring (April-July) and autumn (October-November): 549 L/s.

- In summer (August-September): $470.5 \mathrm{~L} / \mathrm{s}$.

The mini-hydropower plant is installed between the dam and the water point release in order to use the environmental flow to produce energy throughout the entire year. The large gap of head from the maximum and the minimum level of the lake (Table 1) is solved with the installation of a Pelton turbine connected with a Permanent Magnet Generator (PMG). This innovative solution is taken from wind turbine design which has to manage rated fluid speed problems.

The Pelton turbine has an installed power capacity of $580 \mathrm{~kW}$ with a vertical axis of rotation and has six jets with their security systems. The turbine speed is variable from 500 to 600 rotations per minute, the same as Permanent Magnet Generator, in accordance with the head variability. The PMG designed power of $600 \mathrm{~kW}$ is combined with a system of static energy conversion in order to connect the plant with the distribution network that works at $50 \mathrm{~Hz}$. The installation allows managing the Pelton turbine in the large variability of the total head without problems of cavitation that could damage the machine. The plant has a by-pass system that works also in case of electricity failure, to always ensure the correct release of environmental flow. The hydropower plant has an installed power capacity of $580 \mathrm{~kW}$ and a nominal designed power of $371 \mathrm{~kW}$ (medium annual value), able to produce 3,100,000 kWh per year of green energy (Figure 36).

In the last seven years (from 10th of August 2012 till the end of 2019) the mini-hydropower plant linked with the Forte Buso dam produced 22,131,138 kWh of green energy using the environmental flow that is released downstream of the dam. The new installation produced 3,019,000 kWh per year, as a medium value, which corresponds to a power production of $344 \mathrm{~kW}(3,019,000 \mathrm{kWh} / 24 \mathrm{~h}$ $\times 365$ days). This result explains a good working of the plant with a total medium yield of $92.7 \%$ compared to a traditional system (84-86\%).

The cost spent on the execution of the plant was approximately 2,086,000 $€$, while the overall cost consisted of $3,000,000 €$. Therefore $8150 € / \mathrm{kW}$ was the price for $1 \mathrm{~kW}$ of nominal power installed, 
which has led to a period of six years necessary to return the investment. If the current plant was not adopted it would have implied the use of two turbines, leading to an overall cost increase of $60 \%$, management and maintenance charges almost doubled, with the same total yield. The time required to return the investment would have been increased to nine years.

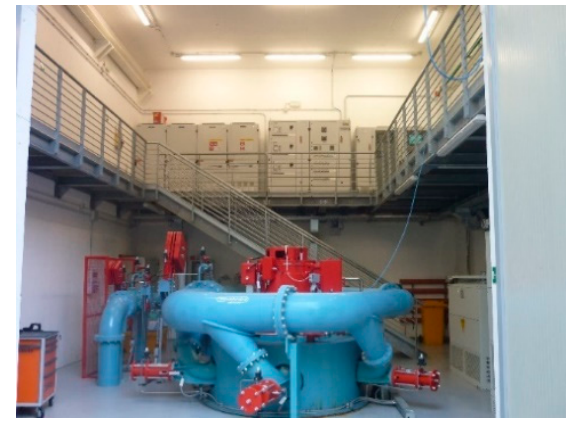

(a)

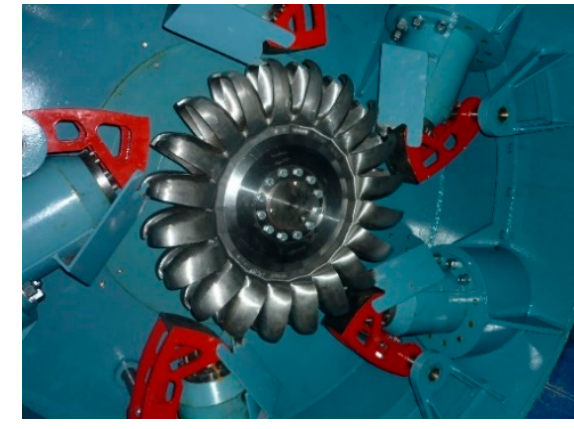

(b)

Figure 36. Mini-hydropower plant with Pelton turbine connected with Permanent Magnet Generator: (a) Picture of the entire hydropower plant; (b) Specific of Pelton turbine with the vertical axis of rotation and six jets.

One major challenge was to ensure the continuity of the environmental flow that required particular attention during the opening procedures of the by-pass system. This was solved by equipping the mini-hydropower plant with two alternative energy sources: a generator set and high-pressure oil-nitrogen iron tanks. Indeed in case of electricity failure, the two alternative energy sources ensure the correct operation of the by-pass system to always guarantee the release of the environmental flow in the Travignolo river. A needle opening valve of the by-pass system was adopted to dissipate the energy excess in the penstock to prevent any damage in the civil structures and the Travignolo river during the discharge operation (Figures 37 and 38).

The innovative designed solution adopted in mini-hydropower plants to use the environmental flow allowed saving 1905 tons of oil to produce the same amount of energy. Consequently, 5980 tons of carbon dioxide have not been discharged into the atmosphere. This methodology allows using the turbines out of their standard range of application with high yield.

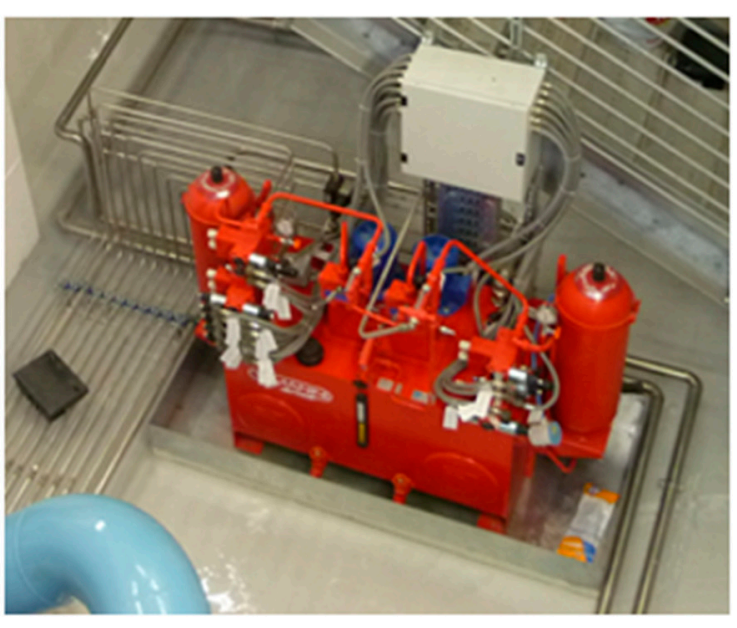

(a)

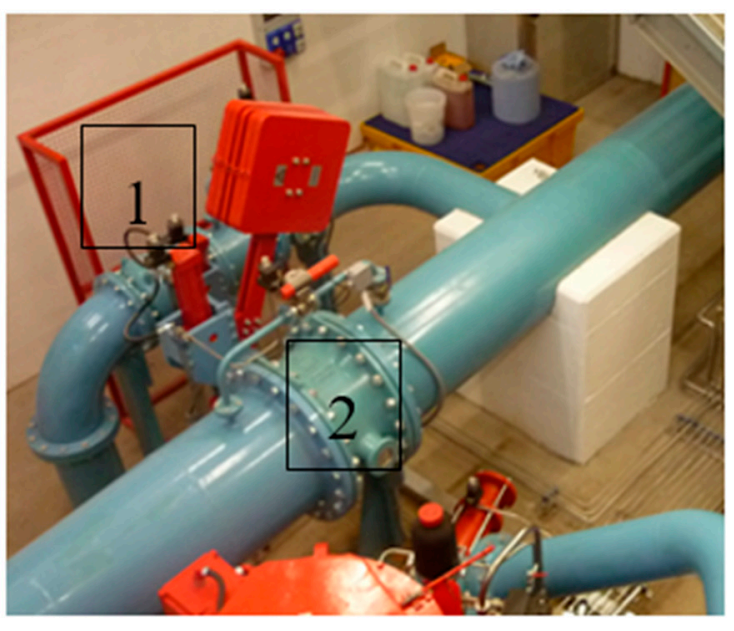

(b)

Figure 37. Additional hydraulic components in Forte Buso's mini-hydropower plant: (a) Picture of the high pressure oil-nitrogen iron tanks installed; (b) by-pass system and valves: (1) needle opening valve of the bypass system; (2) butterfly closing valve of the Pelton penstock. 


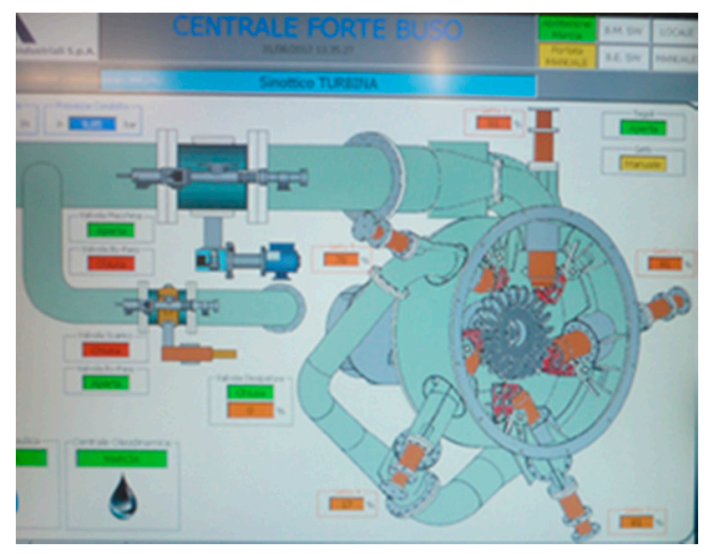

Figure 38. Scheme of the Pelton turbine, the penstock with its butterfly valve and the by-pass system with its needle valve.

\section{Susa Valley Water System and Energy Generation from the Water Network}

\subsection{Susa Valley Water System Description}

The Susa Valley Water System has been designed and built in order to supply water to a population of 115,000 inhabitants distributed in 20 municipalities that are at risk of suffering in times of drought. In fact, when a drought period occurs, the flow of water supplied by the springs, from which the single water systems are mainly fed, tends to decrease drastically. To overcome these problems, SMAT (Società Metropolitana Acque Torino), according to local and national authorities, decided to build a completely new water system using the water already available in the highest municipality of the valley, Bardonecchia. SMAT currently manages the integrated water services of 292 municipalities in the Turin Metropolitan Area, including those of Susa Valley (Italy), furnishing drinking water, sewage and wastewater treatment services to more than 2.2 million inhabitants. Among the major challenges that SMAT have to face is certainly the adoption of measures to mitigate climate change impacts, including: water loss reduction, increased resilience of water sources and creation of inter-municipal water networks by connecting multiple local water systems [52]. The case study here described represents one of the actions planned by SMAT to address the described challenges. Specifically this case study consists in the design and construction of three hydroelectric power plant powered by a new water supply system, the new Susa Valley Water System.

The Water System takes water from the 3,500,000 $\mathrm{m}^{3}$ artificial reservoir of Rochemolles Dam (1974 m a.s.1.), built between 1930-1935. This reservoir, currently used for hydroelectric purposes, stores water of high physiochemical quality for hydroelectric scope. As designed, the water released from the production plant (1260 $\mathrm{m}$ a.s.l.) supplies a new treatment plant located in Bardonecchia and it is distributed to all the 20 municipalities through the main pipe. The pipe is divided into two principal parts: the upper valley, characterized by steep slopes ( $2 \%$ on average, with $10 \%$ in the steepest profile) and the lower valley. The new hydroelectric plants are located in the first part.

As represented in Figure 1, the pipe, a DN700 ductile iron pipe, is divided into three sections:

- Bardonecchia-Deveys: $20.85 \mathrm{~km}$.

- Deveys-Chiomonte: $9.41 \mathrm{~km}$.

- Chiomonte-Gravere: $3.42 \mathrm{~km}$.

The inline tanks that maintain static water pressure below 25 or 40 bar are located at the end of each section. In order to reduce the pressure of inflow water and to produce electric energy, three Pelton turbines are installed. Therefore, the system has these peculiarities:

- the production plant is located at the end of a section fed by a reservoir.

- the water released by the turbines will be collected into another reservoir. 
- water discharge will be not constant along the pipe since are present connections with the water distribution networks of the municipalities.

- thanks to an agreement between the hydropower and the water supply companies, the water released from the hydroelectric power plant is constant during the week but varies in the range 250-500 1/s with a monthly timescale.

- it is necessary to guarantee the regular exercise of the system, also in case of production plant failure. Based on the above observations, the planned hydroelectric plants will differ from ordinary plants principally because they must be able to adapt to the different operating conditions of the water supply system. In particular, under ordinary conditions, they must operate in upstream reservoir level regulation but must be able to detect anomalous operating situations by temporarily switching to an adjustment on the downstream reservoir level in order to limit any possible waste of water caused by the maximum level being overcome. Moreover, plants will be equipped with a by-pass valve in order to guarantee pressure dissipation also in case of production plant out of order, allowing managing pipe flow rate carried out routinely by the regulation system.

A key characteristic of this system is that the size of the intermediate reservoirs is severely constrained by the topography of the valley. The smallest one, Deveys, has a capacity of $300 \mathrm{~m}^{3}$, but also Gravere, with a capacity of $1785 \mathrm{~m}^{3}$, considering a flow rate of $500 \mathrm{l} / \mathrm{s}$, can present level variation of $1 \mathrm{~m}$ in less than $120 \mathrm{~s}$. This entails difficulties in the management of the control devices (valves and turbines), which simultaneously have to keep water levels in reservoirs within the correct operating limits, optimize electricity production and avoid pressure transients. These aspects prompted SMAT to fund a study to investigate the problems related to the automation system of the control devices that will make use of a mathematical model created ad hoc [53] although over the last years several studies have focused on different aspects concerning the regulation of water supply systems using simulation models [54].

\subsection{Electric Power Productibility}

The production plant pre-sizing was carried out based on a management scenario in which the system provides, along the entire aqueduct line, the maximum flow rate withdrawn in Bardonecchia, equal to $500 \mathrm{l} / \mathrm{s}$, without taking into account withdrawals for the supply of local water networks. Table 7 shows the data used in the sizing of the production plants:

Table 7. Production plant nominal power estimation.

\begin{tabular}{ccccccccc}
\hline Plant & $\boldsymbol{Q}_{\max }$ & $\boldsymbol{Z}_{\boldsymbol{u}}$ & $\boldsymbol{Z}_{\boldsymbol{t}}$ & $\boldsymbol{H}_{\boldsymbol{g}}$ & $\boldsymbol{\Delta} \mathbf{h}$ & $\boldsymbol{H}_{\text {avail }}$ & $\boldsymbol{P}_{\max }$ & $\boldsymbol{P n}$ \\
\hline & {$[\mathrm{l} / \mathbf{s}]$} & {$[\mathrm{m}$ asl] } & {$[\mathrm{m}$ asl] } & {$[\mathrm{m}]$} & {$[\mathrm{m}]$} & {$[\mathrm{m}]$} & {$[\mathrm{kW}]$} & {$[\mathrm{kW}]$} \\
\hline Deveys & 500 & 1261.50 & 1012.00 & 251.50 & 38.90 & 212.60 & 885 & 950 \\
Chiomonte & 500 & 1010.00 & 744.60 & 265.40 & 16.90 & 248.50 & 1035 & 1000 \\
Gravere & 500 & 742.20 & 612.20 & 130.00 & 18.00 & 112.00 & 466 & 500 \\
\hline
\end{tabular}

where:

$Q_{\max }$ : maximum flow rate;

$Z_{u}$ : upstream altitude;

$Z_{t}$ : turbine wheel altitude;

$H g$ : altitude difference;

$\Delta \mathrm{h}$ : head loss at the maximum flow rate;

$H_{\text {avail }}$ : available head at maximum flow rate;

$P_{\max }$ : maximum electrical power, calculated with an efficiency of 0.85 ;

$P n$ : generator nominal power. 
The electric energy production expected has been estimated by implementing the following calculation procedure (using EPANET):

1. using the operating data provided for the water infrastructure design, the duration curve of the flow rates derived from the Bardonecchia intake has been calculated;

2. the delivery nodes along the line for the supply of the various municipal aqueducts have been identified with an assignment of the operating rules of operation of the branch;

3. the head losses in the individual sections of the pipe were estimated as a function of the flow rate determined as the difference between the flow rate derived from Bardonecchia and the flow rates subtracted along the line, by the Darcy-Weisbach equation;

4. the effective hydraulic head has been calculated on the production groups;

5. turbined flow estimated according to the operating rules assigned to the branches;

6. power and energy generated estimation in the reference period of the operating rule and determination of the annual production.

The following production scenarios were verified:

- $\quad$ scenario 1: in this scenario, the water system supplies the municipal distribution networks with the maximum flow;

- $\quad$ scenario 2: in this scenario, the water system supplies the municipal distribution networks with $50 \%$ of the maximum flow;

- scenario 3: represents a further refinement of the previous scenario; here the derivation of the maximum flow rates was considered in the periods of maximum water requirement and applying a $20 \%$ reduction in the other periods.

From the analysis of the above results, the overall average production expected from the ordinary operation will be in the order of $12 \div 13 \mathrm{GWh} /$ year. Table 8 collects the results of the simulations carried out.

Table 8. Estimation of annual production.

\begin{tabular}{ccccc}
\hline Scenery & Deveys & Chiomonte & Gravere & Total \\
\hline & {$[\mathbf{k W h}]$} & {$[\mathbf{k W h}]$} & {$[\mathbf{k W h}]$} & {$[\mathbf{k W h}]$} \\
\hline 1 & $4,094,465$ & $4,578,664$ & $2,238,775$ & $10,911,904$ \\
2 & $4,999,514$ & $5,674,226$ & $2,785,265$ & $13,459,005$ \\
3 & $4,706,430$ & $5,288,464$ & $2,588,769$ & $12,583,663$ \\
\hline
\end{tabular}

\subsection{Conclusions}

The new hydroelectric system here described is fed using water flowing into the main pipe of the new Susa Valley Water System. Turbines are located before the three inline tanks are used to reduce static water pressure. Since the Water System must integrate municipal water networks, especially in case of drought or low-quality water, the operating rules of the regulation system have to be a compromise between reliability and hydropower production.

\section{Implementation of a Multipurpose Project on an Irrigation Plant by Installation of an Innovative Counter Pressure Pelton Turbine}

\subsection{Introduction}

Hydropower is probably the most important renewable energy source in the Alps and it can be presumed that this will be the case for a long time ahead. Nevertheless, due to residual flow regulations and the required protection of water bodies new run-of-river hydropower plants on unexploited streams are hardly authorized. 
However, on existing water supply and irrigation networks a huge hidden hydropower potential can still be exploited. Due to its topographical relief, in the Alps water intakes at springs or streams often are situated at a much higher topographical level as the end-user, e.g., a drinking water supply network or an irrigation plant. This head difference represents a hydrostatic pressure that is frequently higher than the required operational pressure. In fact, in a drinking water supply network, an operational pressure of around 4 bar is usually established, whereas an irrigation plant equipped with sprinklers is operated with 3-10 bars. Consequently, if the hydrostatic pressure is higher than the requested operational pressure, this can be used to generate hydropower by installing a turbine on the main transport conduits and using the pressure in excess. In those cases, the used water body (spring or stream) does not undergo any consequences as the water is already captured for water supply or irrigation purposes. Of course, a hydropower plant on an existing network has to be economically feasible and must guarantee that there are no drawbacks for the primary use of the resource.

In the Vinschgau Valley, located in South Tyrol (North Italy), precipitation is very low. With mean annual precipitation of $500 \mathrm{~mm}$ the Vinschgau Valley is the most arid region in the Eastern Alps. For this reason, the agricultural areas of the valley since centuries are irrigated artificially. Originally the irrigation took place with the help of free-flowing superficial channels.

In the last 50 years, a huge number of artificial irrigation plants, equipped usually with sprinklers, have been built. These artificial irrigation plants very often cover hundreds of hectares. Due to the topographical traits of the valley, within one single plant, the head difference between the water intakes and the end-user can be up to $500 \mathrm{~m}$.

It is the case also for the irrigation plant "Malser Haide", which has been built in the years 2008 to 2012 (Figure 39). It covers 500 ha and the water intake of the plant is situated on a natural lake at a topographical height of $1448 \mathrm{~m}$, whereas the irrigated areas are located between 900 and $1300 \mathrm{~m}$. Thus, it is evident that the hydrostatic pressure within the plant partially is far too high. Therefore, in the plant three pressure interruption stations were provided. The first pressure interruption chamber is situated on the main transport conduit at a height of $1.265 \mathrm{~m}$. Since the commissioning of the irrigation plant, it was evident that the available hydrostatic pressure could be used for the generation of hydropower, instead of being abated by a pressure reducing valve followed by a pressure interruption chamber.

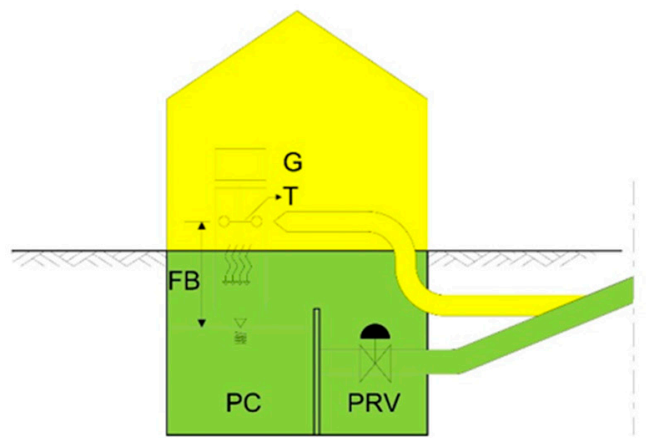

(a)

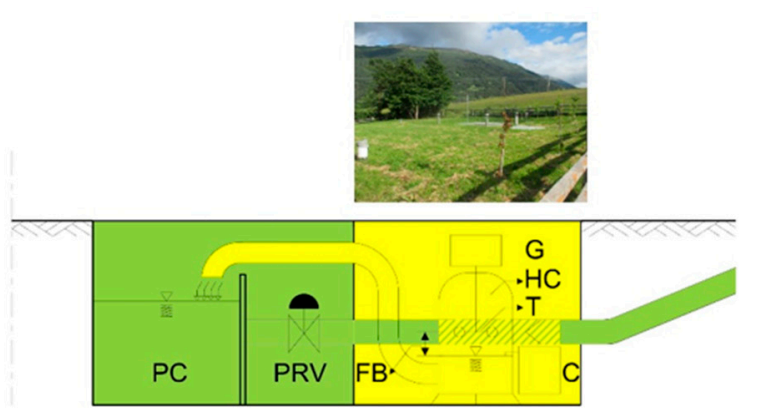

(b)

Figure 39. (a) Classic solution with the powerhouse above ground; (b) alternative solution with the powerhouse in the underground. Above the graph, a photo shows that on the surface only the steel cover (to take out the generator in case of revision) is visible. Symbols: $G=$ generator, $T=$ turbine, $\mathrm{FB}=$ freeboard, $\mathrm{PC}=$ pressure interruption chamber, $\mathrm{PRV}=$ pressure reducing valve, $\mathrm{HC}=$ hermetic case, $\mathrm{C}=$ compressor.

The owner of the plant started the development of a project for the implementation of a turbine that could transform the existing hydrostatic pressure into electrical energy. This paper describes the 
different project implementation steps, with the difficulties encountered and the innovative solutions that helped to overcome the difficulties.

\subsection{Project Enrolment}

\subsubsection{Feasibility Study and Technical Key Dates of the Hydropower Plant}

In a feasibility study, the general layout of the turbine with definition of the hydropower plant key data was studied (Table 9). The flow to be used by the turbine is driven by the demand of the irrigation plant. Depending on the area each single irrigation unit covers, the demand varies between 140 and 580 l/s. With the hydrostatic pressure being around 18 bars, a Pelton turbine, with at least four nozzles to cover the variable demand of the irrigation plant, resulted to be the best solution.

Table 9. Key data of the hydropower plant.

\begin{tabular}{cccc}
\hline Variable & Symbol & Unit & Value \\
\hline Gross head & $H$ & $\mathrm{~m}$ & 181.95 \\
Net head & $H$ & $\mathrm{~m}$ & 160.40 \\
Mean flow ${ }^{1}$ & $Q_{\text {mean }}$ & $1 / \mathrm{s}$ & 136 \\
Minimum turbine flow $^{2}$ & $Q_{\min }$ & $1 / \mathrm{s}$ & 140 \\
Maximum turbine flow & $Q_{\max }$ & $\mathrm{l} / \mathrm{s}$ & 580 \\
Gross capacity to be installed & $P$ & $\mathrm{~kW}$ & 825 \\
Yearly expected power output & $E$ & $\mathrm{GWh}$ & $\sim 1.1$ \\
Expected costs & $\mathrm{C}$ & $€$ & $1 \mathrm{Mio}$. \\
\hline
\end{tabular}

1 The mean flow is referred to the irrigation period lasting from 1st April to 31 st October, ${ }^{2}$ the minimum turbine flow is higher than the mean flow as the irrigation plant is only intermittently in operation.

\subsubsection{Authorization Phase}

After the feasibility study, the owner of the irrigation plant started with the authorization of the project. In the case of a classical Pelton turbine, between the turbine axis and the pressure interruption chamber, a height difference of at least $2 \mathrm{~m}$ would have been necessary in order to avoid submergence of the Pelton runner (Figure 39a). Consequently, a new powerhouse above the pressure interruption chamber would have been the solution.

As the site is situated in an area with strict landscape protection restrictions, where new buildings above ground are not allowed, the authorities rejected the presented solution. Thus, there was a need to look for an alternative solution. Different variants were evaluated in this context:

- To shift the powerhouse more upstream along the transport conduit, in order to gain the necessary height difference between the turbine axis and the water level in the pressure interruption chamber with the possibility to build the powerhouse underground;

- to install a reaction turbine (for example a Francis turbine);

- to install a classical Pelton turbine underground and to integrate it with a pump, able to cover the height difference between the tailwater level of the Pelton turbine and the water level of the pressure interruption chamber.

All these variants had some shortcomings. In the end, a request among several turbine manufacturers helped to identify the final solution. A counter-pressure Pelton turbine (key data in Table 10), where the tailwater is collected in a hermetic casing and then lifted to the pressure interruption chamber via a compressor, was chosen (Figure 39b). With this alternative solution, it was possible to erect the powerhouse in the underground. After having presented this solution to the authorities, the permission to build and operate the hydropower plant was delivered. 
Table 10. Key data of the turbine.

\begin{tabular}{|c|c|c|}
\hline Type & Unit & Value \\
\hline \multicolumn{3}{|c|}{ Pelton counter pressure turbine, vertical axis, 4 nozzles } \\
\hline Rotational speed & $\min ^{-1}$ & 750 \\
\hline Number of buckets & & 21 \\
\hline Diameter of runner & $\mathrm{mm}$ & 697 \\
\hline Width of bucket & $\mathrm{mm}$ & 184 \\
\hline Maximum efficiency of turbine & $\%$ & 91.5 \\
\hline Maximum electrical capacity & $\mathrm{kW}$ & 803 \\
\hline Installed capacity of generator & kVA & 873 \\
\hline Power need for compressor & $\mathrm{kW}$ & 22.6 \\
\hline Freeboard between turbine axis and tail water level & $\mathrm{mm}$ & 400 \\
\hline Diameter of casing & $\mathrm{mm}$ & 2200 \\
\hline Height of casing & $\mathrm{mm}$ & 2400 \\
\hline
\end{tabular}

\subsubsection{Implementation Phase}

In fall 2017, the new hydropower plant was constructed within four months. During the underground works, it was necessary to keep the irrigation in operation. Therefore, the hired contractor had the task to organize the construction site in a logistically sophisticated way. After the delivery and installation of the hydro-mechanical and hydro-electrical equipment, the plant could be commissioned and go into operation on 19th December 2017 (Figure 40). During the first two years of operation, some initial problems were encountered due to an under-dimensioning of the compressor. After having solved these problems, the plant is now running to the satisfaction of the plant owner. During the years 2018 and 2019, it could nevertheless produce 0.93 and $1.11 \mathrm{GWh}$, respectively.

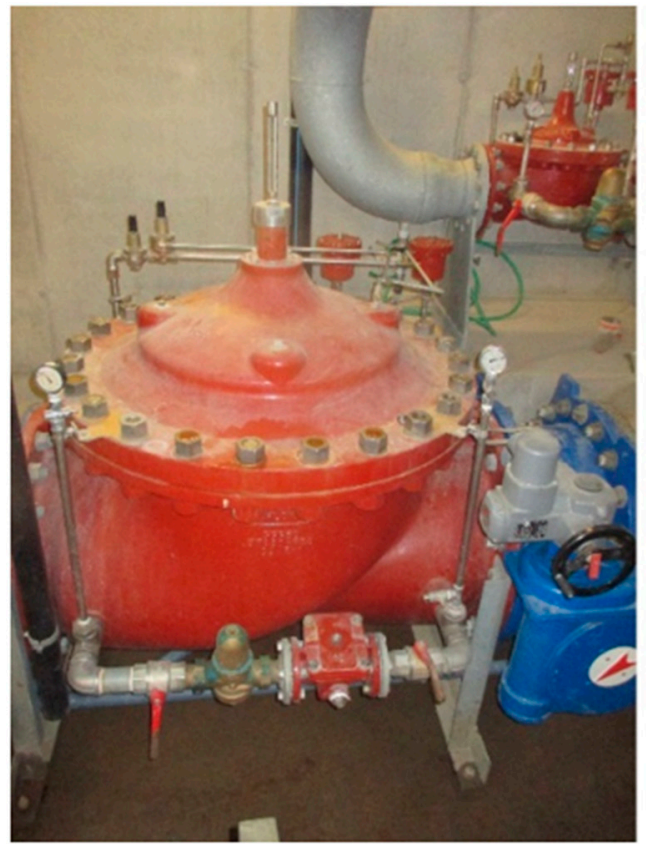

(a)

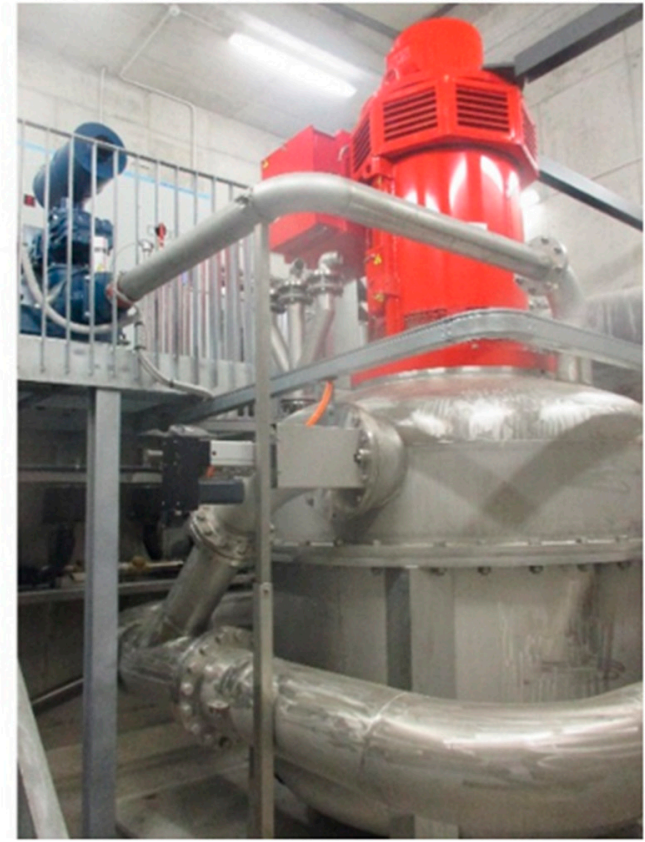

(b)

Figure 40. (a) Pressure reducing valve (in red) substituted by the new turbine. The pressure reducing valve was not removed as it will be needed in case of a turbine breakdown resp. revision; (b) new counter pressure Pelton turbine with turbine case (grey), generator (red) and compressor (blue). 


\subsection{Discussion on Innovative Aspects of the Installed Hydropower Plant}

\subsubsection{Counter Pressure Turbine}

The concept of the counter-pressure turbine was developed in recent years (Figure 41). For the manufacturer of the turbine, Häny AG from Switzerland, the turbine presented in this paper was a new record in terms of installed capacity.
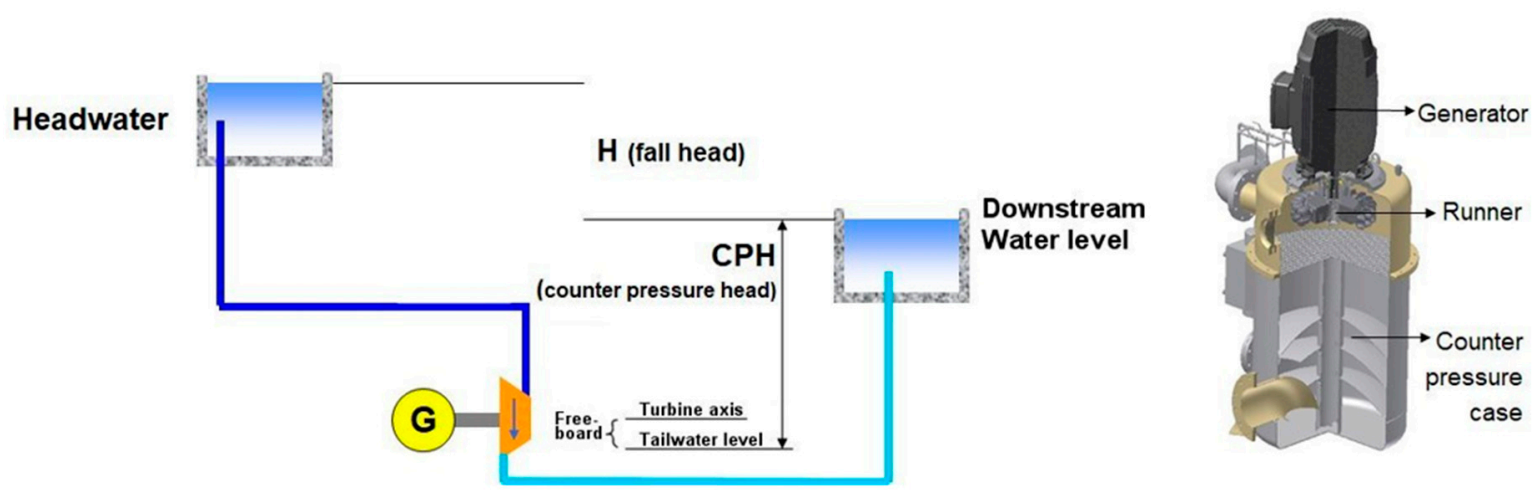

(a)

(b)

Figure 41. (a) Concept of counter-pressure turbine: the turbine axis is below the tailwater level with the turbined water being lifted to the tailwater level via a compressor; (b) counter pressure turbine with a hermetic turbine case. Between the turbine axis and the tailwater, a freeboard has to be guaranteed by the compressor which is exerting pressure on the water level in order to keep it at the established level.

The solution of a counter-pressure turbine is a very good possibility to install a turbine on existing water-supply networks, such as drinking water or irrigation plants, where valve chambers of water reservoirs already exist. The space needed by the turbine is limited, therefore frequently it is not necessary to carry out further civil works as the turbine can be installed in these existing chambers. The supplementary cost of the electromechanical equipment (hermetic casing, compressor system) is around $20 \%$ of the cost for a traditional Pelton turbine. However, the global cost of the plant is usually lower if no civil works to erect a powerhouse are needed.

In the present case, a height difference of around $5.5 \mathrm{~m}$ has to be overcome. Hence, the global fall head of the plant is reduced by this height difference. Moreover, the overall efficiency of the hydropower plant is slightly lower as also the compressor is an electromechanical machine with efficiency $<1$. Anyway, also adopting a classical solution, the available head is only represented by the height difference between the upstream water level and the water level of the pressure interruption chamber.

\subsubsection{Regulation of the Turbine}

Another innovative element is the regulation of the turbine. In high head run-of-river plants without impoundment, the turbine usually is regulated with the water level in the pressurizing chamber upstream. In this way, a turbine usually is working with the discharge diverted from the exploited stream. Consequently, the turbine power output is highly variable, being dependent on the available discharge of the stream.

In the present case, the regulation of the turbine has to follow the demand of the irrigation plant. The irrigation plant has sectors with variable areas that are irrigated, thus the demand for the irrigation plant can vary between 140 and $580 \mathrm{~L} / \mathrm{s}$. Due to its very limited storage volume, the pressure interruption chamber, situated immediately downstream of the turbine, cannot perform any function of balancing water demand and water availability. Therefore, the turbine has to work exactly with the discharge the irrigation plant instantaneously needs.

Thus, in the present case, a water level sensor was installed in the pressure interruption chamber and the turbine is regulated in order to keep the water level constant in this chamber. From one 
irrigation turn to the next the time delay is very short, as the demand can decrease during the closing stage of one sector in around $30 \mathrm{~s}$ to zero and then again increases in the same way when opening the next sector. The regulation of the turbine therefore has to be very smooth and reactive. In the first years of operation, the system had to be fine-tuned as in the first months of operation the regulation of the turbine was not yet perfect.

\subsubsection{Closing Law of Turbine Closing Valve}

Another innovative element in this plant is that on the closing valve of the turbine a bilinear closing law had to be implemented. In similar cases, the closing law is a single linear function. In the present case, during the first operational period, unexpected water hammer effects were observed. This phenomenon is critical for the pressure-reducing valve, still present in the system as this valve is fulfilling his original role while the turbine is turned off for revisions or inspections. Due to this undesired water hammer effects, the closing law of the valve had to be adopted. Now for the first $120 \mathrm{~s}$ the valve was closing quite fast, whereas in the last $30 \mathrm{~s}$ the closing was done in a very smooth way.

\subsection{Conclusions}

An irrigation plant was converted to a multi-purpose plant, through the installation of a counter pressure Pelton turbine, where previously a pressure reducing valve dissipated the present hydrostatic energy. Several innovative elements have been introduced in this plant. The plant certainly has to be seen as a benchmark example for multi-purpose use of water, as it uses the processed water both for hydropower generation and irrigation. Therefore ecological consequences on the exploited stream are absent as the water is already used for irrigation.

A counter-pressure turbine is a good technology to be installed in already existing systems. Not needing usually a new powerhouse, it allows reducing civil works to a minimum and therefore to optimize costs of investment.

\section{Variable Speed Application for Energy Recovery Hydropower in Aqueducts}

This case study describes an example of Energy Recovery Hydropower (ERH) in an aqueduct. ERH typically uses a hydro turbine to perform the function of a Pressure Reducing Valve (PRV): instead of dissipating the energy excess, the turbine converts it to clean energy, answering to both energy and ecology needs. This technology can be installed in a drinkable water distribution network and in general for manmade water-supply pipelines. Nowadays water manager authorities, municipalities and hydro turbines suppliers have created a consolidated framework synergy and a mature technology to retrofit existing Pressure Reduction Stations (PRS) with no further river modifications or land floods.

In this article the Solcano Small Hydro Power Plant (SHPP) case study is presented, exposing the advantages and main drawbacks of ERH and focusing on high-pressure variation and variable speed applications.

\subsection{Hydropower from Aqueducts}

The need to supply drinking water has forced the construction of hydraulic and civil works such as to be able to collect water from a specific water basin and make it available to end-users, as intake reservoir, tanks and pipelines; this environment is also ideal for hydroelectric plants.

Water distribution networks have been designed to meet the variable consumer demands keeping the pressure within an acceptable pressure range, to provide a reliable service. At the end of such long pipelines, PRVs are usually installed to reduce excessive pressure upstream distribution to end-users [55]. The pressure excess that had to be dissipated can be transformed from a problem to an income, with a double goal in economic and ecologic terms, replacing the valves with hydro generators.

Since 2000, there have been considerable technological developments in aqueducts "water-to-wire" turbine technologies: recent examples are the 4.6 MW aqueduct Hydro Power Plant (HPP) in the city of Athens, the Ranchos Penasquitos Pressure Control Hydroelectric Facility by San Diego Water County 
Authority with 4.5 MW generator installed [56]. In Italy, this technology is quite exploited in the North and Central regions. ZECO Hydropower S.r.l. has installed more than 30 aqueduct turbo-generators in Italy, Europe and South America.

Different advantages arise from this application: exploitation of available hydraulic energy potential, existing infrastructures, economic income, Green Certificates and renewable production. Revenue also depends on country incentive: for the present project, for example, the Italian legislation incentivizes the renewable production with feed-in tariff mechanisms, as 2009/28/EC Directive on drinking water quality has been transposed in by the Italian government with the Ministerial Decree 23 June 2016 "Incentivazione dell'energia elettrica prodotta da fonti rinnovabili diverse dal fotovoltaico" [56]. Additional benefits and difficulties are better discussed in [13,57].

\subsection{Solcano SHPP}

The Solcano SHPP is located in the province of Pescara (Abruzzo region, central Italy) in the municipality of San Valentino in Abruzzo Citeriore. The nominal diameter DN300 PN40 steel pipe feeds several municipalities as part of the "Acquedotto della Morgia" system with a maximum flow rate $Q_{\max }=0.185 \mathrm{~m}^{3} / \mathrm{s}$ flow rate. In the site of Solcano, the existing facility has the characteristics in Figure 42 , with very high head variation, as typical for aqueducts.
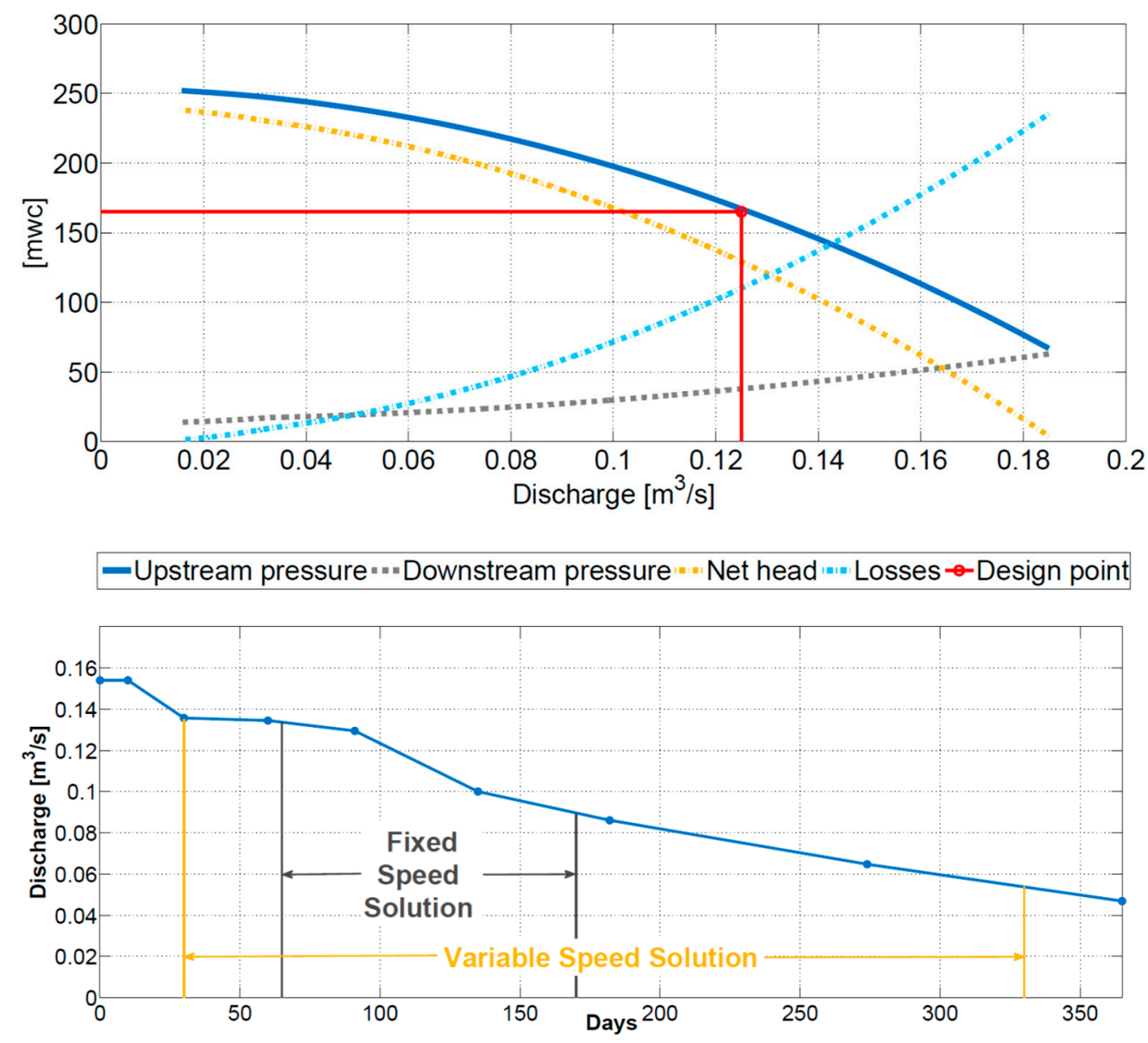

Figure 42. Solcano SHHP characteristics (top). As it can be seen, net head vary from $235 \mathrm{~m}$ up to $4 \mathrm{~m}$. Considering also flow duration curve a design discharge of $0.125 \mathrm{~m}^{3} / \mathrm{s}$ was chosen with an extra $10 \%$, for a $Q_{\max }=0.138 \mathrm{~m}^{3} / \mathrm{s}$ (left), with a design head $H$ of $132 \mathrm{~m}$. Solcano SHHP Flow Duration Curve (bottom).

As mentioned earlier, water flow in the aqueduct varies substantially due to the daily and hourly changes in water demand, therefore a proper turbine or turbine system must accurately be 
chosen [58,59]. $Q=0.125 \mathrm{~m}^{3} / \mathrm{s}$ was chosen also considering Flow Duration Curve (FDC), as seen in Figure 1; the site characteristic is suitable for Francis turbine with a speed $N=3000 \mathrm{rpm}$, in agreement with [60], but the wide head range made necessary a variable speed system. Faster synchronous speed is not possible and lower synchronous speed is not suitable for Francis turbines. Also, multi-stage Francis turbines increase dramatically the complexity of the system, lowering its reliability. The power plant load factor depends primarily on the turbine sizing so the analysis of the flow duration curve is fundamental, especially for aqueducts application, where head varies significantly with discharge.

The variable speed solution in this case (see Figure 42) increases the load factor; besides being a quite proven and reliable solution, the classic fixed speed Francis solution has a very narrow operating range with respect to site operating range. For Solcano SHPP reference diameter is $D=250 \mathrm{~mm}$ and speed varies between 2500 and $4000 \mathrm{rpm}$ to keep efficiency high.

Taking advantage of existing infrastructure, a variable speed Francis type solution with active flow control was designed and this flexible and reliable hydropower plant with virtually zero added environmental impact is capable of producing about $150 \mathrm{~kW}$. The aqueduct pressure surplus has been transformed into a source of profit (Figure 43).
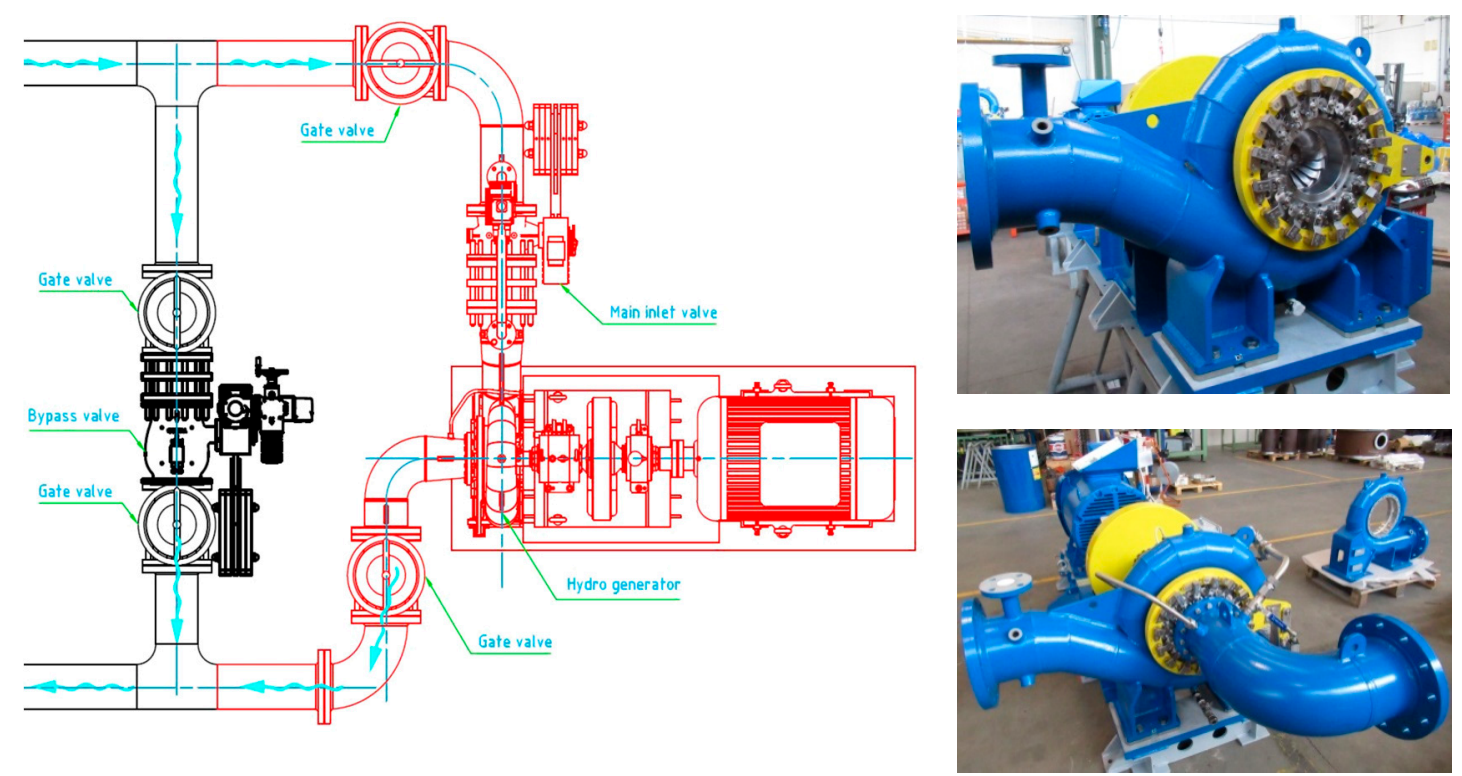

Figure 43. Solcano SHHP layout and model manufactured.

In this kind of application the speed variation and the high-speed values themselves pose not only a challenge to speed control itself but also to the mechanical design:

- High centrifugal forces at high-speed operation.

- High sliding speed for bearing, seals.

- Rotor dynamic calculation shall be performed to ensure resonance-free behavior over a wide range of operating speed.

\subsection{Drinkable Water Compliance}

The first priority of an aqueduct is not hydropower itself, but the reliable supply of drinkable water to consumers. So two main aspects must be considered when installing a turbine in an aqueduct: water drinkability and reliable water supply continuity.

\subsubsection{Drinkability}

Organoleptic characteristics of drinking water must be ensured with dedicated materials and paints for components in contact with water, satisfy specific regulations. The use of paints for food 
and beverage and stainless-steel components ensures the water to be returned to the aqueduct with unchanged chemical-physical characteristics. Material selection shall be in accordance with the country of installation local law.

In Italy, for the Solcano HPP project, components have to be designed in accordance to the Ministerial Decree 174/2004 "Regulation on materials and devices that can be used in fixed installations for capture, treatment, introduction and distribution of water intended for human consumption", transposition of the European Directive 98/83/EC on the quality of drinking water [2].

\subsubsection{Continuity}

One of the main challenges of such projects is of course reliability, as disturbances of the HPP and grid shall not affect water distribution.

The PRV is therefore not completely substituted by the turbo generator, but the original layout is modified with the parallel insertion of the turbine (Figure 43). When the turbine needs to be shut down for maintenance or in case of grid faults, the original flow path is restored, assuring consumers water supplying and keeping the hydropower production as a subordinate function of the aqueduct asset:

- When only the bypass is operating, the valve is commanded to open to release the flow required by the aqueduct operation.

- When the turbine is also generating power, the bypass can be closed by the quantity corresponding to keep the processed flow constant.

More information about the governing system is given in the next chapter.

\subsection{Governor and Transients}

Drinkable water systems are often subject to huge flow fluctuations (day/night, summer/winter) resulting in huge pressure fluctuations due to head losses in the pipelines, so that the higher the available flow, the lower the head and vice-versa.

The system hence has a double control system. The first acts on the inverter and regulates the machine speed according to a pressure-speed interpolation table defined in the design phase. The second governor system, this time global, regulates the flow required by the system and therefore acts by determining the operating elements (turbine, bypass or both including the limiter) to ensure the required flow and governing directly the bypass.

One of the major risks with conduit systems during manoeuvers is the integrity of the conduit itself. In case of ESD (Emergency Shut Down), the Francis turbine achieves maximum overspeed within seconds, with a runaway discharge reduced by $30-50 \%$ depending on specific speed [5], generating a water hammer condition; the resulting acceleration to runaway occurs in a few seconds considering the small inertia $\left(I=12.7 \mathrm{~kg} \mathrm{~m}^{2}\right)$. So, in order to keep the sum of both flows unchanged during the whole transient process (preserving the steady-state and hence lowering pressure wave intensity), the turbine is quickly closed and simultaneously the PRV is quickly opened, in a synchronous maneuver. This process also reduces the transient speed rise of the unit.

The bypass PRV is always equipped with a smart system customized to prevent excessive opening during transients that occurs at discharges lower than $Q_{\max }$; this feature avoids the valve to be opened more than required from users' demand and preserves pipe from buckling.

To check the ESD case, simulations must be performed including real turbine and valve characteristics as transients are highly sensible on the shape of these characteristics curves [59] (Figure 44). 


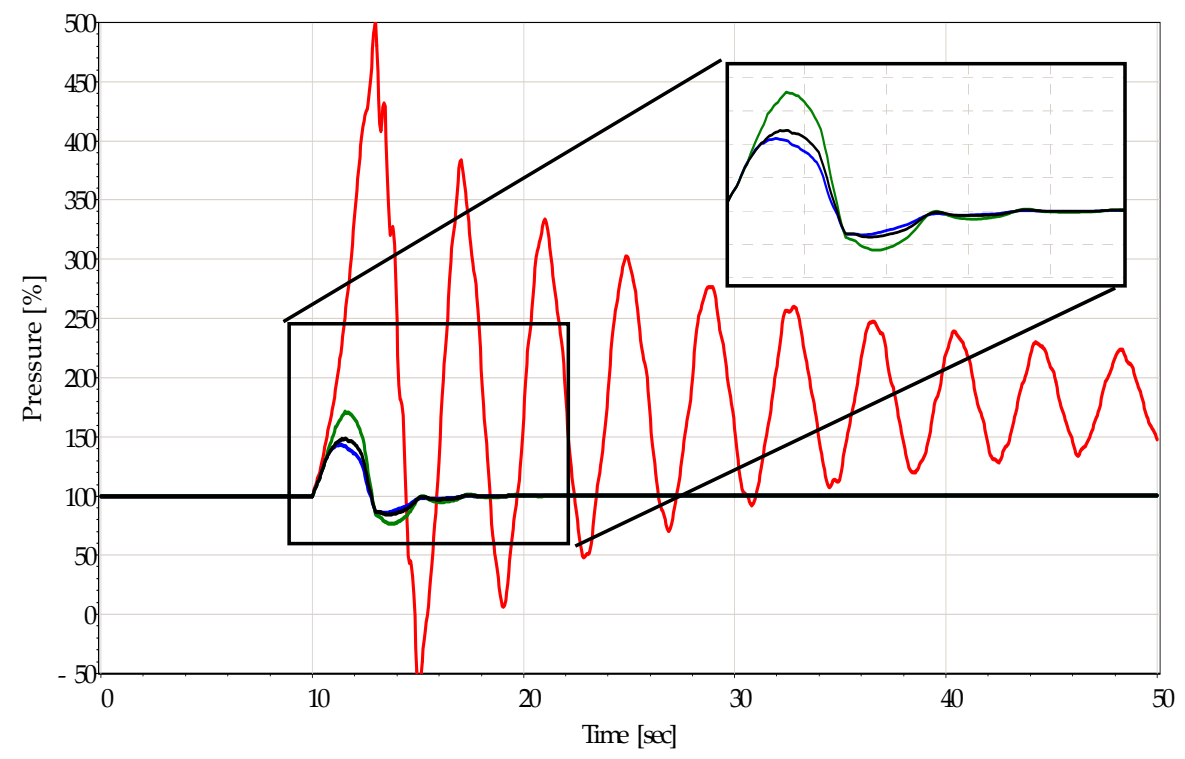

Figure 44. Non-dimensional (\%) pressure values for transient simulations with SIMSEN software for the Solcano project. Without a synchronous maneuver, the system is in danger (red line). With the synchronous opening of the bypass valve, overpressure can be reduced to $+75 \%$ up to $+40 \%$ (black-blue-green lines) depending on valve characteristics. An analysis with the valve manufacturer is fundamental. The blue line corresponds to quasi-linear valve characteristics, a solution chosen for the Solcano SHPP project.

\subsection{Conclusions}

High variable pressure drinkable systems are challenging for Conduit Hydropower:

- Highly variable power plant characteristics: variable speed.

- Water supply and quality: paints and coatings in accordance with regulation.

- The synchronous operation, with bypass branch. Sometimes, given the small size, it is not possible to install a large flywheel, and the characteristic times are very low. The maneuvers must be very rapid.

- The turbine synchronized with bypass through a smart system. Water availability and system safety to be assured.

- Given the small size, attention to returns and production of parts. Solid piece forging, $\mathrm{CNC}$ material to be preferred.

- Speed governor system required. Governing system complex, commissioning crucial for optimization.

\section{Study of Low-Head Hydrostatic Pressure Water Wheels for Harnessing Hydropower of Small Streams}

A renewed interest in hydroelectric plants of medium and small size below $2.5 \mathrm{~m}$ has been demonstrated by the scientific and international community in recent years (see, e.g., [61-63]), as these technologies are not only regarded as promising answers to cope with climate change impacts, but they could also unlock a huge hydropower potential, currently unexploited. Considering European countries only, the estimated available sites account for $13 \mathrm{GW}$ of untapped hydraulic energy potential [62]. Various concepts of small hydraulic turbines are under research and optimization process, and the "Hydrostatic Pressure Machine" (HPM, Figure 45) is the one considered in the present study [63], operating on sites with ultra-low hydraulic heads between 0.5 and $2 \mathrm{~m}$. Due to its design, the HPM converts the hydrostatic head created by the turbine itself: the difference between the upstream and downstream water levels applies a net pressure force on the machine blade in the same direction as the 
water flow, resulting in an applied torque on the machine shaft, thus converting hydraulic power into mechanical power [63-65]. The HPM is not only an energy converter, but it can also be used as a weir to regulate water levels in any river and irrigational canal.
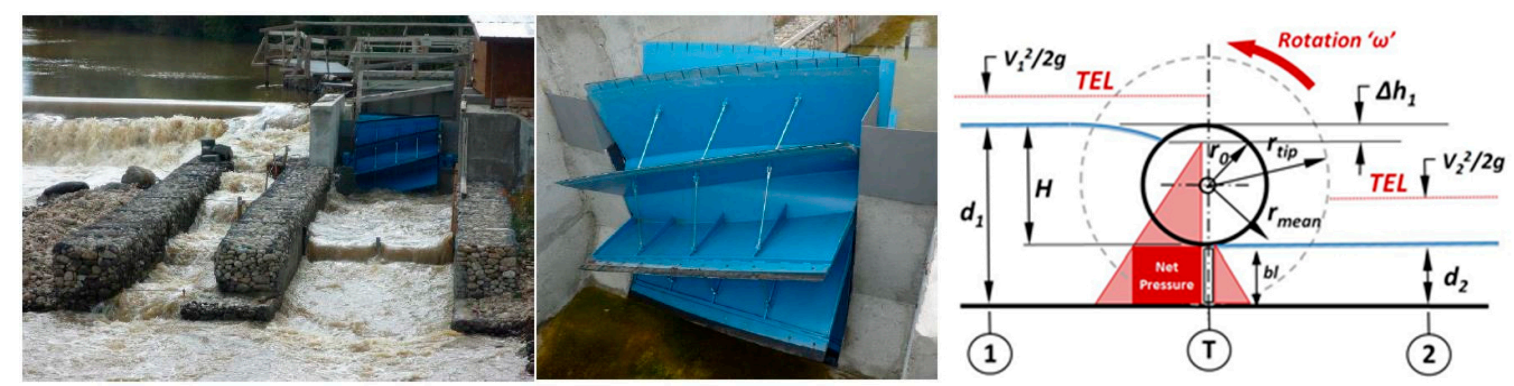

Figure 45. Hydrostatic Pressure Machine (HPM) under real field trial, developed by the HYLOW project, Bulgary (reprinted from [61]), and 2D scheme of an HPM under nominal operating conditions.

\subsection{Experimental Set-Up and Results}

Two different small-scale models of HPM have been tested in the "Herode Channel", a pre-existing experimental facility located in the research institute IRPHE, in Marseille. The two experimental scale models have different geometries and they were designed to study the HPM under various operating conditions.

\subsubsection{The Herode Hydraulic Channel}

In Figure 46, a schematic view of the Herode channel is given. This testing facility is divided into two main areas: the free-surface zone with a rectangular cross-section, that corresponds to the actual testing environment and the recirculation pipe located below. In the testing section, the free-surface level can reach up to $0.55 \mathrm{~m}$ and the internal width of the channel is $0.62 \mathrm{~m}$, while the total length of the testing section is $8 \mathrm{~m}$.

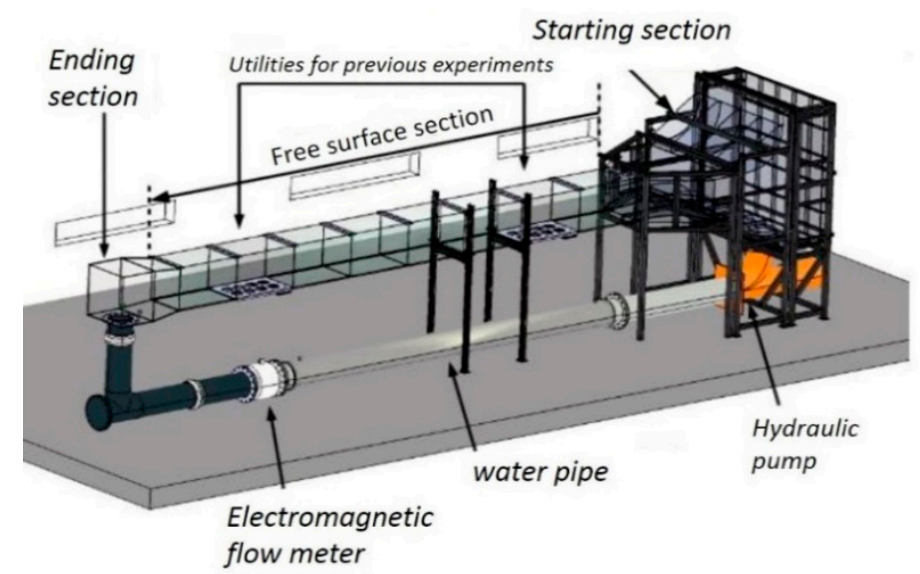

Figure 46. Schematic view of the Herode channel at IRPHE with a testing section of $8 \mathrm{~m}$.

\subsubsection{PYTHEAS Technology's Wheel}

For the PYTHEAS Technology experimental HPM model, two parts were designed: the HPM wheel and a shroud. The hydraulic wheel is designed with 12 blades attached to a central hub. Blades are as long as the diameter of the central hub. To minimize turbulence each time a blade enters the free surface, they present an entry angle of $30^{\circ}$, and the twisted shape not only improves the blade cell emptying but also prevents air to be sucked in at the entrance. The second main element of the system is the shroud. It consists of an adaptation of the channel floor to ensure efficient compression 
during the wheel cycle and to reduce the by-passed flow underneath the wheel blades, as depicted in Figure 3. Various shroud configurations were tested: the covered angle between the vertical and the entrance or exit section could be $40^{\circ}$ or $56^{\circ}$, as shown in Figure 47 . To optimize the filling and emptying of the cells, the wheel should be smaller than the complete inner width of the channel where the HPM is installed. According to [66], the gap between the wheel sides and the channel lateral walls must be at least equal to half of the wheel's width, especially on the downstream part where the water needs to be quickly discharged. This is the reason why the wheel axial length is $238 \mathrm{~mm}$, the blade radial length is $150 \mathrm{~mm}$ while the hub diameter is $150 \mathrm{~mm}$ (Figure 48). More studies about the impact of the gap between the wheel sides and the channel lateral walls have been made by Butera et al., 2019 [67].

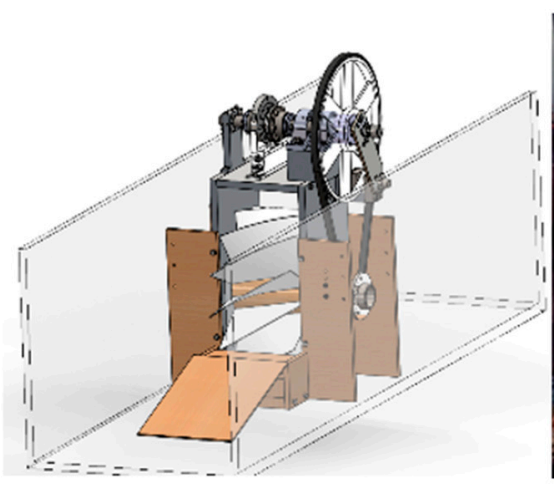

(a)

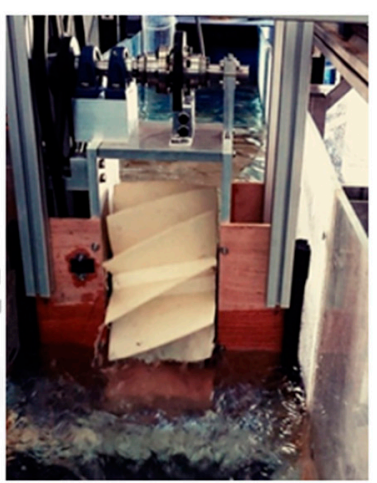

(b)

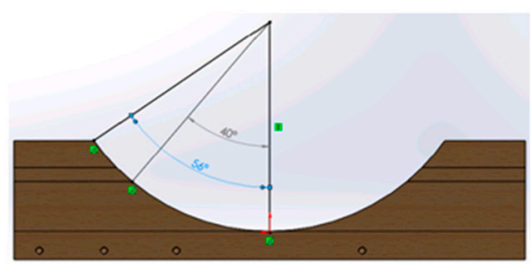

(c)

Figure 47. Model views of the PYTHEAS experimental system ((a): CAD (Computer Aided Design) drawing of the wheel; (b): picture of the wheel operating in the flume; $(\mathbf{c})$ : shroud design of PYTHEAS HPM scale model).

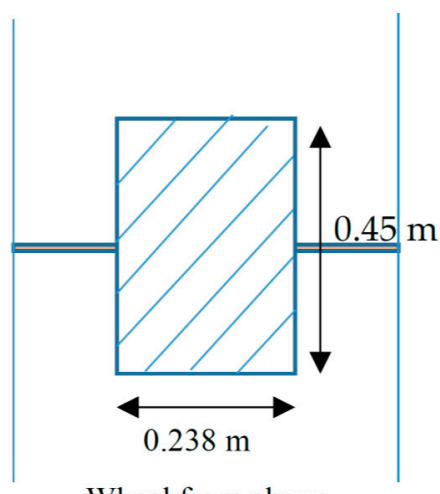

Wheel from above
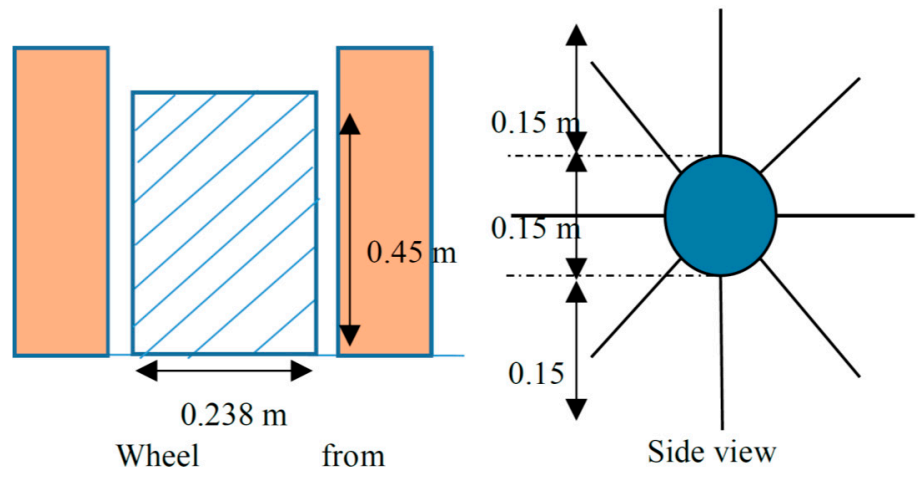

Figure 48. Scheme of the tested wheel.

Rotational speed and torque are measured by a torque sensor with an accuracy of $+/-0.1 \%$ and an encoder speed with an accuracy of $0.5 \%$ on a shaft above the free surface. The resistive torque is applied with a mechanical brake, manually adjustable thanks to two plates tightened by screws on the transmission shaft. For the experimental protocol adopted for the PYTHEAS experiments, it must be noted that the testing channel operates as a closed-loop. At the beginning of the tests, with the pump running, the operator sets the upstream water level at the chosen nominal value of $31.5 \mathrm{~cm}$ while, at the same time, the resistive torque is set at its maximum. Then, the operator increases the pump power and reduces the braking torque to keep the upstream water level constant and record data for various flow rates and mechanical load conditions. Given the initial presence of enough water in the channel, it was possible to fix the downstream water level at the nominal value of $16.7 \mathrm{~cm}$. 
The nominal head difference is then $H=14.8 \mathrm{~cm}$ and is constant all along the studied power curve conditions. The diameter of the HPM is $45 \mathrm{~cm}$, and the width is $23.8 \mathrm{~cm}$ (Figure 48).

\subsection{Results}

Figure 49 shows the power curves and efficiency obtained with two shroud conditions. The red curve corresponds to a $40^{\circ}$ downstream shroud, the blue one is set with a downstream shroud angle of $56^{\circ}$, while the upstream shroud angle is kept constant at $56^{\circ}$ for both tests. The gap between the two curves can be explained by the ventilation phenomenon, when the blade gets out of the water. With the tallest shroud, the water remains in the cell for a longer time: an effort from the wheel is then required to rise this volume before air fills it, creating additional losses.
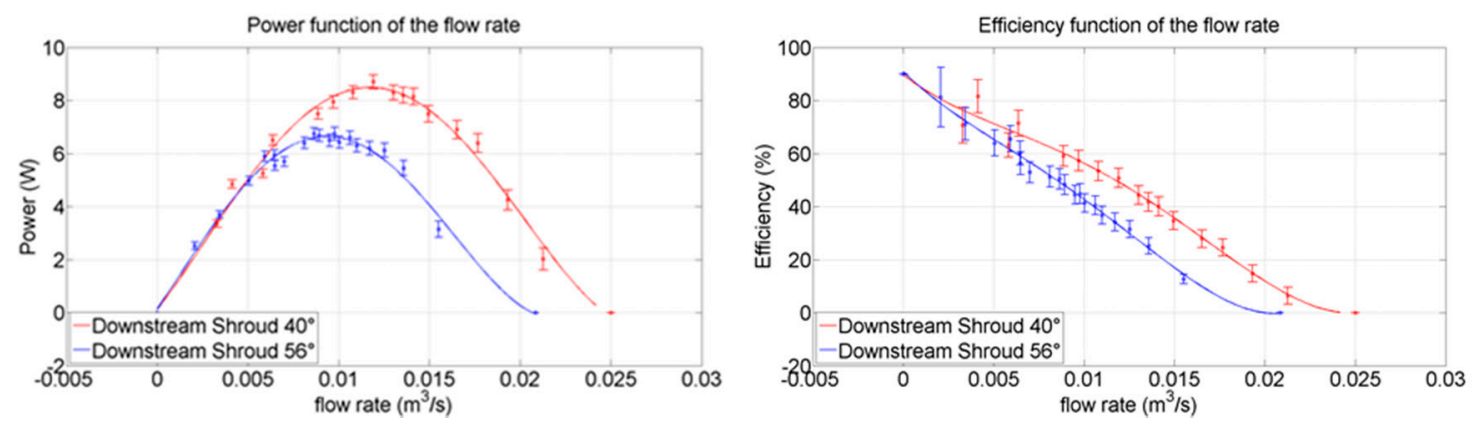

Figure 49. Converted power for two downstream shroud configurations (PYTHEAS wheel).

Finally, Figure 50 shows the difference between the analytical model developed by Linton. Some experimental coefficients were adjusted to the PYTHEAS wheel. The left figure shows the flow rate as a function of the rotational velocity. As expected, the flow rate through the turbine is nearly linear with its rotation speed. The right figure shows the power and efficiency of the analytical model and experiments (with error bar).
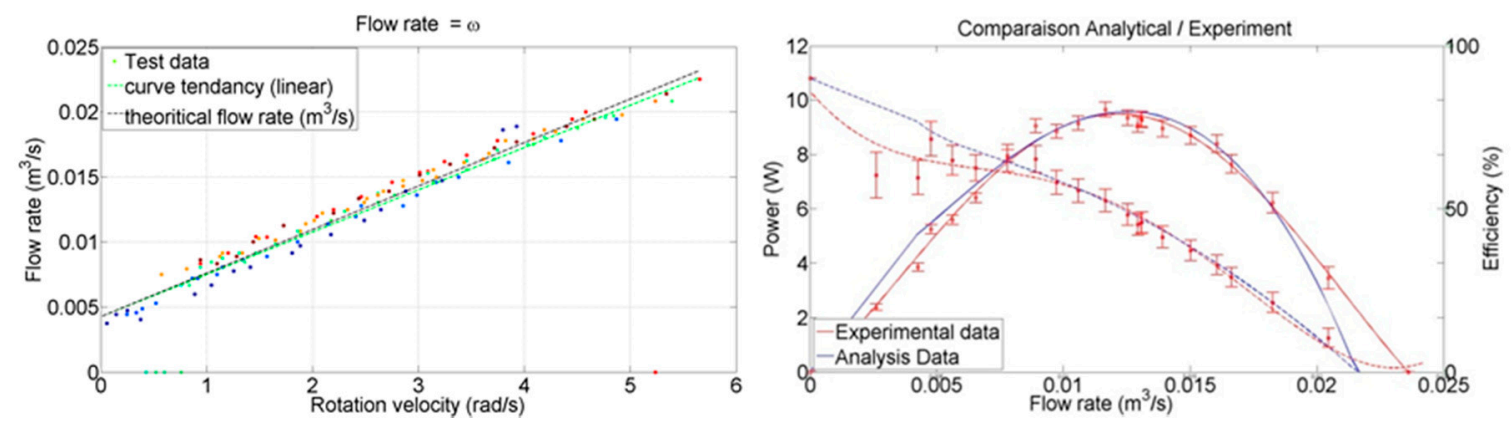

Figure 50. Variation of flow rate as a function of the rotation velocity (PYTHEAS wheel).

It is possible to compare these results to those found by Butera et al. (2019), reference [67], who found an optimal $N^{*}=\frac{v}{\sqrt{2 \cdot \mathbf{g} \cdot \mathbf{H}}}=0.2$, where $v$ is the tangential wheel speed, and $H=0.148 \mathrm{~m}$ is the head difference. In the PYTHEAS case, according to Figure $6, N^{*}=0.33$, with $v$ the optimal tangential speed, i.e., the wheel speed at the optimal flow rate $Q_{o p t}=0.012 \mathrm{~m}^{3} / \mathrm{s}$. The higher rotational speed confirms the fact that an optimal shroud design allows the wheel to rotate faster, thus reducing power losses and maintaining efficiency (at the optimal flow rate point) of slightly less than $60 \%$, in agreement with literature.

\subsection{Conclusions and Perspectives}

The tests performed in this study show a very good agreement between the analytical model and the experimental results. Based on these reliable results, power production and efficiency for a 
given site of any scale can be estimated. At the optimal operating point in flow rate, or equivalently in rotation speed, the HPM efficiency is slightly less than $60 \%$. This type of turbine appears as one of the most suitable solutions to efficiently harvest energy from straight channels with no head drops, where small head differences can be generated.

The results considering the angle of the shroud matched the ones expected and losses were quantified when other shroud angles were used. Only a few tests have been done for the HPM, thus the experiments conducted by PYTHEAS Technology were necessary to verify the literature analytical and CFD model. Furthermore, in order to ensure that the flow rate control is possible by controlling the HPM's rotational speed, the linear law showed in Figure 50 needed to be validated.

The next steps of the project will address: (i) the adaptation of the generator that converts the slow motion of the wheel into electricity, and (ii) the elaboration of the control law of the wheel. Another advantage of this wheel is indeed the linear relationship between flow rate and rotational speed. This relation makes the wheel also useful for flow rate control. By adjusting the rotational speed of the wheel, one can control the flow rate and produce power simultaneously: this is no less than a hydraulic regulation work harvesting energy.

\section{The Mariucci Turbine as Optimization of the Girard Turbine}

The Mariucci turbine is a new action turbine, patented in 2017 (Patent N. 0000282352) thus a hydraulic turbine that exploits the kinetic energy of a water jet. It is an optimization of the Girard turbine, improved to reduce leakages and with a better regulating system for efficiently working at partial flow conditions [68]. The Mariucci turbine is an axial flow machine since the water jet flows parallel to the vertical rotation axis of the turbine. Existing action turbines (Pelton and Turgo turbines, [69]) in very low head sites (e.g., below $3 \mathrm{~m}$ ) can exploit very small flow rates (few tens of liters per second), thus generating very low power output, while the Mariucci turbine, being an axial turbine, can exploit flow rates up to $6 \mathrm{~m}^{3} / \mathrm{s}$. Furthermore, being an action turbine, it is less expensive than Francis and Kaplan turbines. The machine operates in a wide range of $n_{Q}=n *\left[\left(Q^{1 / 2}\right) /\left(H^{3 / 4}\right)\right]=6 \div 80$, with flow rates $Q$ ranging from 0.3 to $6 \mathrm{~m}^{3} / \mathrm{s}$, head differences $H$ within $1.5 \div 8 \mathrm{~m}$, and rotational speeds of $30 \div 100 \mathrm{rpm}$.

\section{Turbine Description: Working Principle, Flow Regulation and Efficiency}

The turbine system is composed of a distributor, developed as a circular crown, above the turbine, where the water flows axially. The entire machine is adjustable in height by means of hydraulic pistons as a function of the water level downstream, in order to reduce the unexploited head at the outlet (Figure 51). The impeller rotates in the atmospheric environment, avoiding cavitation.

An impeller blade (Figure 52a) is composed of a central surface where water flows, and an external wing and an internal wing with upward development, to prevent the tangential volumetric losses from the gap between the distributor and the impeller. The blades are welded to an internal circular shroud and two external bands, a circular one and a conical trunk.

The absolute speed vector of the water entering the nozzles $v_{11}$ forms an angle $\alpha^{\circ}$ with respect to a horizontal plane, while at the outlet the absolute speed vector $v_{12}$ forms an angle $\beta^{\circ}$ (Figure 52b). At the entrance of the impeller, the inclination of the blades, (angle $\gamma^{\circ}$ ), is given by the direction of the speed vector $w_{21}$. The speed $w_{21}$ is given by the vector difference between the absolute speed $v_{21}$ and the tangential speed $u$ (Figure 52). The ratio between $u$ (at the median radius) and the absolute flow speed $v_{12}$ is $|u| /\left|v_{12}\right|=0.46 \div 0.50$. The absolute outlet speed $v_{22}$ from the impeller is designed to be vertical by fixing the inclination of the blades at the trailing edge, by fixing the outlet blade angle $\delta^{\circ}$, i.e., the inclination of the relative speed $w_{22}$. The absolute speed $v_{22}$ is the vector sum of the relative speed $w_{22}$ and the drag speed $u$ (Figure 52). 


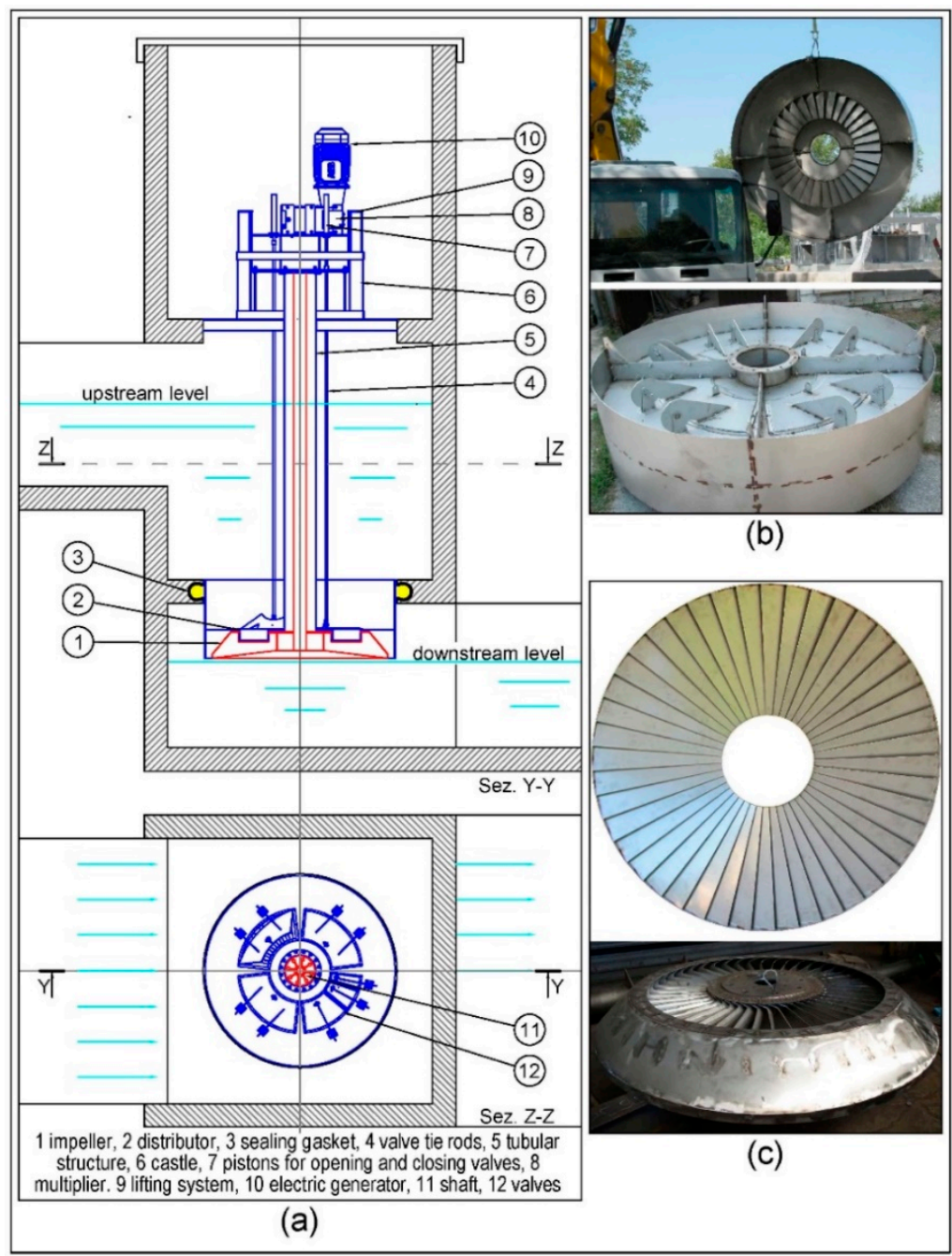

Figure 51. Sketch and real pictures of the installation: (a) turbine in a tank system. (b) Photo distributor: the water flow can be partialized ( 28 jets). The closing and opening of the nozzles take place by means of valves. (c) Photo impeller: the blades have a shape that reduces volumetric losses to zero.

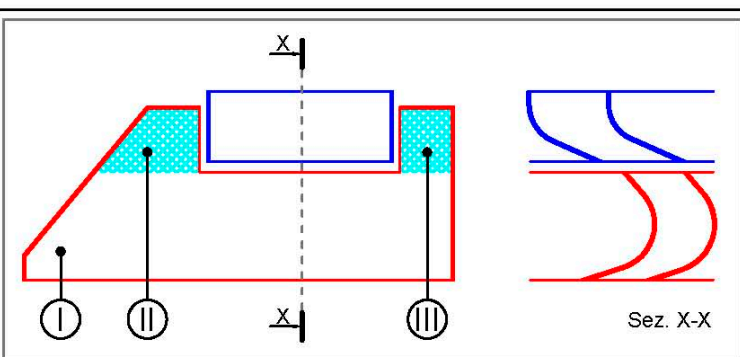

(a)

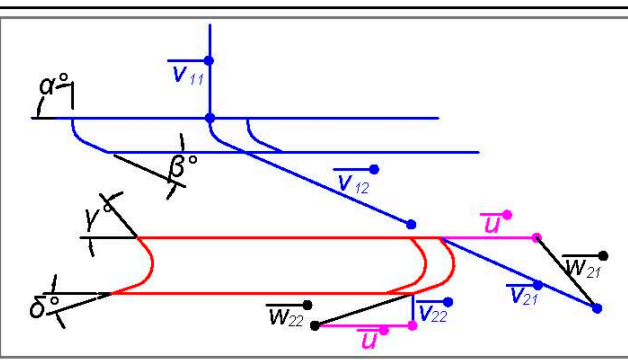

(b)

Figure 52. (a) Schematic representation of the turbine (Impeller blade(I), External wing (II), Internal wing (III)) and (b) velocity triangles of the section X-X.

The regulation of the flow rate is provided by four nozzles distributed on the circular crown above the distributor. Each nozzle covers a circular sector of $90^{\circ}$, i.e., seven stay vanes. Three of them are on/off, so that they can allow/avoid the flow passage through seven stay vanes simultaneously. One nozzle, instead, can be further regulated in order to allow/avoid the flow through one stay vane 
passage each time, thus gradually passing from the off position to the complete opening (seven stay vanes) at one stay vane passage step. This nozzle is used both at low flow rates, but also to gradually pass from $n$ open nozzle to $n+1$ open nozzle, with $n$ from 0 to 3 . The closing or opening of some nozzles does not alter the kinematics and dynamics of the other flows, therefore the hydraulic efficiency remains constant for each flow. The on/off valves allow to vary the flow rate by $1 / 28$ of the maximum value (Figures 53 and 54a).

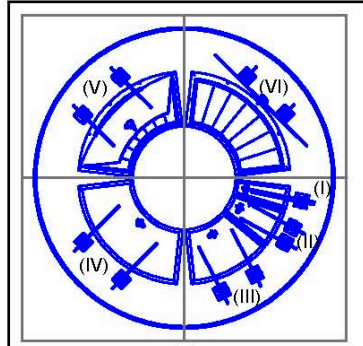

(a)

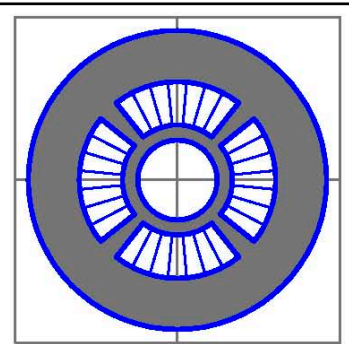

(b)

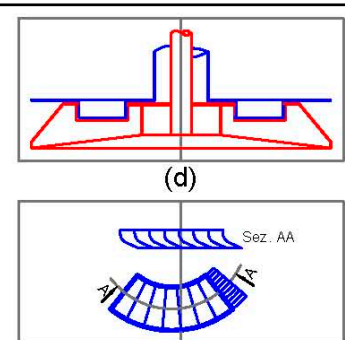

(c)

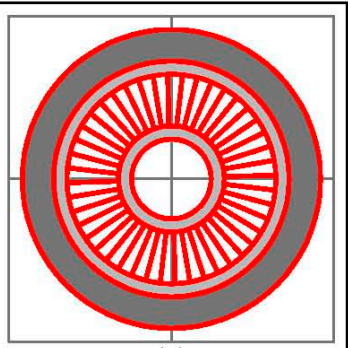

(e)

Figure 53. Distributor and impeller: (a) Nozzle: (I), (II), (III), (IV) closed; (V) on opening; (VI) open. (b) Distributor floor plan. (c) Plan and section of a sector with seven nozzles. (d) Joint distributor and impeller section. (e) Planimetry of the rotating impeller.

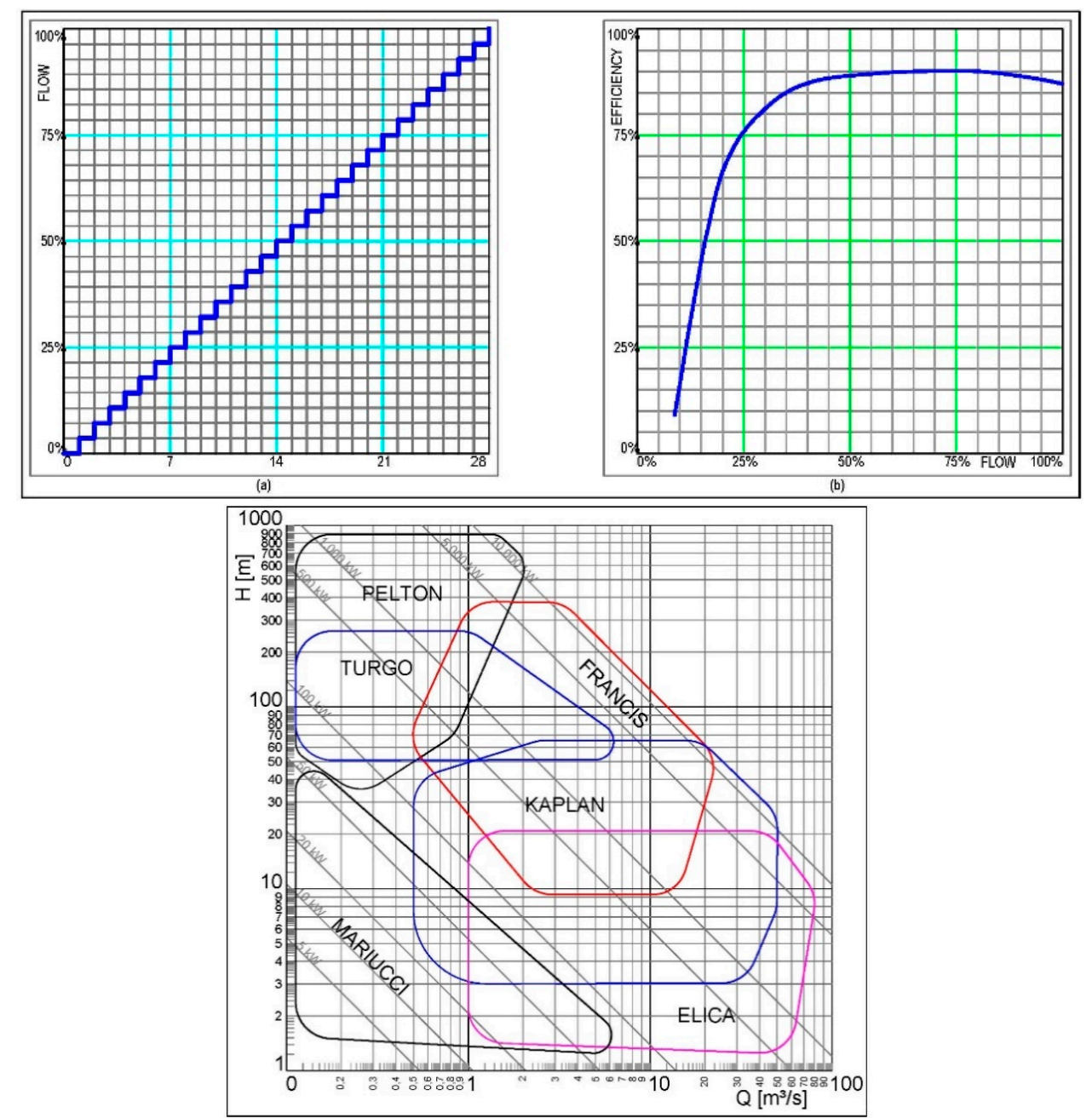

(c)

Figure 54. (a) Progressive flow according to the opening of the valves. (b) Efficiency as a function of flow; (c) range of flow rates and heads. 
The experimentally measured efficiency is represented in Figure 54b: ranges from $85 \%$ to $90 \%$ from $35 \%$ of the design flow rate to the design flow rate, which is the maximum one. These data were measured in 2014 on the real power plant in Rasiglia (Italy), with installed power of 5 kW. In Figure 54c, the operating hydraulic range of the turbine is compared with traditional turbines.

The machine components are built with the following materials:

- distributor, nozzles, impeller, valves, rods for opening and closing the valves, sliding bands, templates for openings in the floors, are built with $\mathrm{X} 2 \mathrm{CrNi} 1810$ steel.

- load-bearing structure is built with galvanized steel.

- transmission shaft, lower and upper supports, are built with S235 JO steel.

The unit cost of the turbine for a theoretical power input of $118 \mathrm{~kW}$, corresponding to a flow rate of $4.00 \mathrm{~m}^{3} / \mathrm{s}$ and a head $H=3.00 \mathrm{~m}$, that will be soon installed with a turbine runner of $2.20 \mathrm{~m}$ in diameter and rotating at $50 \mathrm{rpm}$, is $€ 1300,00 / \mathrm{kW}$, lower than low head Kaplan turbines [70,71]. The unit includes: the turbine, the speed multiplier, the electric generator, the hydraulic system, the electrical panels and the software and hardware, excluding building works. The entire machine is assembled in the workshop, then by a crane it is lifted and inserted into the circular openings of the floors and bolted to the attic of the technical room.

A future application of this system could be the turbine installation in a pressurized pipe, where the final section of the pipeline is a classic spiral scroll (Figure 55).

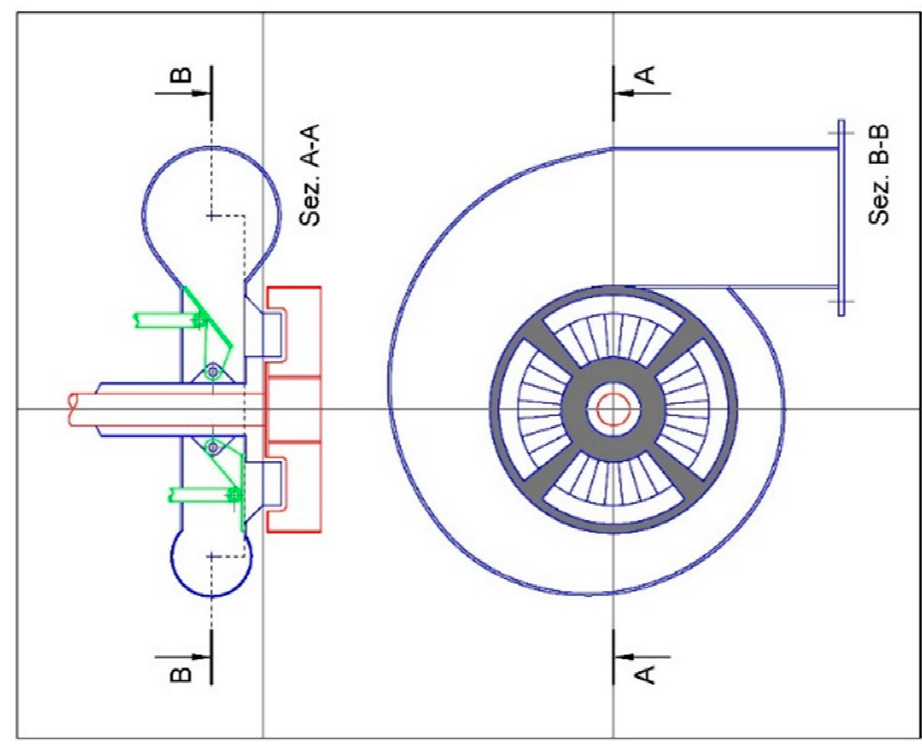

Figure 55. The turbine in a forced conduit system.

\section{Rehabilitation of a Low-Head Gravitational Vortex Site with an Improved Vortex Turbine}

In many countries all over the world, a significant part of hydropower has not been developed due to the head limitations and design constraints of traditional turbines. The complexity with these low head sites is the large flow required to reach sizable power production. When trying to utilize classic turbine designs for these sites, debris, cavitation, and an uneconomical scaling of the rotor hinder a successful business case. Therefore, new turbines are emerging on the market, like the vortex turbine and water wheels $[10,71]$. These machines allow the exploitation of low head sites at low cost and using civil works constructed with local labor when using a new non-conventional turbine. Among new low head hydropower converters, the novel vortex turbine [10] has been developed and installed in several installations all over the world. Previous Gravitational vortex turbine designs have shown the usefulness of the technology, but the rotor and basin dimensions were such that the solution reached a higher cost per $\mathrm{kW}$. The current vortex turbine design, as proposed by Turbulent, 
was designed to solve these issues and make the vortex technology a realistic option for hydropower development of these low head sites in small and mid-sized rivers and increase the use of vortex turbines for rural electrification. Depending on model size, the Turbulent vortex turbine can operate within a head range of $1-4.5 \mathrm{~m}$ and a flow range of $0.7-9 \mathrm{~m}^{3} / \mathrm{s}$.

The current case study describes the replacement of the rotor, drivetrain, and power electronics of a previously installed gravitational vortex turbine in Bali. This turbine is installed to provide sustainable energy to the Green School by using the small head difference and large flow of the Ayung River. The site has a head of $1.85 \mathrm{~m}$ and a flow of $1.5 \mathrm{~m}^{3} / \mathrm{s}$. The previous turbine was not able to produce more than $5 \mathrm{~kW}$ in practice, possibly due to technical problems with the generator. The rotor also required a large amount of maintenance as it would get blocked by debris (coconuts, branches, palm fronts and stones) which are abundant in the Ayung river. After a flood destroyed the drivetrain and power electronics a new solution was needed.

\subsection{Design Parameters}

Based on the site head and flow, a turbine diameter of $1.18 \mathrm{~m}$ was calculated, which corresponds to a required basin diameter of $3.9 \mathrm{~m}$. The outlet is usually designed to be free (discharging to atmospheric pressure) or can be equipped with a diffuser (negative pressure at the outlet). However, for this site, the existing basin has a diameter of $7.9 \mathrm{~m}$. The outlet is submerged by about $15-20 \mathrm{~cm}$ (Figure 56c), that would lead to Carnot shock losses [72], and the construction of a diffuser was not feasible due to site constraints.

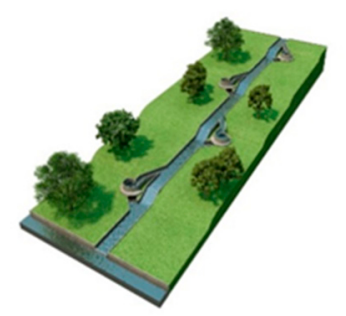

(a)

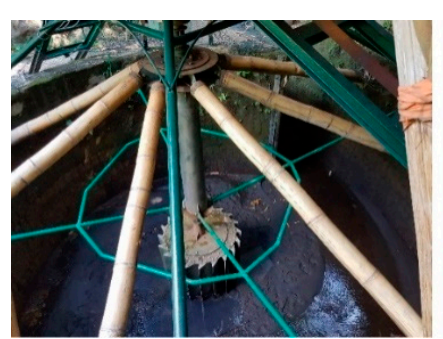

(b)

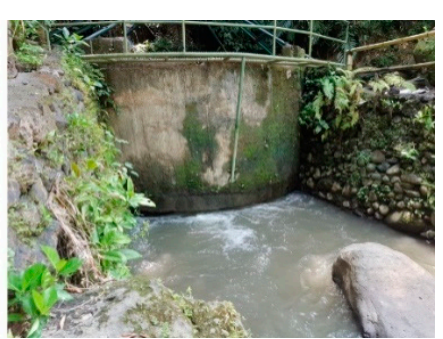

(c)

Figure 56. (a) Turbulent vortex turbine concept; (b) previous vortex turbine and (c) submerged outlet at nominal flow.

These factors necessitated a customized solution for the retrofit so that the performance measures are acceptable even for the oversized basin. A series of free-surface CFD simulations were conducted using the interFoam solver in OpenFOAM (Figure 57) to size the rotor and validate the performance of the retrofit. After commissioning, it was found that the measured performance of the plant slightly exceeds performance predicted by CFD, possibly due to some uncertainty in outlet tailwater level measurements. The turbine size and performance measures are listed in Table 11.

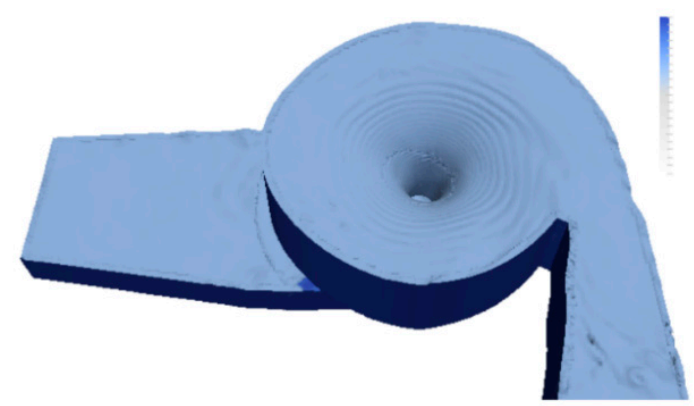

Figure 57. Computational Fluid Dynamics (CFD) model for Bali turbine. 
Table 11. Turbine size and performance metrics.

\begin{tabular}{cc}
\hline Parameter & Value \\
\hline Impeller diameter & $1.18 \mathrm{~m}$ \\
Blade row height & $0.44 \mathrm{~m}$ \\
Speed & $96 \mathrm{rpm}$ \\
Nominal flow & $1.5 \mathrm{~m}^{3} / \mathrm{s}$ \\
Predicted head & $2.05 \mathrm{~m}$ \\
Measured & $1.85 \mathrm{~m}$ \\
Net predicted power & $14.1 \mathrm{~kW}$ \\
Net Measured power & $13 \mathrm{~kW}$ \\
Predicted hydr. efficiency & $54.6 \%$ \\
Measured hydr. efficiency & $55.8 \%$ \\
\hline
\end{tabular}

This optimization allows the Turbulent design to produce $13 \mathrm{~kW}$ with the current site. Due to the design of the Turbulent turbine, this could be achieved with a relatively small and lightweight turbine (rotor and drive train are approximately 6 times smaller than the previous gravitational vortex turbine and weight is limited to $550 \mathrm{~kg})$ and at a relatively low cost $(0.07 \mathrm{EUR} / \mathrm{kWh}$ incl. maintenance).

The turbine itself was installed in less than one day (Figure 58), commissioning was completed after three days. The flood-proof design was tested just one day after installation during an evening storm (Figure 59). The turbine not only survived the flood but also let all the debris and bed load pass through without harm to the blades or structure.
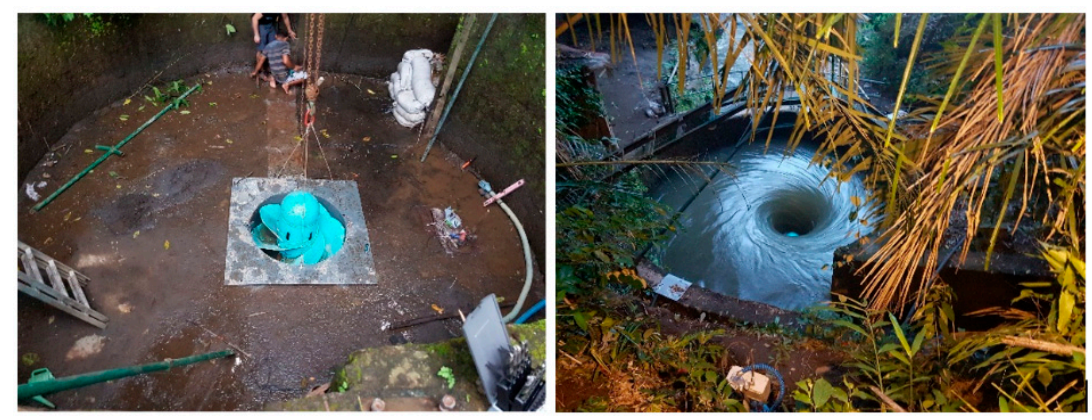

Figure 58. Installation with winch (left) and manual lifting (right) first test run.
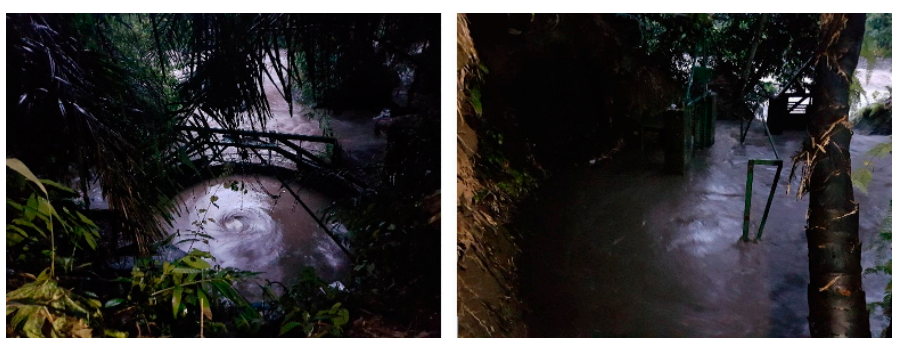

Figure 59. First flood event with inlet completely submerged.

\subsection{Low Ecological Impact}

The design of a vortex turbine allows it to be installed in rivers with minimal head differences. In the case of this site in Bali, the head was already available due to a natural cascade of boulders. To ensure enough water would deviate into the turbine, a small gabion weir ( $2.5 \mathrm{~m}$ width, $1.5 \mathrm{~m}$ height) was built (Figure 60, left side). A 20-meter bypass canal brings the water to the turbine after which it is returned straight back into the river. This way the river flow is not interrupted and no barrier for fish movement is created. 


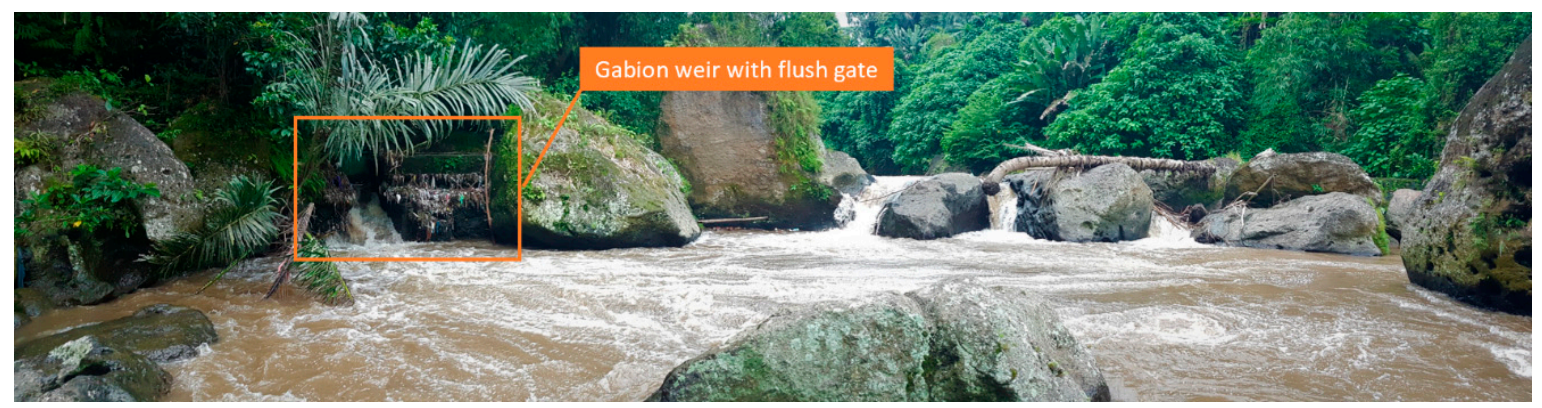

Figure 60. Intake structure.

\subsubsection{Ecologically Improved Design}

Fish friendly turbines are becoming more and more important, especially under the EU water framework. With that in mind, the turbine was designed according to criteria from previous research on turbine fish-friendliness by Amaral et al. [73], Cada et al. [74] and Cook et al. [75]. Potential damage mechanisms are identified in four categories: mechanical injury (strike, grinding, etc.), pressure (exposure to decreased pressure and rate of pressure decrease), shear/turbulence and cavitation [74]. Out of these, mechanical injury mechanisms are considered dominant, and among these, a leading-edge blade strike is assumed likely to be the primary cause of fish mortality due to turbine passage in many hydropower plants [73]. Physoclistous fishes are very susceptible to injury/mortality due to sudden pressure decrease that leads to swim bladder rupture or internal hemorrhaging [76]. In this study, the considered turbine is evaluated against thresholds for leading-edge strike, barotrauma and shear flow-related injury, using steady-state MRF (multiple-rotating frame) CFD simulations.

\subsubsection{Leading Edge Strike}

Amaral et al. [73] conducted two-species experiments with different fish lengths $(L)$ and blade thicknesses $(t)$, and found that there was little or no fish mortality (97-100\% total survival rate) for blade strike speeds (relative velocity between fish and blade) up to $5 \mathrm{~m} / \mathrm{s}$. Figure 61 a reproduced from that study [73] shows the data points and linear regression relations between total survival percentage and strike speed for varying $L / t$ ratios. In Figure $61 \mathrm{~b}$, the relative velocities for the studied vortex turbine are plotted. The relative velocities are almost entirely below the $5 \mathrm{~m} / \mathrm{s}$ threshold throughout the flow passage area, except very tiny portions at boundary layers shown in grey. Following the reasoning by Cook et al. [75], such tiny non-compliant volumes minimize damages to fish.

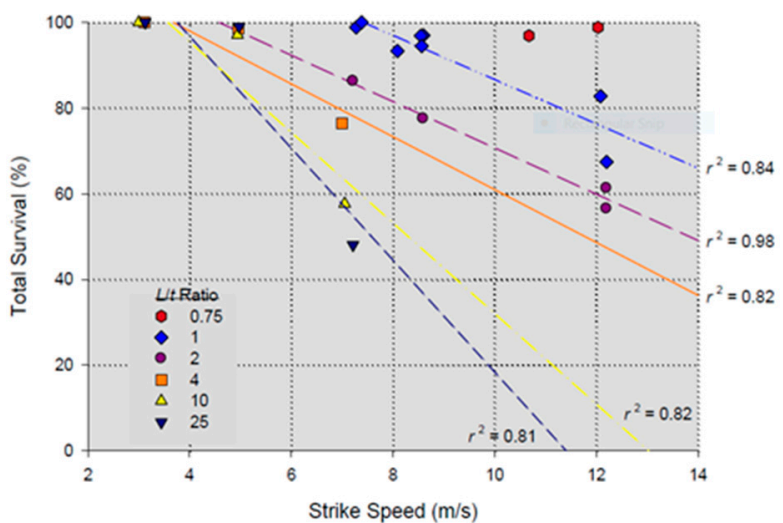

(a)

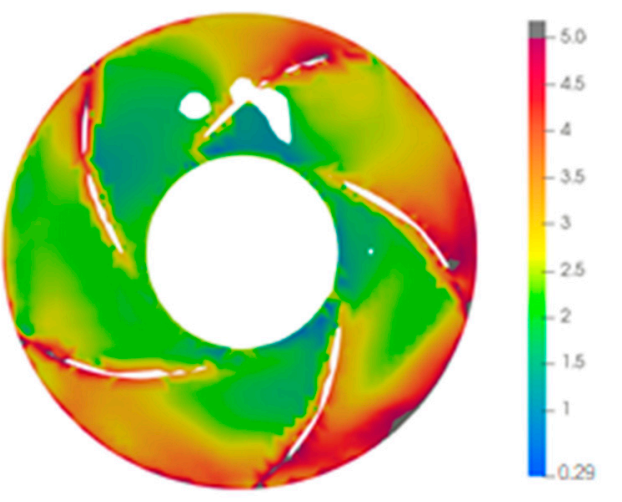

(b)

Figure 61. (a) Total survival vs. strike speed for different $L / t$ ratios; (b) relative velocity $(\mathrm{m} / \mathrm{s})$ at leading edge ((a) reproduced with permission from authors). 


\subsubsection{Pressure Decrease}

Based on a review of past studies, Cada et al. [74] found that when the pressure drop is less than $40 \%$ of the fish acclimation pressure (i.e., the pressure ratio $>60 \%$ ), then there is little or no fish-mortality. Between 1973-1986, the water level was measured to be 0.55-0.88 m in the Ayung river [77]. The maximum acclimation depth of fishes in the Ayung river was conservatively assumed to be twice the maximum depth $(1.76 \mathrm{~m})$ in the absence of more specific data. The equivalent maximum acclimation pressure, $P_{\mathrm{a}}=17.19$ psa. From Figure 62, the minimum exposure pressure, $P_{\mathrm{e}}=-1.1 \times 10^{4}$ $P_{\mathrm{a}}($ relative $)=13.1$ psa. Thus, the ratio $P_{\mathrm{e}} / P_{\mathrm{a}}=76 \%$, which is safely above the threshold limit $(60 \%)$.
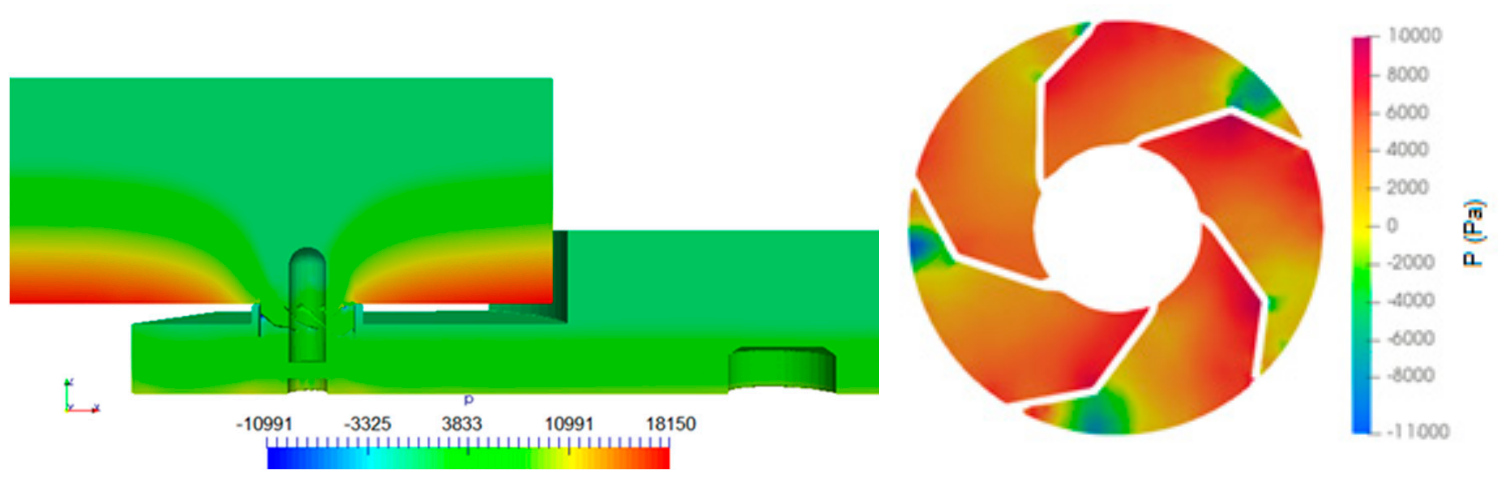

Figure 62. Pressure plot shown on a longitudinal section, and Pressure plotted on cross-section.

\subsubsection{Rate of Pressure Decrease}

Based on the experimental results by Abernethy et al. [76], Cook et al. established $-500 \mathrm{psi} / \mathrm{sec}$ as the fish-friendly threshold of pressure decrease rate for Alden turbine design criterion [75]. In this study, we follow the same criterion and compare the actual pressure drop rate with this value. While there is some uncertainty regarding fish behavior during runner passage, previous studies have assumed fishes to follow flow streamlines like mass-less particles (e.g., Richmond et al. [78]). Under this assumption, the rate of pressure decrease (as experienced by a fish as it travels through the turbine) can be calculated using the material derivative of pressure that contains an advection part and an unsteady part:

$$
\frac{D p}{D t}=\frac{\partial p}{\partial t}(\text { unsteady })+\vec{V} \cdot \nabla(p)=0+\vec{V}_{r} \cdot \nabla_{r}(p)=\vec{V}_{r} \cdot \mathrm{R}(\theta) \nabla(p)
$$

where $\frac{D p}{D t}$ is the material derivative of pressure, $\frac{\partial p}{\partial t}$ is the transient (unsteady) part, $\vec{V}, \nabla(p)$ and $\vec{V}_{r}, \nabla_{r}(p)$ are fluid velocities and pressure gradients in stationary and rotating coordinate frames respectively, $R(\theta)$ is the rotation matrix with $\theta$ being the angle between the two frames. In a steady state MRF CFD simulation, the rotating coordinate frame instantaneously coincides with the CFD coordinate system, $\nabla(\mathrm{p})=\nabla_{r}(p)$. Thus, $\frac{D p}{D t}=\vec{V}_{r} \cdot \nabla(p)$. The RHS is evaluated in CFD and plotted in Figure 63a (longitudinal section) and Figure 63b (cross-section at mid-height of impeller). Throughout the flow area, except at localized hotspots, the pressure drop rates are very gradual. The maximum pressure drop rate is less than $116 \mathrm{psi} / \mathrm{s}$ and safely lower than the threshold limit of $500 \mathrm{psi} / \mathrm{s}$.

\subsubsection{Shear Strain Rate}

Based on previous studies, the fish-friendly threshold for the shear strain rate was taken as $500 \mathrm{~s}^{1}$ (Cook et al. [75]). The fluid strain rates in XY, YZ and ZX planes are plotted in Figure 64a-c, respectively. The strain rates are safety limited below the safety threshold except very tiny areas shown in grey, which can be deemed inconsequential (Cook et al. [75]). 


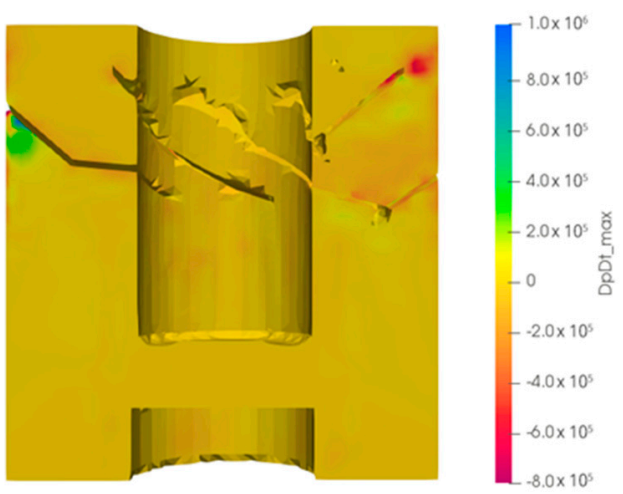

(a)

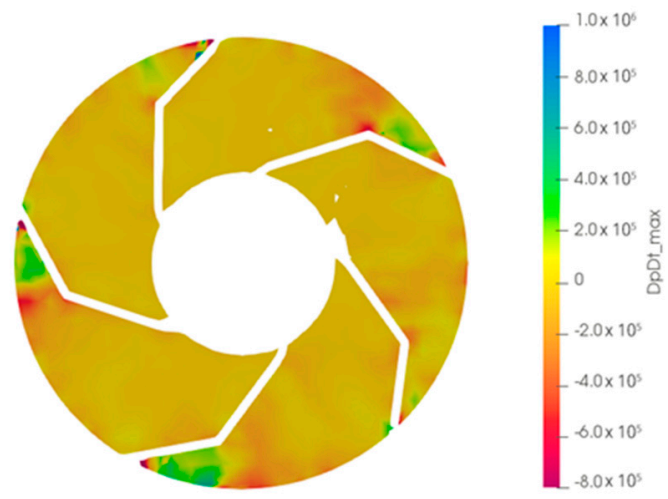

(b)

Figure 63. Dp/Dt over ZX plane (vertical section, on the left (a)), Dp/Dt over XY plane (cross-section, right $(\mathbf{b}))$.

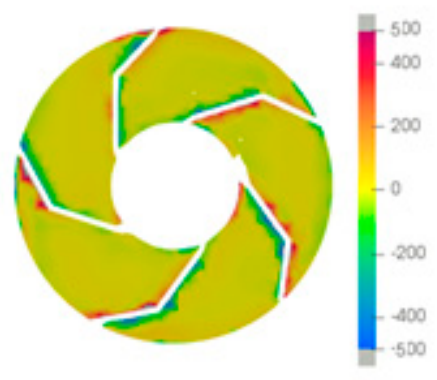

(a) $\mathrm{XY}$ strain rate $\left(\mathrm{s}^{-1}\right)$

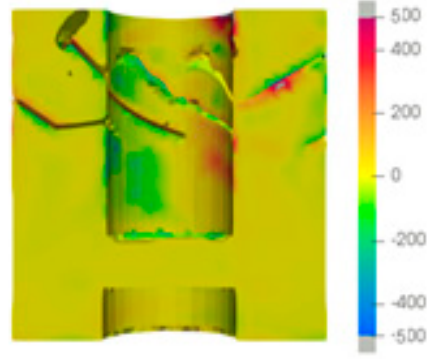

(b) $\mathrm{YZ}$ strain rate $\left(\mathrm{s}^{-1}\right)$

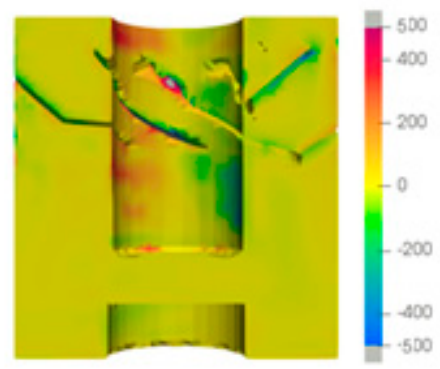

(c) ZX strain rate $\left(\mathrm{s}^{-1}\right)$

Figure 64. Strain rates along different planes, (a) XY, (b) YZ, (c) ZX.

\subsection{Conclusions}

The novel Turbulent vortex turbine design, combined with the refurbishment of the existing gravitational vortex basin structure, can be seen as a successful case that demonstrates that a vortex turbine can produce energy at an acceptable cost while also minimizing ecological impact. The current study indicates the fish-friendliness of the turbine based on CFD results and established fish-friendliness metrics. However, detailed on-site testing will be conducted in the near future to better investigate this aspect, which is a complex problem depending also on fish behavior.

\section{Very Low Head Turbines on Navigation Locks}

Despite the need for green energy to reduce the climate impact of the power sector, in the last few years, hydropower projects are struggling to obtain the permits to construct even for medium and mini-power plants. Therefore, developers aim to find solutions that use existing sites and have a very low environmental impact. Navigation locks present a perfect example of existing facilities with hydro turbines, instead of the gates, that regulate the flow, producing energy otherwise wasted. The Very Low Head machine is a promising technology in this context [79]. Two different sites were installed following the previously described philosophy. The first one is located in Canda (Rovigo, Italy) on the Canal Bianco, and it has been commissioned at the end of 2016. The main features of Canda plant are canal height $7 \mathrm{~m}$, canal width $9.5 \mathrm{~m}$, gross head $3 \mathrm{~m}$ and usable annual mean flow $16.5 \mathrm{~m}^{3} / \mathrm{s}$. Figure 65 shows the existing civil structure of the navigation lock. 


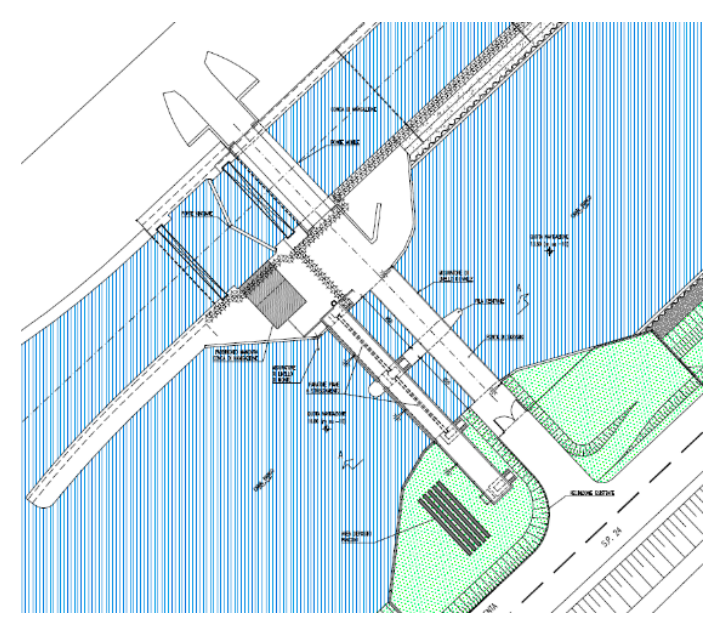

(a)

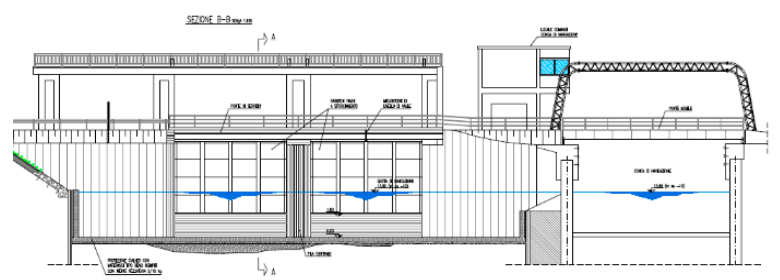

(b)

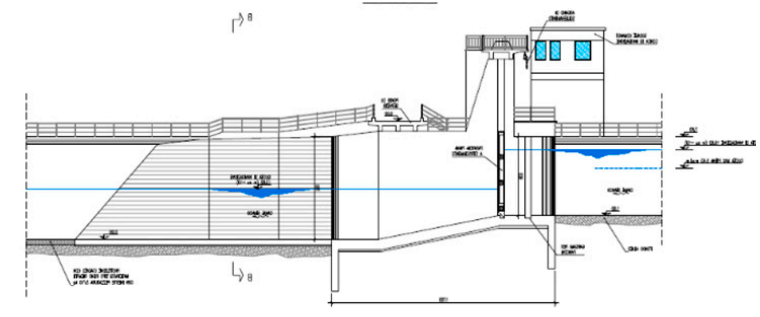

(c)

Figure 65. (a) top view of the existing civil structure; (b) cross-section of (a); (c) longitudinal section of (a).

The second one is located in Bussari (Rovigo, Italy) on the Canal Bianco, and it was commissioned in the first half of 2017. The main features of the Bussari plant are canal height $6.3 \mathrm{~m}$, canal width $8 \mathrm{~m}$, gross head $2.56 \mathrm{~m}$ and usable annual mean flow $25 \mathrm{~m}^{3} / \mathrm{s}$. Figure 66 shows the existing civil structure of the navigation lock.

As can be seen in Figures 65 and 66, the sites are characterized by low head and during the authorization procedures, no additional civil works were allowed. A technology that fulfills the aforementioned requirements is the VLH (Very Low Head) turbine. In the VLH hydro turbines the generator and the distributor form a simple unique self-supporting structure (Figure 67), placed obliquely in the derivation canal. The bulb contains the Kaplan runner and the Permanent Magnet Synchronous Generator (PMSG). In particular, the generator stator is placed in a central position in solidarity with the support of the impeller and the rotor magnets are housed inside the Kaplan runner. The generating unit is fully submersible, allowing a silent operation and a low-visual impact [80-82]. Table 12 represents the main data of Canda e Bussari machines. 


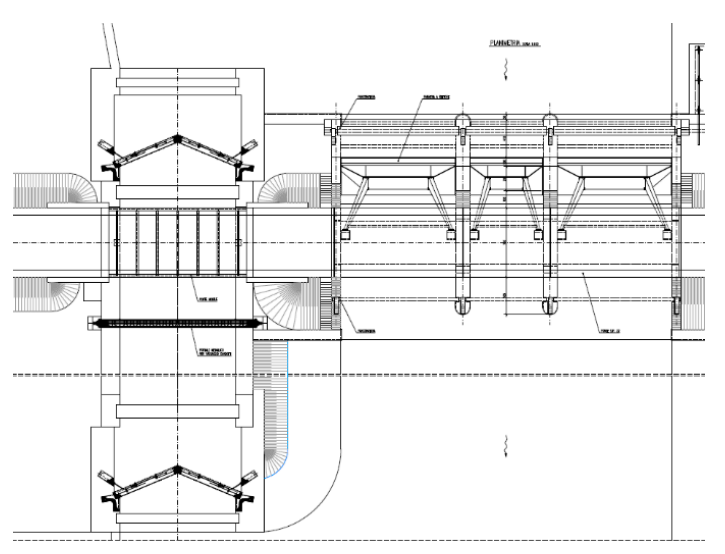

(a)

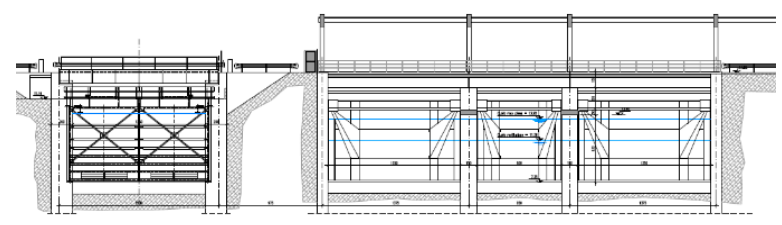

(b)

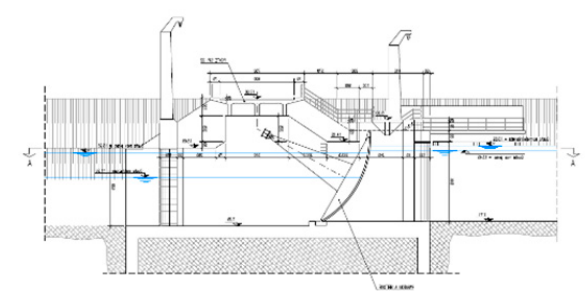

(c)

Figure 66. (a) Top view of the existing civil structure; (b) cross-section of (a); (c) longitudinal section of (a).

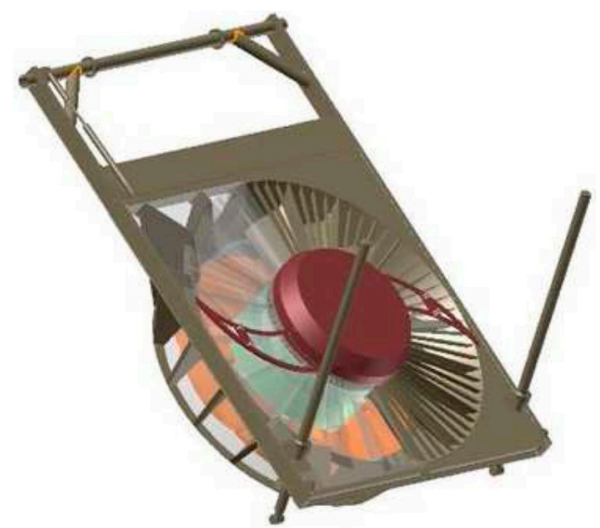

Figure 67. Front view of Very Low Head (VLH) turbine with its supporting structure. 
Table 12. Canda and Bussari machine data.

\begin{tabular}{cccc}
\hline Description & Units & $\begin{array}{c}\text { Value } \\
\text { (Canda) }\end{array}$ & Value (Bussari) \\
\hline Rated Power & $\mathrm{kW}$ & 256 & 481 \\
Min-Rated-Max speed & $\mathrm{rpm}$ & $34.9-55.2-58.6$ & $20.3-32.1-34.1$ \\
Unit Speed $\mathrm{N}_{11}$ & - & 100 & 100 \\
Unit Discharge $\mathrm{Q}_{11}$ & - & 0.68 & 0.625 \\
\hline
\end{tabular}

\subsection{Steel Structures}

Due to the different canals' width, two sizes of VLH turbines were chosen. In Canda power plant two (2) VLH 3150 (runner diameter of $3150 \mathrm{~mm}$ ) were installed with a total power of $2 \times 256 \mathrm{~kW}$ achieving an annual production capacity of $2888 \mathrm{MWh}$, whereas in Bussari one (1) VLH 5000 (runner diameter of $5000 \mathrm{~mm}$ ) was installed with a total power of $1 \times 481 \mathrm{~kW}$ achieving an annual production of $2751 \mathrm{MWh}$.

Figure 68 shows the steel structures for both the plants, where it can be noted the possibility to lift the turbines, with two hydraulic cylinders, above the water level for maintenance purposes and during the floods.

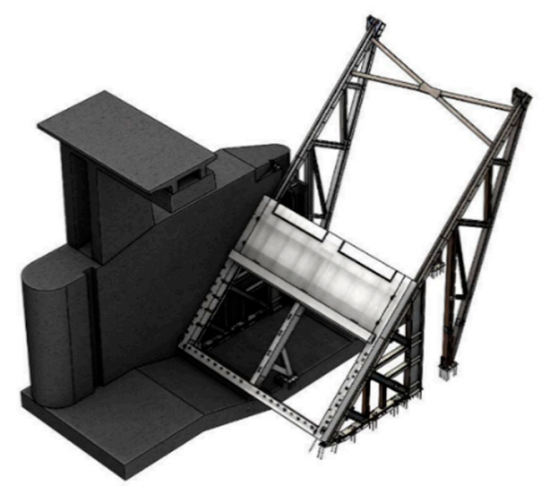

(a)

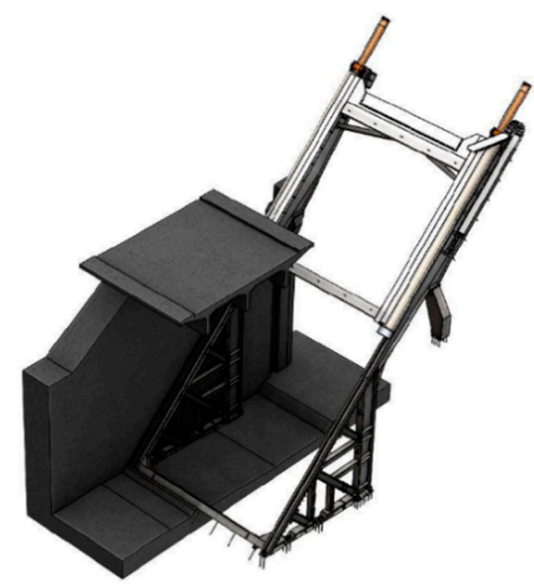

(c)

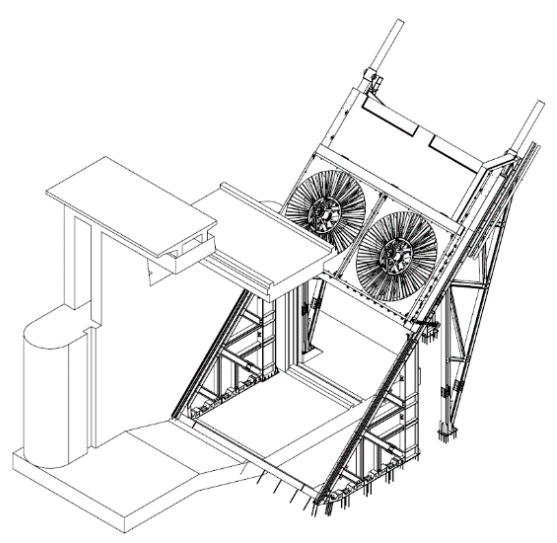

(b)

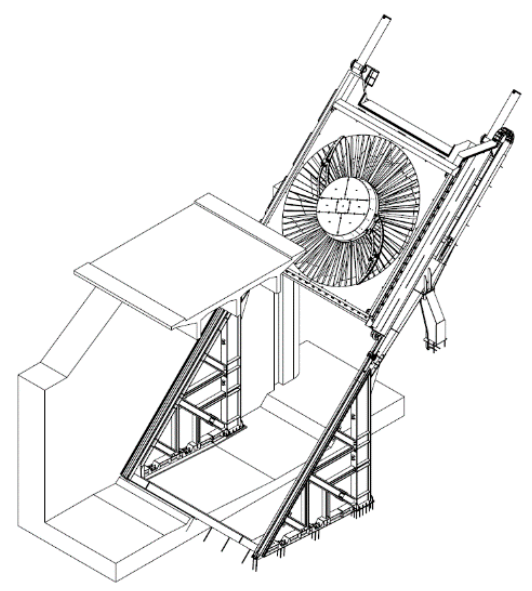

(d)

Figure 68. (a) Canda steel structure in the existing civil works with the mobile part in operational position; (b) Canda steel structure plus turbines with the mobile part in maintenance position; (c) Bussari steel structure in the existing civil works with the mobile part in maintenance position; (d) Canda steel structure plus turbines in the existing civil works with the mobile part in the maintenance position. 
A FEM analysis was carried out to verify the mechanical stresses on the structures; as it can be noted in Figure 68a Canda steel structure requires additional iron beams to sustain the turbines in out of water position. Furthermore, another critical point was to verify the mechanical stresses of the mobile part of Canda project in the worst-case scenario (maximum level of the upstream and a dry downstream). Both the steel structures were directly anchored on the existing civil works (Figure 69).

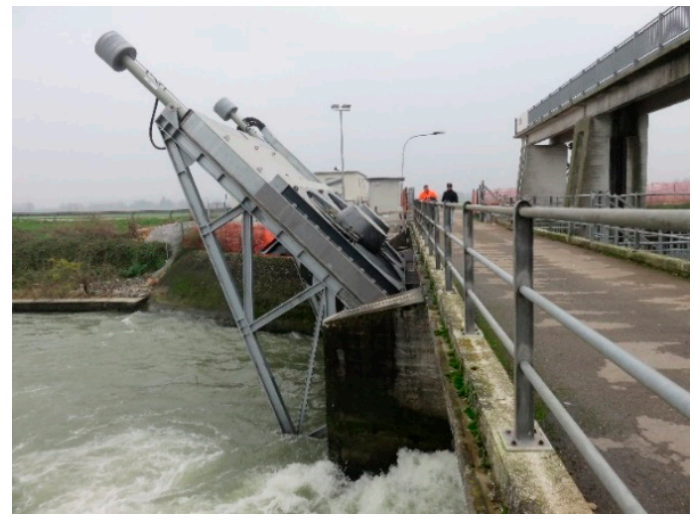

(a)

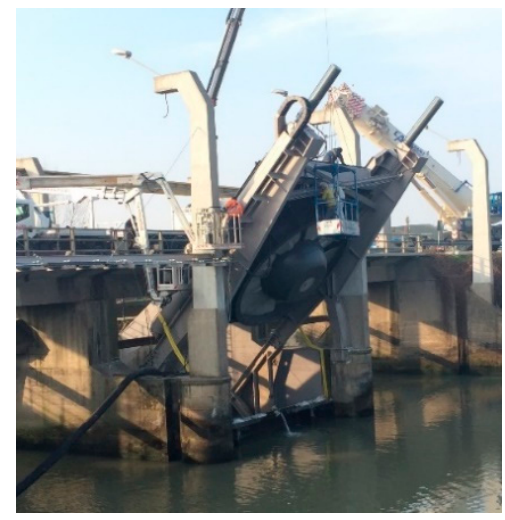

(b)

Figure 69. (a) Canda turbines during a flood; (b) Bussari turbine during maintenance activities.

\subsection{Costs}

Using the existing civil works has led to a minor cost per $\mathrm{kW}$ installed. Comparing similar projects with the same VLH turbines the required civil costs were reduced by $80 \%$; the Canda and Bussari civil works are related to the control building and cable trenches; the design of a dedicated steel structure instead of a standard one produced an increase of $40 \%$ for the cost of mechanical structures. The total cost of Canda and Bussari projects was 3950 and $3650 € / \mathrm{kW}$ respectively, where the similar plant in which civil works were needed had a total cost of $5000 € / \mathrm{kW}$, so it is possible to assume that exploiting the existing civil works can lead to a cost reduction between 20 and $30 \%$.

\subsection{Control}

Both the power plants are dispatch with a water level control, to guarantee the correct upstream and downstream levels using the available flow. The control algorithm adjusts the pitch of the blades and then an innovative Maximum Point Power Tracker (MPPT) control (Figure 70), described in [83], adjusts the turbine speed exploiting the VLH variable speed feature granting a very wide flat efficiency curve and a maximum power operation point.

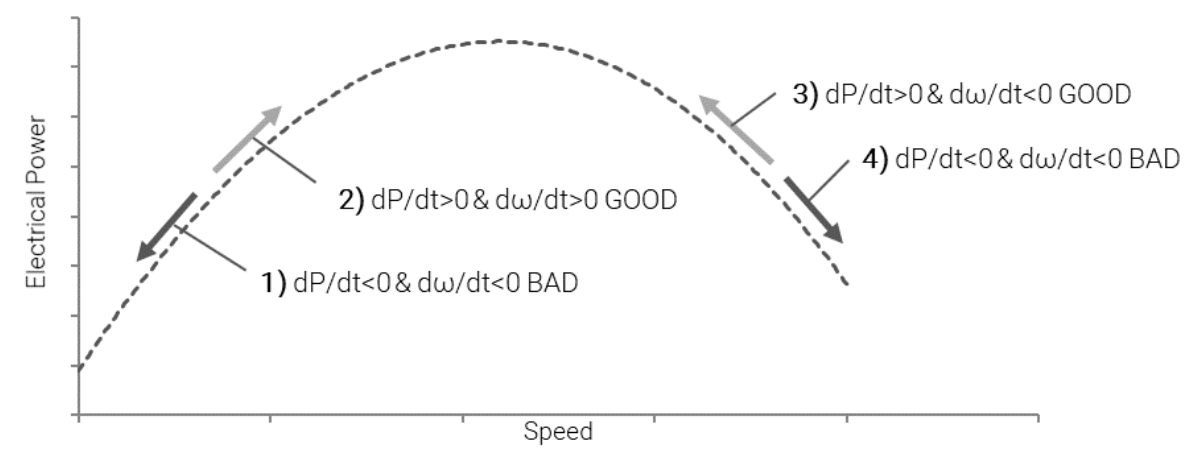

Figure 70. MPPT control principles.

For the given water head and blades pitch angle, the generation chain (turbine-generator-frequency converter) presents a behavior as it can be seen in Figure 70. The basic concept of the proposed control 
strategy is to change the generator speed step-by-step, continuously monitoring the behavior of the system, until the maximum power point is reached.

Table 13 shows the selection criteria used for the speed variation. The regulator acts on the speed and evaluates the results (in terms of power delivered to the grid) of its action. Four operating conditions can be distinguished.

Table 13. Bussari machine data.

\begin{tabular}{cccc}
\hline $\mathrm{dP} / \mathrm{dt}$ & $\mathrm{d} \boldsymbol{\omega} / \mathbf{d t}$ & Direction & Action \\
\hline$<0$ & $<0$ & Bad & Increase speed \\
$>0$ & $>0$ & Good & Increase speed \\
$>0$ & $<0$ & Good & Decrease speed \\
$<0$ & $>0$ & Bad & Decrease speed \\
\hline
\end{tabular}

The blades, as is the case with the traditional regulator, are controlled to keep the upstream water level at a constant value. When a stable condition (e.g., no blades movement, no gates operation, etc.) is reached, the MPPT controller starts its action.

\subsection{Conclusions}

Two case studies of exploiting the available low head on navigation locks have been presented. A properly designed steel structure allows the installation of VLH turbines with no additional civil works and the control system of the power plant grants the correct management of water levels without the use of the existing gates but using the turbines as flow modulators.

\section{Discussion and Conclusions}

The case study collection shows engineering insights on new technologies and more sustainable methodologies to improve the hydropower sector, like the hydropower exploitation of existing structures to avoid interruption of new rivers and social diseases [84], turbines with better ecological behavior and new software and tools for a better governing and digital control of hydro schemes.

New speed governors can be used to optimize efficiency and to reduce trip events. The case study here examined reduced the turbine overspeed from $129 \%$ to $123 \%$, with a distributor closing law based on two-speed gradients in combination with the slow closing of the runner blade. This solution has the benefit to reduce the overspeed and, in the specific case, to avoid the uplift of the runner, thus improving the life span and reducing maintenance. New control systems, by means of digitalization, can also allow us to understand at any time the health state and behavior of all essential components of the system and to estimate quantities throughout the whole system. By replicating the hydraulic transients of the power plant, it is possible to know the pressure variations throughout the waterways, assessing the penstock fatigue. The case study of La Bâtiaz high head Pelton turbine power plant, in Switzerland, showed the significant contribution of ancillary services to penstock fatigue, particularly at the top of the penstock. The Digital Twin of the hydropower plant paves the way for turbine control parameter optimization to reduce penstock fatigue and thus increase service lifetime. The digitalization was also implemented by several companies, increasing in one case the production of the plant by $1.2 \%$ on an annual basis in the discussed case study, by subdividing the load on various generation units. Better digitalized controls were also applied to a Kaplan installation: the optimized turbine conjugation curve translates into increased machine efficiency between $0.5 \%$ and $0.8 \%$, depending on the operating condition of the unit. The maximum benefit was achieved in high loads. The additional earning was $585 \mathrm{MWh}$ in the last 2 years, with respect to $117 \mathrm{GWh}$ in 2 years. The sediment management solution adopted in the Pontecosi plant, based on a fully automatic floating platform for the continuous removal of sediment, transfers the sediment through a transfer line to a point downstream of the reservoir to return to the river the sediments, minimizing impacts on sediments. 
New strategies to reduce environmental impact have also been addressed. For example, oil-free Kaplan turbine and new bearing systems, like a polycrystalline diamond (PCD) bearing-shaft assembly for the hydrokinetic RivGen Power System in the Kvichak River. The latter allows us to overcome the challenges of sediment-filled water and eliminate the need to surface the system to inspect and maintain the bearing-shaft assembly, removing the need for contaminating lubricants and ongoing maintenance. However, this is not the only oil-free solution existing on the market: the low friction and good insulating properties of PTFE allows us to reduce the requirement for "oil-jacking" (where high-pressure oil is injected into the gap between the bearing and counterface during start-up to separate the surfaces until a self-sustaining film can be formed) as well as offering a performance improvement. The Sant Antonio hydroelectric plant uses an efficient solution to reduce its environmental impact. The construction of two large underground demodulation reservoirs has led to significant environmental improvements by reducing the effects of hydropeaking downstream of the plant. The analysis of the changes in the available habitat area for fish showed how the presence of the underground demodulation reservoir guarantees a general increase in habitat availability.

New reaction fish-friendly turbines are also under development, with survival rates of more than $98 \%$. The vortex turbine is a low head turbine with $55 \%$ efficiency, $0.07 € / \mathrm{kWh}$ and good ecological behavior: indeed, the maximum pressure drop rate is less than $116 \mathrm{psi} / \mathrm{sec}$ and safely lower than the threshold limit of $500 \mathrm{psi} / \mathrm{s}$, with the relative velocities that are almost entirely below the $5 \mathrm{~m} / \mathrm{s}$ threshold throughout the flow passage area. Nevertheless, the fish-friendly behavior of a turbine is a complex matter, depending also on fish behavior, and that cannot be solved only by CFD results. Therefore, field tests are necessary and will be conducted in the future, as already done for water wheels and Archimedes screw turbines [85].

Hydropower can also be a driver for the local economy and the local sustainable development. In Maira Valley, the economic income from energy sales from hydropower was adequate to compensate the basic costs of the plants and the company, including the financial charges for the repayment of the debt and interest, and to cover externality plans, strategic structures for the development of the local socio-economy, the setting of new projects, as well as stakeholders remuneration. Territorial development for example created non-precarious work and stimulated social positivity through various initiatives, like a mountain refuge and some economic activities. This example was successful, especially because there was no need for population re-allocation, which would have caused social problems, as it often happens with big dams [83,85].

One other discussed topic is the energy recovery by hydropower installed existing hydraulic structures. In San Michele dei Mucchietti locality, on the Secchia River, in the Municipality of Sassuolo (MO) and Castellarano (RE), an important weir was built in the 1980s in order to provide water for irrigation to Modena and Reggio Emilia plane through two historic channels, no longer usable due to the lowering of the river bed, and now used to serve a hydropower plant, for a total of 1800 $\mathrm{kW}$. The use of existing structures has allowed a substantial and quantifiable saving of 300,000 $€$, avoiding a complex cutting operation of the bank of the lateral reservoir and the restoration of its waterproof structure. Costs were avoided for the construction of protection screens, huge stone boulders, walls higher than the two-hundred-year period flood level. A reduction in the costs of the work of $200,000 €$ was estimated. Therefore, the total saving was $277 € / \mathrm{kW}$, that compared to an average installation cost of $1300-8000 \mathrm{USD} \$ / \mathrm{kW}$ [86] is a cost-saving of 3.4-25\%. An example of hydropower development on existing dams was also described, which uses the ecological flow: through a Pelton turbine, a global medium efficiency of $92.7 \%$ was achieved. The cost spent on the execution of the plant was approximately $2,086,000 €$ while the overall cost consisted of 3,000,000 $€$. Therefore $8150 € / \mathrm{kW}$ was the price for $1 \mathrm{~kW}$ of nominal power installed, which has led to a period of six years necessary to return the investment. If the current plant was not adopted it would have implied the use of two turbines, leading to an overall cost increase of $60 \%$, management and maintenance charges almost doubled, with the same total efficiency. The time required to return the investment would also have been increased to nine years. 
Energy recovery in drinking water networks is also a sector in rapid development. The installation of a hydro plant in Susa Valley was discussed, for a total power of $2380 \mathrm{~kW}$. The overall average production expected from the ordinary operation will be in the order of $12 \div 13 \mathrm{GWh} /$ year. An irrigation plant was converted to a multi-purpose plant, through the installation of a counter pressure Pelton turbine, where previously a pressure reducing valve dissipated the present hydrostatic energy. Several innovative elements were introduced in this plant. The plant certainly has to be seen as a benchmark example for multi-purpose use of water, as it uses the processed water both for hydropower generation and irrigation. Therefore ecological consequences on the exploited stream are absent as the water is already used for irrigation. A counter-pressure turbine is a good technology to be installed in already existing systems. Not needing usually a new powerhouse, it allows to reduce civil works to a minimum and therefore to optimize costs of investment. An interesting case study is also the variable speed turbine of $150 \mathrm{~kW}$ in an aqueduct. The turbine was synchronized with bypass through a smart system to prevent excessive opening during transients that occurs at discharges lower than the maximum one: this feature avoids the valve to be opened more than required from users' demand and preserves pipe from buckling.

The last discussed topic is the low head hydropower. The Hydrostatic Pressure Machine (HPM) operates on sites with ultra-low hydraulic heads between 0.5 and $2 \mathrm{~m}$. At the optimal operating point in flow rate, or equivalently in rotation speed, the HPM efficiency is slightly less than $60 \%$, at $N^{*}=0.33$, which can be used as design value. The optimal angle of the shroud was also identified for a better design. Compared to classical water wheels, the HPM rotates faster, it is more compact and can exploit straight streams, but its efficiency is lower and has to be improved. The ecological impact on fish is not well known, and the high torque oscillations require a good design of the power take-off. The Mariucci turbine is a new action turbine, patented in 2017, with flow rates ranging from 0.3 to $6 \mathrm{~m}^{3} / \mathrm{s}$, head differences within $1.5-8 \mathrm{~m}$, and efficiencies of $90 \%$. The unit cost for a theoretical power input of $118 \mathrm{~kW}$, corresponding to a flow rate of $4.00 \mathrm{~m}^{3} / \mathrm{s}$ and a head $H=3.00 \mathrm{~m}$, that will be soon built with a turbine runner of $2.20 \mathrm{~m}$ in diameter and rotating at $50 \mathrm{rpm}$, is $1300 € / \mathrm{kW}$, lower than low head Kaplan turbines. This turbine can be adapted to the downstream water level. Its drawback is that it needs a chamber with water with a hydrostatic head; it is essential to avoid collision with fish, especially due to the smaller space among the runner blades with respect to Kaplan turbines. Another low head turbine of recent innovation is the vortex turbine, which was designed as a realistic option for hydropower development of low head sites in small and mid-sized rivers. Depending on model size, the described vortex turbine can operate within a head range of $1 \mathrm{~m}-4.5 \mathrm{~m}$ and a flow range of $0.7-9 \mathrm{~m}^{3} / \mathrm{s}$. The efficiency is around $55 \%$, which can be improved by acting on the blade shape and diffuser. Its fish-friendly behavior should be proved by tests, although CFD results indicate good ecological performance. Compared to water wheels and Archimedes screws, the Vortex turbine can exploit higher discharges employing a smaller runner, but it requires a big circular chamber and outlet/diffuser structures, and the efficiency is slightly lower. Future works should aim at better showing the comparison with existing low head (and free surface) technologies. In order to deal with higher flow rates, not exploitable by previous machines, the Very Low Head machine is a promising technology for low head hydropower applications. The VLH turbine was implemented in the Canda plant with a gross head of $3 \mathrm{~m}$ and a usable annual mean flow of $16.5 \mathrm{~m}^{3} / \mathrm{s}$, and in the Bussari plant, with a gross head of $2.56 \mathrm{~m}$ and a usable annual mean flow of $25 \mathrm{~m}^{3} / \mathrm{s}$. These plants use the existing civil works of navigation locks, which has led to a minor cost per $\mathrm{kW}$ installed. The total cost of Canda and Bussari projects was $3950 € / \mathrm{kW}$ and $3650 € / \mathrm{kW}$ respectively, while similar plants in which civil works were needed had a total cost of $5000 € / \mathrm{kW}$; hence it is possible to assume that exploiting the existing civil works can lead to a cost reduction up to 20 and $30 \%$, as confirmed in the study case of San Michele dei Mucchietti. New materials are being tested for the VLH turbine that can improve their efficiency [79]. Compared to Kaplan turbines, the VLH turbine is cheaper and with better ecological behavior, but it exhibits a lower efficiency and can only be applied to heads below $5 \mathrm{~m}$. 
These case studies confirm the fact that hydropower is a sector in development. New technologies and methodologies are being implemented to improve flexibility, efficiency and to reduce environmental impacts.

Author Contributions: All authors have read and agreed to the published version of the manuscript. The order of authors follows the case study order, except the first author, E.Q., who dealt with organization, writing of the paper (especially Introduction, References, Discussion and Conclusions), revision, contextualization, and case study collection. All the other Authors wrote their own case study, in particular:

- Novel speed governors for hydropower plants, by Compagnia Valdostana delle Acque, Italy.

- Digital twin for penstock fatigue monitoring, by Power Vision Engineering, HES-SO Valais, Electricite d'Emosson Switzerland.

- $\quad$ Better management by digitalization software and de-sedimentation techniques, Enel Green Power, Italy.

- An innovative solution to reduce the hydropeaking effect of hydroelectric power plants, by Eisackwerk, Bioprogramm S.c, Italy.

- $\quad$ Biological Validation of Improved Direct Turbine Survival at Ice Harbor Lock and Dam, by Voith Hydro, and USA Army Corp, USA.

- $\quad$ Diamond Bearings for hydrokinetic turbines, by US Synthetic, USA and ORPC Inc., USA.

- Hydropower as a driving force for Alpine territorial development, by Hydrodata and Intecno, Italy.

- Hydroelectric development of an existing multiple use reservoir, by Consorzio Emilia Centrale, Studio di Progettazione AISE Engineering, Italy.

- $\quad$ Mini hydro in environmental flow, by Ingegneri Consulenti, Italy.

- Hydropower from the Susa Valley drinking water network, by SMAT, Italy.

- Implementation of a multipurpose project on an irrigation plant by installation of an innovative counter pressure Pelton turbine, by Patscheider \& Partner Engineers Ltd., Italy.

- Variable speed application for Energy Recovery Hydropower in aqueducts, by ZECO Hydropower, Italy.

- Study of low-head hydrostatic pressure water wheels for harnessing hydropower of small streams, by Pytheas Tecgnology, France.

- $\quad$ The Mariucci turbine as evolution of the Girard turbine, by Teti srl, Italy.

- Rehabilitation of a low-head gravitational vortex site with an improved vortex turbine, by Turbulent, Belgium.

- $\quad$ The Very Low Head turbine in navigation locks, by STE Energy and RNB Hydro, Italy.

Funding: The research described in Section 6 was funded by the electric ratepayers of the Bonneville Power Administration (BPA) and the USACE Columbia River Fish Mitigation (CRFM) Program.

Acknowledgments: Thanks to Alberto Pistocchi for his general overview and comments. Section 3 was performed within the framework of the CTI project $\mathrm{n}^{\circ}$ 28112.1 PFIW-IW "Penstock Fatigue Monitoring". The authors would like to thank Innosuisse- the Swiss Innovation Agency-for its financial support in this work. The authors of Section 4 would like to thank everyone who contributed to developing these projects and communicating them: Sergio Adami; Cecilia Vicinanza; Gioacchino Bellia, Roberta Sardelli; Alessandro Benanti; Anna Di Cerbo; Michele Bologna; Elisa Maria Giannetto. Authors of Section 7 thank Jair Gonzalez, Tyler Kolste, and Franco Angiolini from US Synthetic, and Alaska Energy Authority Igiugig Village Council, U.S. Department of Energy Office of Energy Efficiency \& Renewable Energy and Office of Indian Energy Policy \& Programs. The Authors of Section 8 acknowledge Hydrodata \& Maira staff for all support given, and want to say that in September 2018 Iren Energia SpA finalized the acquisition of the controlling interest in Maira S.p.A., taking over the share of Hydrodata SpA. The technical documentation and photographic shots within Section 9 were kindly granted by AISE Engineering and by Parenti Costruzioni. We thank ICOP, PATO and Injectsond companies for the assistance and documentation provided in the estimate phase. Special thanks to PERI for the technical study of the mobile formwork described here. A special thanks to the structural engineer Stefano Paterlini. The authors of Section 10 would like to thank Primiero Energia S.p.A. to share the hydropower plant data. Authors of Section 13 would like to acknowledge Hydrowatt S.p.A. and T.i.S-Nuoval S.p.A. for the support and data provided. Authors of Section 14 team thanks the board of IRPHE lab for their help in the project and in the settings of experiments in Herode Channel. The turbine rehabilitation project described in Section 15 has been made possible by the combined efforts of the entire Turbulent NV team and the Bali Greenschool technical team. Special thanks are due to the project engineers Cristobal Venegas and Wim Verheirstraeten (Turbulent NV) and Tony Vovers and Dita Anggraini (Bali Greenschool team) for their role in the implementation, commissioning and maintenance of the project.

Conflicts of Interest: The authors declare no conflict of interest. The information and views set out in this article are those of the authors and do not necessarily reflect the official opinion of the European Commission.

Disclaimer: This paper does not reflect the views of the European Commission or its services. It is a collection of case studies, organized by the first author in his own capacity independently from the work and activity carried out at the European Commission. The authors of each section claim that results reported have not been published elsewhere; when part of results are already published elsewhere, proper references are provided, considering that 
most of the results and pictures are owned by the authors, being the case studies developed by the companies of the authors.

\section{Acronyms}

\begin{tabular}{ll} 
RDF12C & speed governor \\
RDT14C & voltage governor \\
FCR & frequency containment reserve \\
FRR & frequency restoration reserve \\
HydEA & Hydro Efficiency Analysis \\
KOOS & Kaplan Online Optimization System \\
O\&M & Operation and Maintenance \\
$Q$ & flow rate (m $\left.{ }^{3} / \mathrm{s}, 1 / \mathrm{s}\right)$ \\
$H$ & head (m) \\
$P$ & power (kW, MW) \\
$N$ & rotational speed (rpm) \\
WUA & weighted usable area \\
USACE & U.S. Army Corps of Engineers \\
BPA & Bonneville Power Administration \\
ORPC & Ocean Renewable Power Company \\
PCD & polycrystalline diamond \\
DN & nominal diameter (mm, m) \\
PMG & Permanent Magnet Generator \\
$Z$ & elevation (m) \\
$E$ & energy (GWh) \\
$C$ & cost ( $€$ ) \\
PRV & pressure reducing valve \\
PRS & Pressure Reduction Stations \\
ERH & Energy Recovery Hydropower \\
SHPP & Small Hydro Power Plant \\
FDC & Flow Duration Curve \\
ESD & Emergency Shut Down \\
HPM & Hydrostatic Pressure Machine \\
CFD & Computational Fluid Dynamics \\
$u$ & runner tangential speed (m/s) \\
$v$ & \\
& flow speed (m/s) \\
\hline &
\end{tabular}

\section{References}

1. International Hydropower Association. 2020 Hydropower Status Report Sector Trends and Insights; IHA Central Office: London, UK, 2020.

2. Branche, E. The multipurpose water uses of hydropower reservoir: The SHARE concept. C. R. Phys. 2017, 18, 469-478. [CrossRef]

3. Frey, G.W.; Linke, D.M. Hydropower as a renewable and sustainable energy resource meeting global energy challenges in a reasonable way. Energy Policy 2002, 30, 1261-1265. [CrossRef]

4. Moran, E.F.; Lopez, M.C.; Moore, N.; Müller, N.; Hyndman, D.W. Sustainable hydropower in the 21st century. Proc. Natl. Acad. Sci. USA 2018, 115, 11891-11898. [CrossRef] [PubMed]

5. Acreman, M.C.; Ferguson, A.J.D. Environmental flows and the European water framework directive. Freshw. Biol. 2010, 55, 32-48. [CrossRef]

6. European Commission. RES Directive 2009/28/EC of the European Parliament and of the Council of 23 April 2009 on the promotion of the use of energy from renewable. Off. J. Eur. Union 2009, 5, 16-62.

7. Kougias, I.; Aggidis, G.; Avellan, F.; Deniz, S.; Lundin, U.; Moro, A.; Muntean, S.; Novara, D.; Pérez-Díaz, J.I.; Quaranta, E.; et al. Analysis of emerging technologies in the hydropower sector. Renew. Sustain. Energy Rev. 2019, 113, 109257. [CrossRef] 
8. Trivedi, C.; Agnalt, E.; Dahlhaug, O.G. Investigations of unsteady pressure loading in a Francis turbine during variable-speed operation. Renew. Energy 2017, 113, 397-410. [CrossRef]

9. Jain, S.V.; Patel, R.N. Investigations on pump running in turbine mode: A review of the state-of-the-art. Renew. Sustain. Energy Rev. 2014, 30, 841-868. [CrossRef]

10. Bozhinova, S.; Hecht, V.; Kisliakov, D.; Müller, G.; Schneider, S. Hydropower converters with head differences below $2 \cdot 5$ m. Proc. Inst. Civ. Eng. Energy 2013, 166, 107-119. [CrossRef]

11. Quaranta, E.; Revelli, R. Gravity water wheels as a micro hydropower energy source: A review based on historic data, design methods, efficiencies and modern optimizations. Renew. Sustain. Energy Rev. 2018, 97, 414-427. [CrossRef]

12. Hogan, T.W.; Cada, G.F.; Amaral, S.V. The status of environmentally enhanced hydropower turbines. Fisheries 2014, 39, 164-172. [CrossRef]

13. Sari, M.A.; Badruzzaman, M.; Cherchi, C.; Swindle, M.; Ajami, N.; Jacangelo, J.G. Recent innovations and trends in in-conduit hydropower technologies and their applications in water distribution systems. J. Environ. Manag. 2018, 228, 416-428. [CrossRef] [PubMed]

14. Wang, C. A Guide for Local Benefit Sharing in Hydropower Projects; Social Development Working Papers; The World Bank Social Development: Washington, DC, USA, 2012; Volume 128.

15. International Commission for the Protection of the Danube River (ICPDR). Hydropower Case Studies and Good Practice Examples, ANNEX to "Guiding Principles on Sustainable Hydropower Development in the Danube Basin"; Vienna International Centre: Vienna, Austria, 2013.

16. International Hydropower Association. Better Hydro: Compendium of Case Studies 2017; IHA Central Office: London, UK, 2017.

17. Eker, I. Governors for hydro-turbine speed control in power generation: A SIMO robust design approach. Energy Convers. Manag. 2004, 45, 2207-2221. [CrossRef]

18. Codice di Rete Italiano. Available online: https://www.terna.it/it/sistema-elettrico/codici-rete/codice-reteitaliano (accessed on 20 October 2020).

19. Pauletta, G. Sistema di regolazione elettroidraulico Hydroart tipo PR80 per turbine idrauliche-ENEL-1993. Internal Document. (In Italian)

20. Sannolo, N.; Bonjean, M.; Cuvato, D.; Pasquettaz, R.; Caretti, P. Innovate to Optimise; International Water Power Dams Construction: Dartford, UK, 2019; pp. 28-31.

21. CECT. Recommendations for the Design, Manufacture and Erection of Steel Penstocks of Welded Construction for Hydroelectric Installations; European Committee of Boiler, Vessel and Pipe Work Manufacturers: Paris, France, 1980.

22. Koutnik, J.; Varner, M.; Linhart, V. Service Life Determination of a 50 Years Old Twin Penstock PSPP Stechovice Internationales Seminar Wasserkraftanlagen; TU Wien: Vienna, Austria, 2000.

23. Duparchy, F.; Chambas, P.A.; Marin, H.; Pereira, F.; Combaz, T. Impact of pressure fluctuation on the fatigue life of high pressure penstocks. In Proceedings of the Conference on High Strength Steels for Hydropower Plants; Graz University of Technology: Graz, Austria, 2013.

24. Nicolet, C.; Bollaert, E. Hydroelectric Power Plant Real-time Monitoring System and Method, 7.5. European Patent EP 2,801,879 B1, 27 September 2017.

25. Nicolet, C.; Béguin, A.; Bollaert, E.; Boulicaut, B.; Gros, G. Real-time simulation monitoring system for hydro plant transient surveys. Int. J. Hydropower Dams 2015, 22, 62-69.

26. Nicolet, C.; Greiveldinger, B.; Hérou, J.-J.; Kawkabani, B.; Allenbach, P.; Simond, J.-J.; Avellan, F. High Order Modeling of Hydraulic Power Plant in Islanded Power Network. IEEE Trans. Power Syst. 2007, 22, 1870-1881. [CrossRef]

27. Nicolet, C.; Dreyer, M.; Beguin, A.; Bollaert, E.; Torrent, S.; Dayer, J.-D. Hydraulic Transient Survey at Cleuson-Dixence with Real-Time Hydro-Clone Monitoring System. In Proceedings of the HYDRO 2018 Conference, Gdansk, Poland, 15-17 October 2018.

28. Frequency-Containment-Reserve. Available online: https://www.emissions-euets.com/internal-electricitymarket-glossary/793-frequency-containment-reserve (accessed on 20 October 2020).

29. Hachem, F.E.; Schleiss, A.J. A review of wave celerity in frictionless and axisymmetrical steel-lined pressure tunnels. J. Fluids Struct. 2011, 27, 311-328. [CrossRef]

30. Pachoud, A.J. Influence of Geometrical Imperfections and Flaws at Welds of Steel Liners on Fatigue Behavior of Pressure Tunnels and Shafts in Anisotropic Rock. Ph.D. Thesis, EPFL, Lausanne, Switzerland, 2017. 
31. E08 Committee. Practices for Cycle Counting in Fatigue Analysis; ASTM International: West Conshohocken, PA, USA, 2017.

32. Niesłony, A. Determination of fragments of multiaxial service loading strongly influencing the fatigue of machine components. Mech. Syst. Signal Process. 2009, 23, 2712-2721. [CrossRef]

33. British Standard. BS 79102013 Guide to Methods for Assessing the Acceptability of Flaws in Metallic Structures; British Standard: London UK, 2013; ISBN 0-580-45965-9.

34. Dreyer, M.; Nicolet, C.; Gaspoz, A.; Biner, D.; Rey-Mermet, S.; Saillen, C.; Boulicaut, B. Digital Clone for Penstock Fatigue Monitoring. In Proceedings of the 8th IAHR International Meeting of the Workgroup on Cavitation and Dynamic Problems in Hydraulic Machinery and Systems, Stuttgart, Germany, 9-11 October 2019.

35. Cazzaniga, R.; Rosa-Clot, M.; Rosa-Clot, P.; Tina, G.M. Integration of PV floating with hydroelectric power plants. Heliyon 2019, 5, e01918. [CrossRef]

36. Bruder, A.; Tonolla, D.; Schweizer, S.P.; Vollenweider, S.; Langhans, S.D.; Wüest, A. A conceptual framework for hydropeaking mitigation. Sci. Total Environ. 2016, 568, 1204-1212. [CrossRef]

37. Heller, P.; Bollaert, E.; Schleiss, A.J. Optimal Reservoir Use of a Multipurpose Run-of-River Powerplant for Hydropeaking Mitigation. In Proceedings of the Hydro2005: Policy into Practice, Villach, Austria, 17-20 October 2005; pp. 1-8.

38. Bovee, K. A Guide to Stream Habitat Analysis Using the Instream Flow Incremental Methodology; IFIP No. 12. No. 82/26; U.S. Fish and Wildlife Service: Washington, DC, USA, 1982.

39. Baumann, P.; Kirchhofer, A.; Schalchli, U. Risanamento Deflussi Discontinui-Pianificazione Strategica. In Un Modulo Dell'aiuto All'esecuzione Rinaturazione Delle Acque; Ufficio federale dell'ambiente: Bern, Switzerland, 2012.

40. Allen, L.; Conder, T.; Annear, C. Test of Weighted Usable Area Estimates Derived from a Phabsim Model for Instream Flow Studies on Trout Streams. N. Am. J. Fish. Manag. 1987, 7, 339-350.

41. Trumbo, B.A.; Ahmann, M.L.; Renholds, J.F.; Brown, R.S.; Colotelo, A.H.; Deng, Z.D. Improving hydroturbine pressures to enhance salmon passage survival and recovery. Rev. Fish Biol. Fish. 2014, 24, 955-965. [CrossRef]

42. Normandeau Associates Inc.; Skalski, J.R.; Townsend, R.L. Turbine Operational Effects on Survival/Condition of Yearling Chinook Salmon at Ice Harbor Dam. March 2007, unpublished.

43. Martinez, J.J.; Deng, Z.D.; Titzler, P.S.; Duncan, J.P.; Lu, J.; Mueller, R.P.; Tian, C.; Trumbo, B.A.; Ahmann, M.L.; Renholds, J.F. Hydraulic and biological characterization of a large Kaplan turbine. Renew. Energy 2019, 131, 240-249. [CrossRef]

44. Johnson, P.N.; Nealson, P.; Steig, T.; Sullivan, C.; Miller, J.; Haffey, S.; Gray, M.; Geris, K.; Hitchko, K.; Skalski, J.; et al. Characterization of juvenile salmonid vertical distribution in unit 1, Ice Harbor Dam. 2015; unpublished.

45. Malerba, C. L'acqua, il Futuro. In Ispirazione Sociologica e Gestione Dell'acqua, Risorsa Primaria; arabAFenice: Cuneo, Italy, 2012.

46. Giddens, A. The Consequences of Modernity; Polity Press: Cambridge, UK, 1991.

47. Bromaghin, A.; Ali, M.; Ravens, T.; Petersen, T.; Hoffman, J. Experimental Study of Abrasion Characteristics for Critical Sliding Components for Use in Hydrokinetic Devices. Renew. Energy 2014, 66, 205-214. [CrossRef]

48. Tyler, R. Commercial Systems at ORPC, personal interview. 17 June 2020.

49. Khonsar, M.; Booser, E. Applied Tribology: Bearing Design and Lubrication, 3rd ed.; John Wiley \& Sons, Ltd.: Hoboken, NJ, USA, 2001; pp. 153-154.

50. Marnagh, C. Using Tidal Energy to Power Off-the-Grid Towns. NBC News, Mach. 3 April 2019. Available online: https://www.nbcnews.com/mach/video/using-tidal-energy-to-power-off-the-grid-towns1471394883522 (accessed on 20 October 2020).

51. Inoue, K.; Deguchi, K.; Okude, K.; Fujimoto, R. Development of the Water-Lubricated Thrust Bearing of the Hydraulic Turbine Generator. In IOP Conference Series: Earth and Environmental Science; IOP Publishing: Bristol, UK, 2012; Volume 15, p. 072022.

52. Massarutto, A. Agriculture, Water Resources and Water Policies in Italy. Feem Work. Pap. 2000, 33-99.

53. Fellini, S.; Vesipa, R.; Boano, F.; Ridolfi, L. Multipurpose Design of the Flow-Control System of a Steep Water Main. J. Water Resour. Plan. Manag. 2018, 144, 05017018. [CrossRef]

54. Rani, D.; Moreira, M. Simulation-optimization modeling: A survey and potential application in reservoir systems operations. Water Resour. Manag. 2010, 24, 1107-1138. [CrossRef] 
55. Sinagra, M.; Aricò, C.; Tucciarelli, T.; Amato, P.; Fiorino, M. Coupled Electric and Hydraulic Control of a PRS Turbine in a Real Transport Water Network. Water 2019, 11, 1194. [CrossRef]

56. Viccione, G.; Amato, R.; Martucciello, M. Hydropower Potential from the AUSINO Drinking Water System. Proceedings 2018, 2, 688. [CrossRef]

57. Van Dijk, M.; Van Vuuren, S.J.; Cavazzini, G.; Santolin, A.; Niebuhr, C.M. Optimization of Cascading Conduit Hydropower Plants in a Water Distribution System. In Proceedings of the Hydro Conference, Porto, Portugal, 14-16 October 2019; Aqua-Media International Ltd.: Wallington, Surrey, 2019.

58. White, J. Recovering energy from an existing conduit. Int. Water Power Dam Constr. 2011, 3, 18-20.

59. Penninger, G.; Benigni, H. Numerical Simulation and Design of Spherical Valves for Modern Pump Storage Power Plants. In Proceedings of the 14th International Seminar on Hydropower Plants, Vienna, Austria, 22-24 November 2006.

60. Quaranta, E. Optimal Rotational Speed of Kaplan and Francis Turbines with Focus on Low-Head Hydropower Applications and Dataset Collection. J. Hydraul. Eng. 2019, 145, 04019043. [CrossRef]

61. European Commission. HYLOW Project: Development of Hydropower Converters with Very Low Head Differences. European Project Number 212423 Funded under the Framework Program 7-Energy 2007 (FP7-Energy-2007-1-RTD), 2008-2013. Available online: http://www.hylow.eu/ (accessed on 20 October 2020).

62. Paish, O. Small hydropower: Technology and current status. Renew. Sustain. Energy Rev. 2002, 6, 537-556. [CrossRef]

63. Senior, J.; Saenger, N.; Müller, G. New hydropower converters for very low-head differences. J. Hydraul. Res. 2010, 48, 703-714. [CrossRef]

64. Senior, J.A. Hydrostatic Pressure Converters for the Exploitation of very Low Head Hydropower Potential. Ph.D. Thesis, University of Southampton, Southampton, UK, 2009.

65. Linton, N.P. Field Trials and Development of A Hydrostatic Pressure Machine. Ph.D. Thesis, University of Southampton, Southampton, UK, 2013.

66. Paudel, S. Experimental and Numerical Study of Dethridge Wheel for Pico-scale Hydropower Generation. Ph.D. Thesis, Darmstadt University, Darmstadt, Germany, 2015.

67. Butera, I.; Fontan, S.; Poggi, D.; Quaranta, E.; Revelli, R. Experimental Analysis of Effect of Canal Geometry and Water Levels on Rotary Hydrostatic Pressure Machine. J. Hydraul. Eng. 2019, 146, 04019071. [CrossRef]

68. Quaranta, E. The Revival of Old Hydraulic Turbines for Innovative Hydropower Generation: Water Wheels, Archimedes Screws, Deriaz and Girard Turbines. Curr. Trends Civ. Struct. Eng. 2020, 5, 1-4.

69. Audrius, Z.; Aggidis, G. State of the art in numerical modelling of Pelton turbines. Renew. Sustain. Energy Rev. 2015, 45, 135-144.

70. Müller, G.; Kauppert, K. Performance characteristics of water wheels. J. Hydraul. Res. 2004, 42, 451-460. [CrossRef]

71. Quaranta, E.; Müller, G. Sagebien and Zuppinger water wheels for very low head hydropower applications. J. Hydraul. Res. 2018, 56, 526-536. [CrossRef]

72. Metzler, M.; Pelz, P.F. Draft tube design method for operation at optimal coefficient of performance in low head hydropower. Wasserwirtschaft 2015, 105, 13-17. [CrossRef]

73. Amaral, S.V.; Hecker, G.E.; Dixon, D.A. Designing Leading Edges of Turbine Blades to Increase Fish Survival from Blade Strike. In EPRI-DOE, Conference on Environmentally-Enhanced Hydropower Turbines; EPRI Report, No. 1024609; EPRI: California, CA, USA, 2011.

74. Cada, G.F.; Coutant, C.C.; Whitney, R.R. Development of Biological Criteria for the Design of Advanced Hydropower Turbines; No. DOE/ID-100578; EERE Publication and Product Library: Washington, DC, USA, 1997.

75. Cook, T.C.; Hecker, G.E.; Amaral, S.V.; Stacy, P.S.; Lin, F.; Taft, E.P. Final Report-Pilot Scale Tests Alden/Concepts NREC Turbine; US Department of Energy: Washington, DC, USA, 2003.

76. Abernethy, C.S.; Amidan, B.G.; Cada, G.F. Simulated Passage through A Modified Kaplan Turbine Pressure Regime: A Supplement to Laboratory Studies of the Effects of Pressure and Dissolved Gas Supersaturation on Turbine-Passed Fish; No. PNNL-13470-A; Pacific Northwest National Lab. (PNNL): Richland, WA, USA, 2002.

77. Sungai Ayung. Available online: https://id.wikipedia.org/wiki/Sungai_Ayung (accessed on 22 June 2020).

78. Richmond, M.C.; Serkowski, J.A.; Ebner, L.L.; Sick, M.; Brown, R.S.; Carlson, T.J. Quantifying barotrauma risk to juvenile fish during hydro-turbine passage. Fish. Res. 2014, 154, 152-164. [CrossRef]

79. Quaranta, E.; Riasi, A.; Revelli, R. The Very Low Head Turbine for hydropower applications: A review. Renew. Sustain. Energy Rev. 2020. submitted. 
80. Fraser, R.; Deschênes, C.; Kueny, J.L.; O’Neil, C.; Leclerc, M. VLH: Development of a new turbine for Very Low Head sites. In Waterpower XV; Hydro Review: Chattanooga, TN, USA, 2007.

81. Lautier, P.; Ndjana, H.J.N.; Leclerc, M.; O'Neil, C. Improved operation and power production of a Very Low Head hydraulic turbine. In Waterpower XV; Hydro Review: Chattanooga, TN, USA, 2007; Available online: http://www.vlh-turbine.com/DE/PDF/evenements/WaterPower2007_Philippe_Lautier.pdf (accessed on 20 October 2020).

82. Leclerc, M. The very low head turbo generator: A new turbine for profitable harnessing of very low head applications. In Hydro 2007; Aqua Media International: Granada, Spain, 2007.

83. Arquilla, M.; Pasut, F. A Novel MPPT controller for Variable Speed hydro generators. In Proceedings of the Vienna hydro International Conference, Vienna, Austria, 26-28 November 2014; pp. 569-575.

84. Égré, D.; Senécal, P. Social impact assessments of large dams throughout the world: Lessons learned over two decades. Impact Assess. Proj. Apprais. 2003, 21, 215-224. [CrossRef]

85. Quaranta, E.; Wolter, C. Sustainability assessment of hydropower water wheels with downstream migrating fish. Sustain. Energy Technol. Assess.. submitted.

86. Gielen, D. Renewable energy technologies: Cost analysis series. Hydropower 2012, 1, IRENA.

Publisher's Note: MDPI stays neutral with regard to jurisdictional claims in published maps and institutional affiliations.

(C) 2020 by the authors. Licensee MDPI, Basel, Switzerland. This article is an open access article distributed under the terms and conditions of the Creative Commons Attribution (CC BY) license (http://creativecommons.org/licenses/by/4.0/). 